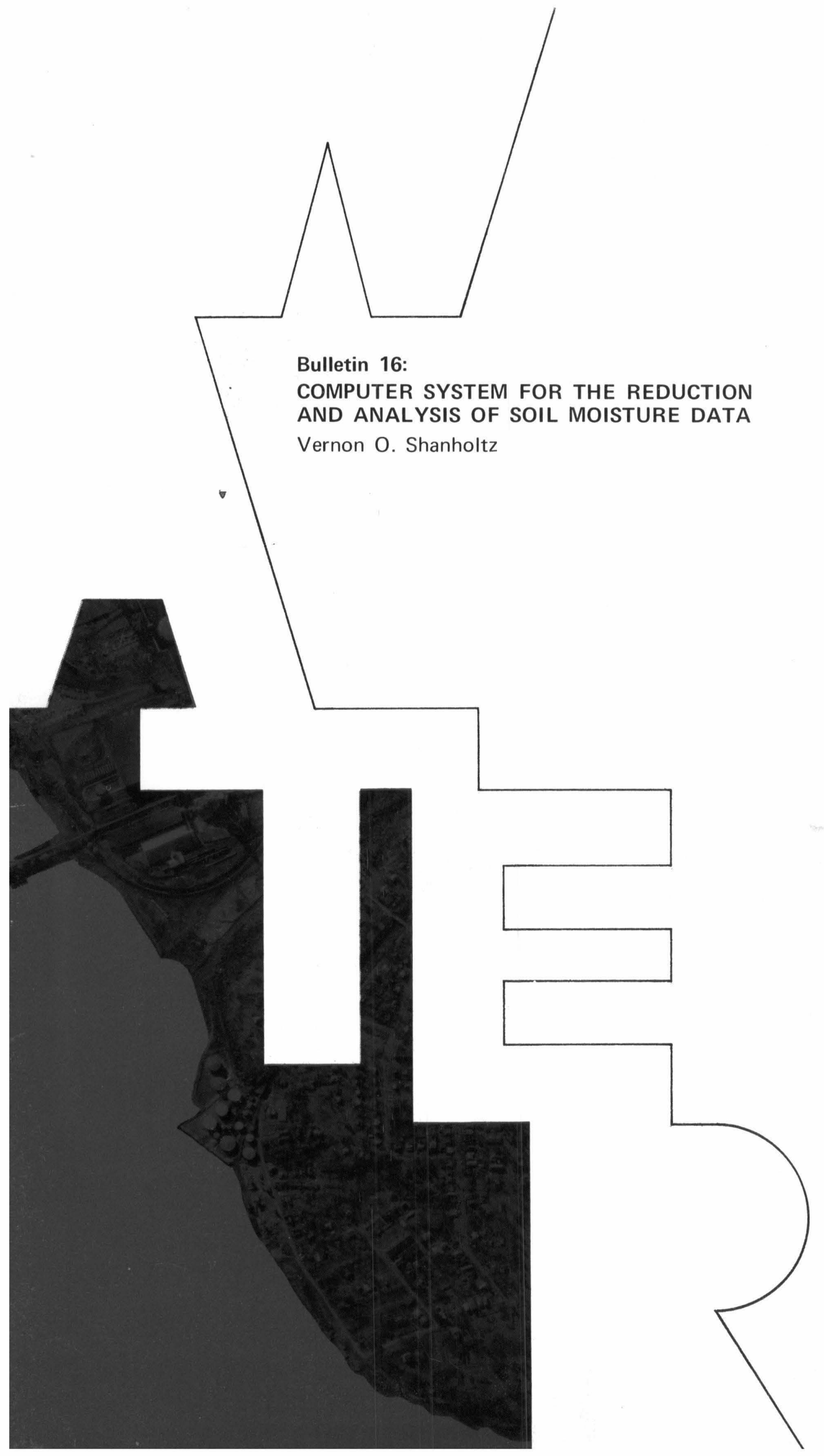




\title{
COMPUTER SYSTEM FOR THE REDUCTION AND ANALYSIS OF SOIL MOISTURE DATA
}

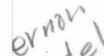 \\ V. O. Shanholtz \\ Research Instructor \\ Department of Agricultural Engineering
}

The work upon which this report is based was supported in part by funds provided by the United States Department of the Interior, Office of Water Resources Research, as authorized under the Water Resources Act of 1964 and in part by the V.P.I. Division of Research.

\author{
Water Resources Research Center \\ Virginia Polytechinc Institute \\ Blacksburg, Virginia \\ December 1968
}




$$
\begin{aligned}
& T D 201 \\
& V 57 \\
& \text { n. } 16 \\
& c .2
\end{aligned}
$$




\section{PREFACE}

Quantitative evaluation of soil moisture for extended periods of time is a basic requirement in many areas of research of agricultural engineering and the life sciences. Soil-water-plant relationships almost always require detailed soil moisture measurements. This can involve the handling, processing, and analysis of thousands of individual measurements.

This report presents a program written in Forton IV to facilitate the reduction and analysis of soil moisture data. The system was prepared in three sections to allow complete flexibility in handling, processing, and analyzing moisture data.

The system provides for the processing of basic field data; the computing and accumulating of the total and/or available soil moisture for selected depth intervals; and the plotting and coding of the above data for subsequent evaluation by analysis of variance techniques.

The program allows for a detailed and comprehensive study of a wide range of statistically designed experiments to evaluate the availability and quantity of soil moisture. Probably the greatest utility of the system is its ability to provide a complete review of the moisture status at all levels in the soil profile and then to quickly ascertain all possible treatment combinations and interactions within the experimental design.

William R. Walker

Director 



\section{TABLE OF CONTENTS}

INTRODUCTION . . . . . . . . . . . . . . 1

SOIL MOISTURE REDUCTION ............. . 2

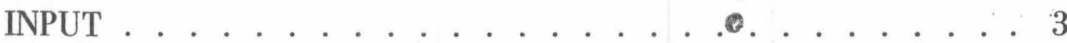

Header Card . . . . . . . . . . . . . . . 3

Soil Moisture Tension Table ............ 3

Identification Tables ............... . . 4

Block - Treatment Table ... . . . . . . . 5

Access Tube Identification . . . . . . . . . . . 7

Depth Identification Table ........... 7

Parameter Cards . . . . . . . . . . . . . 8

Basic Data . . . . . . . . . . . . 14

PROGRAM CONSTANTS .............. 15

DATA PREPARATION ................ . . 15

Basic Input Data . . . . . . . . . . . . 15

Identification Tables ............ . 20

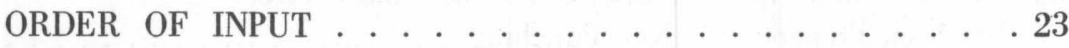

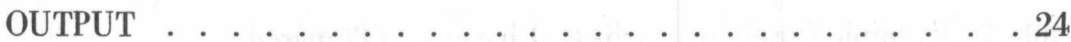

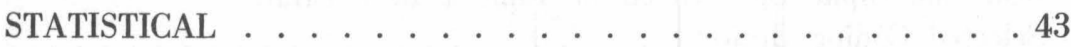

APPENDIX A ................... 44

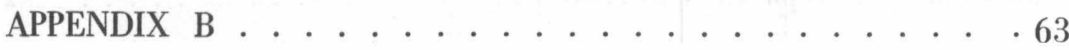




\section{LIST OF FIGURES}

Figure 1. Schematic Diagram of a Typical Experimental Plot Design of Two Treatments - Conventional Tillage and No Tillage - Blocked 4 Times . . . . . . . . 6

\section{LIST OF TABLES}

Table 1. Code to Identify the Depth at which Moisture

Data is being Plotted ................. 13

Table 2. Abbreviated Display of Data Resulting from Section 1 to be used to Illustrate Data Preparation and Results for Section $2 \ldots \ldots \ldots \ldots$

Table 3. Identifications and Corresponding Coding for Various Block - Treatment Combinations, Depths, and Access Tubes for No-Tillage Experiment Located at Blacksburg, Virginia . . . . . . . . . . . . . . . 19

Table 4. The Identification Data Given in Table 3 after

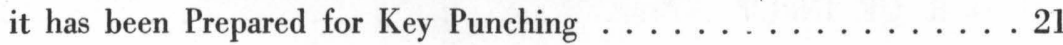

Table 5. Example Parameter Cards that have been Prepared from the Input Data Given in Table 2 to Illustrate

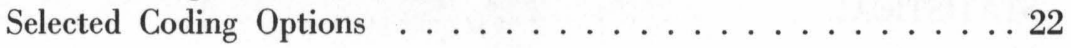

Table 6. Identification Dictionary that has been Stored in the Computer Preparatory to Processing . . . . . . . . . 25

Table 7. Example Output for Identifications that were not Present in Dictionary . . . . . . . . . . . . . . . 25

Table 8. Abbreviated Listing of Punch Cards Containing Coded Soil Moisture Data for Subsequent Analysis of Variance

Table 9. Abbreviated Listing of the Data Given in Table 8 after Being Sorted for Analysis of Variance for a Completely Cross - Classified Statistical Design...........27 
Table 10. Abbreviated Listing of Optional Output when Punch Cards are Not Required for Analysis of Variance. . . . . . . . . . . . . . . . . . . . . . . . . 29

Table 11. Total Accumulated Soil Moisture Summary for the Input Date Given in Table 2. . . . . . . . . 32

Table 12. Total Soil Moisture at 6" Increments for the Input Data Given in Table 2. . . . . . . . . . . . 33

Table 13. Total Soil Moisture Summary at 6" Increments for the Average of Variance Block - Treatment Combination of the Input Data Given in Table $2 \ldots \ldots \ldots \ldots . .35$

Table 14. Example Plottings of the Data Given in Table 11 for 2 Combinations (Block 1 - Treatments 1 and 2 and Block 2 - Treatments 1 and 2) at Depths $0^{\prime \prime}-3^{\prime \prime}$,

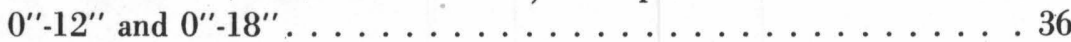

Table 15. Example Plottings of the Data Given in Table 12 for 2 Combinations (Block 1 - Treatments 1 and 2 and Block 2 - Treaments 1 and 2) at Depths 0" $0^{\prime \prime}-6^{\prime \prime}$, $6 "-12^{\prime \prime}$ and $12 "-18^{\prime \prime} \ldots \ldots \ldots \ldots \ldots \ldots \ldots \ldots \ldots$

Table 16. Example Plottings of the Data Given in Table 13 for 3 Groups at 3 Depths $\left(0^{\prime \prime}-6^{\prime \prime} .66^{\prime \prime}-12^{\prime \prime}\right.$ and

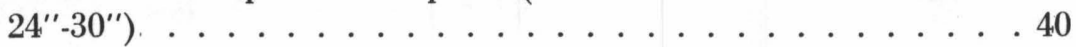

Table 17. Example of 4 Curves Plotted on the same Graph for Comparison. See Table 18 for Symbol Identification. . . . . . . . . . . . . . . . . 42

Table 18. Characters used to Identify Coordinate Points. . . . . . . . . . . . . . . . 44 



\section{INTRODUCTION}

The quantitative evaluation of soil moisture through extended periods is a basic requirement in areas of research related to engineering, agriculture, and the life sciences. Invariably, it involves the processing and analysis of thousands of measurements. These are most often taken with nuclear soil moisture monitoring equipment.

It is the purpose of this report to present a system of computer programs, written in Fortran IV for the IBM 7040/1401 system, but readily convertible to other systems, designed specifically to expedite the reduction and analysis of soil moisture data. The system contains 3 unique and independent programs or sections. The first section consists of a reduction of the basic field data, the second section is a coding, summary and plotting routine, and the third section is an analysis of variance routine. These separations allow for a more general approach in the reduction procedure. Data are collected in the field, key punched from the field sheets, and processed in exactly the same order as they are obtained.

All data are properly identified so that no partiuclar input or processing order is necessary. The only requirement is that groups of data must be kept independent of each other. Following this procedure, all data and field notes can be processed (see Appendix Al) regardless of whether or not they are complete. Incomplete records will result when weather conditions or instrument malfunction prohibit the collection of moisture data over the entire experimental design. The second section of the system was designed so that all or only isolated segments of the output from Section 1 could be selected as the basic input data.

In most analysis of variance procedures, the order of input of the data is very important. Consequently, the soil moisture values to be used for such analyses must be placed in the required order. A function of Section 2 is to code the basic input data from Section 1 in such a way that it can be rapidly placed in the correct order by a minimum of passes through a sorter. For example, if the breakdown for analysis of variance includes blocks, dates, depths, and treatments, each moisture reading must be coded and placed on punch cards so that each of these items can be placed in its relative position for analysis by the sorter (see Appendix A4).

The basic features of the system may be summarized as follows:

a. Basic field data are processed and stored on magnetic tape as percentage moisture by volume (see Appendix A1). 
b. Accumulated total or available soil moisture values can be determined by 3 " increments to a depth of 30 " and then by 6 " increments to a maximum depth of 72 ".

c. Total or available soil moisture values can be determined at 6" increments to a maximum depth of 72 ".

d. Individual or groups of treatments can be summed over the entire experiment to determine the average total or available soil moisture for the increments given in item $b$ above.

e. The data can be coded and placed on punch cards for subsequent analysis of variance or any other statistical analysis that may be desired.

f. The soil moisture values at any level can be plotted for any blocktreatment combination evolving from items $\mathrm{b}$, $\mathrm{c}$, or $\mathrm{d}$. Instructions for plotting these curves must be given in the order of b, $c$, or $d$. They cannot be intermixed.

g. An analysis of variance for a completely cross-classified design can run using as input the data obtained through item e.

As noted above computations can be performed with either total or available soil moisture. Available soil moisture is generally assumed to be water available for plant use and is considered by many researchers to be the water content between $1 / 3$ and 15 atmospheres of tension. Total moisture is considered to be the total water held in the soil profile as determined by readings from nuclear moisture monitoring equipment.

The remainder of this report will be concerned with the function of Section 2 of the system. The presentation refers only to total soil moisture, however, the discussions are equally applicable to available soil moisture. Limited information is given in Appendixes A1, A2, A3, and A4 along with reference details as to where and how documentation of these sections can be obtained.

\section{SOIL MOISTURE REDUCTION}

As previously mentioned, Section 2 of the soil moisture reduction system is primarily a coding, summary, and plotting routine. In the items listed above under program features, $b, c, d, e$, and $f$ may be attributed 
to Section 2. The performance of all items is optional, i.e. by proper coding of parameter cards various functions can be either performed or omitted.

A listing of the source program for Section 2 is given in Appendix B1 and a detailed discussion of the program variables is given in Appendix B2. Most input/output variables have been omitted since they are discussed at their appropriate locations in the report.

\section{INPUT}

The input for Section 2 consists of (a) header card, (b) moisture tension tables, (c) identification tables, (d) parameter cards, and (e) soil moisture data. Items $\mathrm{a}, \mathrm{b}, \mathrm{c}$, and $\mathrm{d}$ are on punch cards (these data are actually transferred to a system utility tape, SSU05, by an IBM 1401 processor preparatory to being processed by the IBM 7040 computer) and item e is on magnetic tape. Item e is a direct output from Section 1.

\section{Header Card}

The function of the header card is to specify the type of analysis to be performed. Computations are based on available soil moisture when TYPE = 1 and total soil moisture when TYPE $=2$.

The form of this card follows:

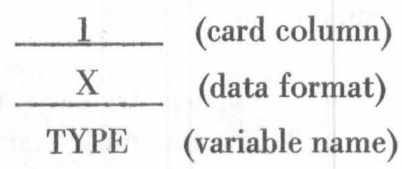

\section{Soil Moisture Tension Table}

The soil moisture tension table contains the maximum available moisture and the lower limit of the available range for each block-treatmentdepth combination in a given experiment. The available moisture is determined using this table and the percentage soil moisture values from Section 1. Note that this table is not required when total soil moisture is being determined, i.e. when TYPE $=2$.

A common practice is to consider the available soil moisture as the soil moisture content between $1 / 3$ and 15 atmospheres tension. With this criteria, the soil moisture tension table would contain percentage moisture 
by volume, as determined from the laboratory, for the 15 atmosphere point (lower limit) and the 1/3 atmosphere point (upper limit).

The data card form for this table is given below followed by a description of variables. The data in columns 7-11 and 12-16 must be right justified when decimal points are not punched.

\begin{tabular}{cccccc}
$1-2$ & $3-4$ & $5-6$ & $7-11$ & $12-16$ & $17-18$ \\
\hline XX & XX & XX & XX.XX & XX.XX & XX \\
\hline N3 & N2 & N1 & B & AIJL(N3,N2,N1) & NSTP
\end{tabular}

Symbol

Description of Variables

AIJL

Lower limit of available soil moisture range. This limit is usually considered to be the moisture content at 15 atmospheres tension.

B Upper limit of available soil moisture range. This limit is commonly considered to be the moisture content at 1/3 atmosphere tension.

N1 Integer code assigned to each depth at which soil moisture readings were obtained. This code must start with 1 and increase sequentially. A maximum of 12 values can be used.

N2 Integer code assigned to each treatment within the experiment. This code must start with 1 and increase sequentially. A maximum of 12 treatments can be used.

N3 Integer code assigned to the blocks within the experiment. The code must start with 1 and increase sequen: tially. A maximum of 12 blocks can be used.

NSTP

Index code indicating that the last card in the soil moisture tension table has been loaded.

Identification Tables

The system was designed so that meaningful alphabetic or numeric identification can be assigned to blocks, treatments, or access tubes. For statistical analyses where order is important, the data must be coded so 
that they can be rapidly placed in any desired sequence. The identification tables contain the identifications that were arbitrarily assigned at the initiation of the experiment to each block, treatment, and moisture access tube. They also contain an integer code for each respective block, treatment, and tube (e.g. if there are 2 treatments, treatment 1 would be designated as 1 and treatment 2 as 2). The integer codes are arbitrarily assigned and must begin with 1 and increase sequentially.

For this system, 3 tables are required to obtain proper coding.
a. Block-treatment table
b. Access tube identification table
c. Depth identification table

Block-Treatment Table -- In most experimental plot designs that are replicated or blocked, the block-treatment identification is combined into one symbol, e.g. the treatment could be represented as NTM (no-tillage moisture), the block or replication as 1, all combined as NTM1. A typical experimental layout is shown in Figure 1. The integer codes associated with each block-treatment identification are shown in the format and variable description given below.

\begin{tabular}{cccc}
$1-6$ & $7-8$ & $9-10$ & 80 \\
\hline XXXXXX & XX & XX & X \\
\hline TPFORM & BKCODE & TRCODE & KODE9
\end{tabular}

Symbol

Description of Variables

BKCODE ( I ) Block or replicate code (this code must be integer and $\mathrm{I}=1,50$ the coding must progress in sequential order beginning with 1).

KODE9 A 9-punch in column 80 that identifies the last card of the block-treatment table.

TPFORM ( I ) Block-treatment identification. This code may contain $\mathrm{I}=1,50$ either alphabetic or numeric information (e.g. NTM4 for no-tillage moisture-block 4).

TRCODE ( I ) Treatment code. (This code must be integer and the $\mathrm{I}=1,50$ coding must progress in sequential order beginning with 1). 


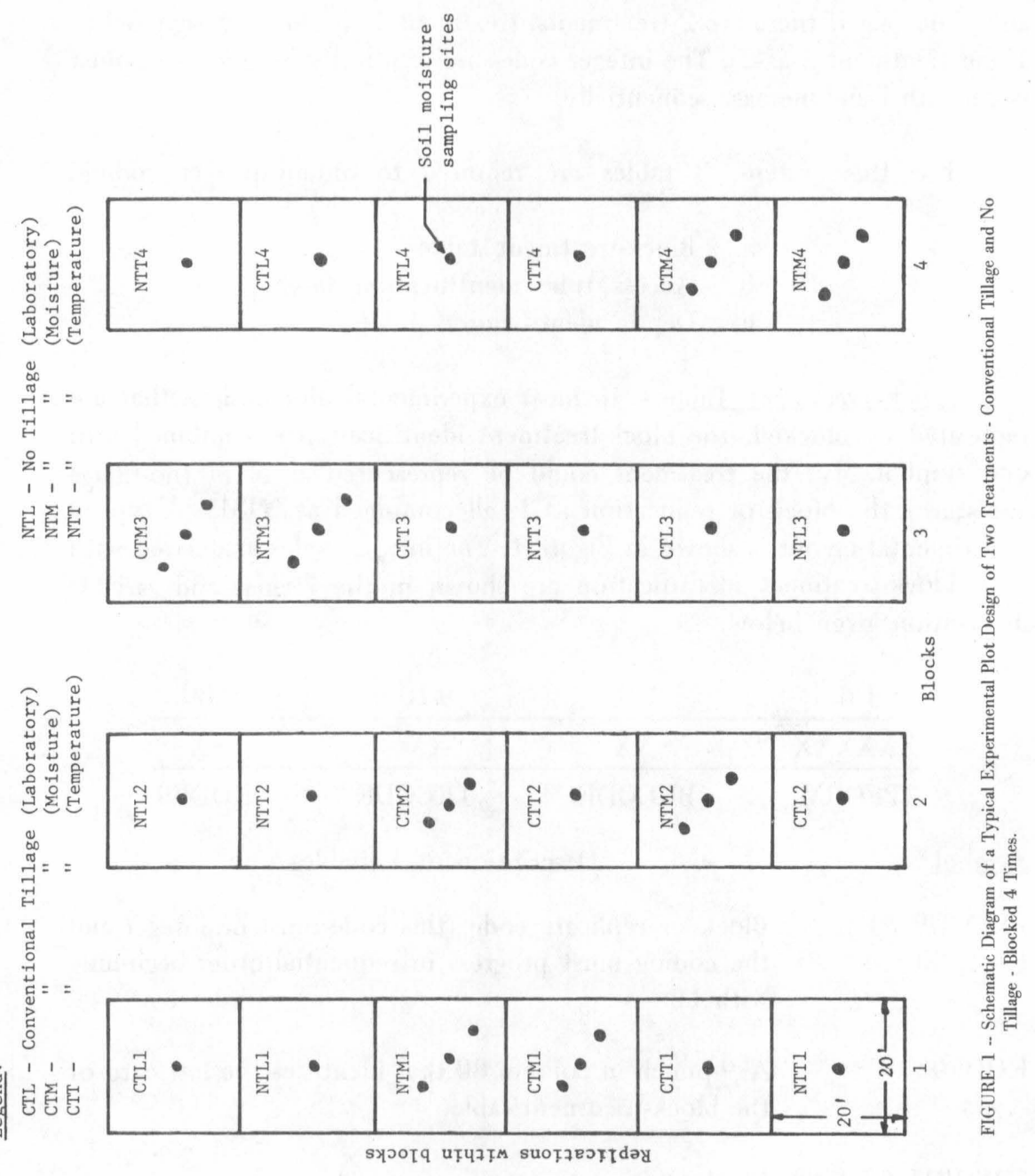


Access Tube Identification Table -- This table contains the identification assigned to all access tubes located within a given plot. Only those tubes within a given plot contain differing identifications (see Figure 1). For example, in a statistically designed experiment containing 2 treatments, blocked or replicated 4 times and 3 access tubes per plot, there would be a total of 24 access tubes; however, there should be only 3 differing tube identifications, e.g. A, B, and C or 1,2 , and 3 for the three within plot tubes. An integer code is placed in columns $4-5$ as shown in the following format specification and variable description.

\begin{tabular}{ccc}
$1-3$ & $4-5$ & 80 \\
\hline XXX & XX & $X$ \\
\hline TUBET & TUBCOD & KODE9
\end{tabular}

Symbol

Description of Variables

KODE9 A 9-punch in column 80 that identified the last card of the tube identification table.

TUBCOD ( I ) Soil moisture access tube code number (this code must $\mathrm{I}=1,50$ be integer and the coding must progress in sequential order beginning with 1 ).

TUBET (I) Tube identification. This code may contain either alpha$\mathrm{I}=1,50$ betic or numeric information.

Depth Identification Table -- This table contains the depths at which soil moisture readings were obtained and respective DEPCOD code values. Conversion factors for converting soil moisture from percentages by volume to inches of water are given for each DEPTHT value. The format for this table followed by a description of variables is given below. Indicated decimal points are not to be keypunched because they only represent where the decimal point will be placed when the data is processed by the computer.

\begin{tabular}{cccc}
$1-2$ & $3-4$ & $5-9$ & 80 \\
\hline XX. & XX & XXXXX. & X \\
\hline DEPTHT & DEPCOD & CONV & KODE9
\end{tabular}

Symbol

Description of Variables

CONV ( I ) Factor for converting percentage soil moisture for a $\mathrm{I}=1,15$ given depth to inches of water. 
DEPCOD ( I ) Depth identification code (this code must be integer $\mathrm{I}=1,15$ and the coding must progress sequentially beginning with 1).

DEPTH ( I ) Depths at which soil moisture readings have been ob$\mathrm{I}=1,15$ tained, e.g. 0 (surface), 12", 24", and 36".

\section{Parameter Cards}

Two groups of parameter cards are required for proper program execution. The first group specifies such items as the location of the input data on magnetic tape, provides options for including or deleting specific functions or data, and provides the dimensions of the experimental design. The format specification for this first group of parameter cards followed by a description of variables is given below:

\begin{tabular}{ccccccc}
$-1-3$ & $4-8$ & $9-11$ & $12-16$ & $17-18$ & $19-20$ & $21-22$ \\
\hline XXX & XXXXX XXX & XXXXX & XX & XX & XX \\
\hline BEG1 & BEG2 & END1 & END2 & KARDS & KUNIT6 & KUNIT1
\end{tabular}

\begin{tabular}{ccccccc}
$23-24$ & $25-26$ & $27-28$ & $29-30$ & $31-32$ & $33-34$ & $35-36$ \\
\hline $\mathrm{XX}$ & $\mathrm{XX}$ & $\mathrm{XX}$ & $\mathrm{XX}$ & $\mathrm{XX}$ & $\mathrm{XX}$ & $\mathrm{XX}$ \\
\hline NDUP & NBLKS NTRTS & NDPS & MAXDP1 & MAXDEP & NEND
\end{tabular}

\begin{tabular}{lll}
$37-38$ & $39-40$ & \\
\hline XX & XX1/ & XX \\
\hline NGX5 & TRCOMB & NERR
\end{tabular}

$1 /$ Continue with 2 digit fields until all data have been recorded for TRCOMB. Use additional data cards as required.

Symbol

Description of Variables

BEG1 Update code

BEG2 Sequence number. BEG1 and BEG2 identify the starting point a given analysis.

END1 Update code 
END2

KARDS

KUNIT1

KUNIT6

MAXDEP

MAXDP1

NBLKS
Data sequence number. END1 and END2 specify the end of a given analysis or group of data.

Index code with the following 2 options:

KARDS $=0$ : Cards are punched for subsequent analysis of variance (Section 3 )

KARDS $=1$ : Cards are not ' punched

Index code with the following 2 options:

KUNIT1 $=0$ : The soil moisture data will be coded, converted to inches of water and stored on S.SUO1. This variable must be set to 0 if plottings or data summaries by 3 " or 6 " increments are desired.

KUNIT1 $=1$ : The above data are not written on S.SUO1; therefore, no summaries or plottings will be made.

Index code with 2 possible options:

KUNIT6 $=0$ : The soil moisture data that have been coded for analysis of variance (punched cards) will be written on S.SUO6 for subsequent listing by a printer.

KUNIT6 $=1$ : The above option is by-passed.

The maximum depth for which soil moisture readings were obtained for all treatments in each block. The same number of replications is not required.

To perform an analysis of variance for a factorial design, the same number of replications must be taken on each block-treatment combination. For example, if 3 soil moisture tubes (replicates) have been placed in each treatment, then MAXDP1 is the maximum depth at which readings were made in all 3 tubes.

The total number of blocks in the experiment, e.g. if the overall experiment is blocked or replicated 4 times, NBLKS would equal 4. 
The total number of levels at which soil moisture readings were obtained, e.g. if readings were taken at the surface, 12", 18", and 24" NDPS would equal 4.

NDUP Normally, the surface moisture content is obtained at only one location within a given block-treatment combination (small plots only) and this reading is assumed to be representative for the entire treatment. As previously stated, analysis of variance computations with completely cross elassified models require the same number of observations in each block-treatment combination, e.g. if comparisons are to be made between treatments at the 0 (surface), 12", and 18" depths, moisture readings must be obtained at these 3 depths from all access tubes. For situations where soil moisture observations are being obtained from more than one access tube or location per treatment and one surface observation is assumed to be representative of the entire treatment area, the missing surface readings may be obtained by duplicating the surface reading taken. This can be accomplished by setting NDUP equal to the number of access tubes located in the treatment area.

NEND

Index code which determines the end of a given analysis. The following options are available.

NEND = 8: Proceed to the next group of input data. Normally, moisture readings taken on a given day constitute a group. In a normal analysis there will be a whole series of such groups.

NEND $=9$ : Last group of data. When KUNIT1 has been set equal to one, all processing is terminated. At this point all data have been coded and converted to inches of water for subsequent analysis of variance computations.

NEND $=9$ when KUNIT1 $=0$ : Processing continues with data summary and plotting options.

NEND = 99: Last group of data. No data summary of plotting options. 
NERR

NGX5

NTRTS

TRCOMB(NGX5) The treatments that are to be combined and averaged NGX5 $=1,75$

Index code with the following two options:

NERR $=0$ : Data encountered on the input tape which was not referenced in the identification tables are written on S.SUO8.

NERR $=1$ : The error output on S.SU08 is suppressed.

The NERR code is placed in the first two card columns following TRCOMB. For example, if TRCOMB ends in columns 59-60, place NERR in columns 61-62.

The number of groups to be read times 5. A maximum of 15 GROUPS or 75 TRCOMB values may be read from each parameter card.

The total number of treatments in each block.

to form GROUPS'

The second group of parameter cards supplies the various options that are available for data summary and plotting. The 3 data summaries are: (a) a listing of accumulated total soil moisture for specific units, (b) a listing of total soil moisture by 6" increments and (c) a listing of total soil moisture by $6 "$ increments for the treatment combinations designated by TRCOMB and averaged as GROUPS. Plottings can be made of all possible combinations of the above. The block-treatment coding previously assigned in the development of the identification tables determines the particular combination to be plotted. Four months of daily moisture readings can be plotted at 3 different depths on 1 sheet of 14-7/8 x 11 inch paper. A maximum of 4 curves, where each curve normally represents some particular treatment combination, can be plotted at each depth. Normally, better legibility results when 2 curves are plotted for comparison. The format specification for this group of parameter cards followed by a description of variables is given below.

\begin{tabular}{cccccccc}
$1-2$ & $3-4$ & $5-6$ & $7-8$ & $9-10$ & $11-12$ & $13-14$ & $15-16$ \\
\hline $\mathrm{XX}$ & $\mathrm{XX}$ & $\mathrm{XX}$ & $\mathrm{XX}$ & $\mathrm{XX}$ & $\mathrm{XX}$ & $\mathrm{XX}$ & $\mathrm{XX}$ \\
\hline
\end{tabular}

NBLOCK NTREAT NBL2 NTRT2 NBL3 NTRT3 NBL4 NTRT4 


\begin{tabular}{cccccc}
$17-18$ & $19-20$ & $21-22$ & $23-24$ & $25-26$ & $27-28$ \\
\hline $\mathrm{XX}$ & $\mathrm{XX}$ & $\mathrm{XX}$ & $\mathrm{XX}$ & $\mathrm{XX}$ & $\mathrm{XX}$ \\
\hline NGRP1 & NGRP2 & NGRP3 & NGRP4 & NDT(1) & NDT(2)
\end{tabular}

\begin{tabular}{ccc}
$29-30$ & $31-32$ & $33-34$ \\
\hline $\mathrm{XX}$ & $\mathrm{XX}$ & $\mathrm{XX}$ \\
\hline NDT(3) & NEND1 & NTYPLT
\end{tabular}

$\underline{\text { Symbol }}$

Description of Variables

NBLOCK Block code for curve No. 1.

NBL2 Block code for curve No. 2.

NBL3 Block code for curve No. 3.

NBL4 Block code for curve No. 4.

NDT(I) Index code that specifies what soil moisture data are to be plotted, e.g. Code 1 would be for 0 "- 3 " depth, Code 4,0 "-12" depth when NTYPLT equals 1 (See Table 1).

NEND1 Index code which determines the end of plotting and data summary. The following options are available.

NEND1 $=0$ : Additional curves are to be plotted. After the data specified on parameter card No. 2 are plotted, control is transferred to the next parameter card.

NEND1 = 9: End of plotting. An entirely new group of soil moisture data is to be processed.

NEND1 = 99: End of analysis. All processing terminates.

NGRP2 GROUPS number to be plotted for curve No. 1 where GROUPS is an arithmetic average of the data specified in TRCOMB.

NGRP2

Same as NGRPl except for curve No. 2 . 
TABLE 1 - Codes to Identify the Depth at which Moisture Data is being Plotted

\begin{tabular}{rlll}
\hline $\begin{array}{l}\text { Code } \\
\text { (NDT) }\end{array}$ & $\begin{array}{l}\text { Depths for } \\
\text { Total Accumulated } \\
\text { Soil Moisture } \\
\text { (Inches) }\end{array}$ & $\begin{array}{l}\text { Depths for } \\
\text { Total Soil } \\
\text { Moisture } \\
\text { (Inches) }\end{array}$ & $\begin{array}{l}\text { Total Soil } \\
\text { Moisture for } \\
\text { Groups } \\
\text { (Inches) }\end{array}$ \\
\hline 1 & $0-3$ & $0-6$ & $0-6$ \\
4 & $0-6$ & $6-12$ & $6-12$ \\
3 & $0-9$ & $12-18$ & $12-18$ \\
4 & $0-12$ & $18-24$ & $18-24$ \\
5 & $0-15$ & $24-30$ & $24-30$ \\
6 & $0-18$ & $30-36$ & $30-36$ \\
7 & $0-21$ & $36-42$ & $36-42$ \\
8 & $0-24$ & $42-48$ & $42-48$ \\
9 & $0-27$ & $48-54$ & $48-54$ \\
10 & $0-30$ & $54-60$ & $54-60$ \\
11 & $0-36$ & $60-66$ & $60-66$ \\
12 & $0-42$ & $66-72$ & $66-72$ \\
13 & $0-48$ & & \\
14 & $0-54$ & & \\
16 & $0-60$ & & \\
\hline
\end{tabular}


NGRP3

Same as NGRPl except for curve No. 3 .

NGRP4

Same as NGRPl except for curve No. 4 .

NTYPLT

Index code for plotting option. The options available are:

NTYPLT $=1$ : Plotting of accumulated total soil moisture for specified block-treatment and depth combinations.

NTYPLT $=2$ : Plottings of total soil moisture for specified block-treatment combinations at given 6" increments.

NTYPLT $=3$ : Plottings of combined treatments at given 6" increments.

NTYPLT $=4$ : Plottings of accumulated total soil moisture for the combined treatments.

NTREAT

Treatment code for curve No. 1.

NTRT2

Treatment code for curve No. 2 .

NTRT3

Treatment code for curve No. 3 .

NTRT4

Treatment code for curve No. 4 .

Basic Data

The basic input data normally will be on magnetic tape, and for the system being discussed, result from Section 1 . The input required for Section 1 and the resulting output is given in Appendixes A1, A2, and A3.

The basic input data must be loaded on S.SUOO. The format of this data and a description of the variables follows:

\begin{tabular}{ccccccc}
$7-12$ & $13-15$ & $16-19$ & $21-26$ & $29-31$ & $34-38$ & $39-40$ \\
\hline$+\mathrm{XXXXX}$ & $+\mathrm{XX}$ & $+\mathrm{XXX}$ & $\mathrm{XXXXXX}$ & XXX & XXXX. & XX \\
\hline MO & ND & NYR & ASITE & ATEBE & ATEM1 & ATEM2
\end{tabular}

\begin{tabular}{crc}
$105-116$ & $125-127$ & $128-132$ \\
\hline+ XXXXXXXX.XX & XXX & XXXXX \\
\hline PCMOIS & ANUP & ANCONT
\end{tabular}


ANCONT Sequence number of basic input data.

ANUP Update code.

ASITE Block-treatment identification.

ATEBE Soil moisture access tube identification.

ATEM1 Depth at which soil moisture reading was taken.

ATEM2 Units for the ATEM1 readings. In this program, the units must be either inches or feet. These designations are given as IN for inches and FT for feet.

MO Month

ND Day

NYR Year

PCMOIS Percentage moisture by volume.

\section{PROGRAM CONSTANTS}

A number of program constants are developed within the computer program to facilitate program execution and development. The constants used may be found in the data statements given in Appendix B1. A description of the variable names can be found in Appendix B2.

\section{DATA PREPARATION}

The 4 general types of data that must be prepared prior to the execution of Section 2 are (a) basic input data, (b) identification tables, and (c) two groups of parameter cards. Examples of how these data are prepared for analysis follow.

Basic input data -- The basic input data for Section 2 (Table 2) are obtained from the output of Section 1. The abbreviated sample of data presented in Table 2 is for an experimental study or comparison of the difference in soil moisture content between a conventional tillage practice 
TABLE 2 -- Abbreviated Display of Data Resulting from Section 1 To Be Used
to Ilustrate Data Preparation and Results for Section 2 .

\begin{tabular}{|c|c|c|c|c|c|c|c|c|c|c|c|c|c|c|c|c|}
\hline 1300 & 521 & 66 & NTN1 & 1 & $12 \mathrm{IN}$ & 9477. & 9514. & -0 . & -0 . & -0 . & 18991 . & 9495.5 & 69.17 & 32.00 & IIL & 1 \\
\hline 1300 & 521 & 66 & NTM1 & 1 & $18 \mathrm{IN}$ & -0 . & 10899. & 11052 . & -0 . & -0 . & 21951 . & 10975.2 & 79.406 & 38.37 & IIL & 2 \\
\hline $\begin{array}{l}1300 \\
1300\end{array}$ & $\begin{array}{ll}5 & 21 \\
5 & 21\end{array}$ & $\begin{array}{l}66 \\
66\end{array}$ & NTM1 & 1 & $24 \mathrm{IN}$ & -0. & 11689. & 11756. & -0. & -0 . & 23445 . & 11722.5 & 85.40 & 41.04 & TIL & $\begin{array}{l}3 \\
4\end{array}$ \\
\hline $\begin{array}{l}1300 \\
1300\end{array}$ & $\begin{array}{ll}5 & 21 \\
5 & 21\end{array}$ & $\begin{array}{l}66 \\
66\end{array}$ & $\begin{array}{l}\text { NTN1 } \\
\text { NTM1 }\end{array}$ & 1 & $\begin{array}{l}36 \mathrm{IN} \\
48 \mathrm{IN}\end{array}$ & 10372 . & 10503. & -0 & -0. & -0. & 20875 . & 10437.5 & 10.04 & 36.02 & $\begin{array}{l}\text { IIL } \\
\text { IIL }\end{array}$ & $\begin{array}{l}4 \\
5\end{array}$ \\
\hline 1300 & 521 & 60 & NTN1 & 2 & & 11878. & 11932. & -0. & -0. & -0. & 23810 . & 11905.0 & 86.73 & $\begin{array}{l}42.74 \\
27.31\end{array}$ & IIL & $\begin{array}{l}5 \\
6\end{array}$ \\
\hline 1300 & 521 & 66 & NTMI & 2 & $\begin{array}{l}12 \mathrm{IN} \\
18 \mathrm{IN}\end{array}$ & $\begin{array}{l}8342 . \\
9758\end{array}$ & 8318. & -0 . & -0. & -0. & 16660 . & 8330.0 & $\begin{array}{l}60.68 \\
71.39\end{array}$ & 33.23 & TIL & 7 \\
\hline 1300 & 521 & 66 & NTMI & 2 & & $\begin{array}{l}9758 . \\
-0 .\end{array}$ & $\begin{array}{r}9841 . \\
10953 .\end{array}$ & $\begin{array}{r}-0 . \\
11028\end{array}$ & $\begin{array}{l}-0 . \\
-0\end{array}$ & $\begin{array}{l}-0 . \\
-0\end{array}$ & 21981 : & $\begin{array}{r}9799.5 \\
10990.5\end{array}$ & 80.07 & 36.44 & TIL & 8 \\
\hline 1300 & 521 & 66 & NTM1 & 2 & $36 \mathrm{IN}$ & $12178^{\circ}$. & 12241 . & -0 & -0. & -0. & 24419 . & 12209.5 & 88.95 & 44.16 & IIL & 9 \\
\hline 1300 & 521 & 60 & NTMI & 2 & $48 \mathrm{IN}$ & 11013. & 10964. & -0. & -0 & -0. & 21977. & $10 \rightarrow 88.5$ & 80.65 & 38.43 & IIL & 10 \\
\hline 1300 & 521 & 66 & NTMI & 3 & $12 \mathrm{IN}$ & 8089 . & 8129. & -0 . & -0 . & -0 . & 16218 . & 8109.0 & 59.07 & 26.44 & IIL & 11 \\
\hline 1300 & 521 & 66 & NTN 1 & 3 & $18 \mathrm{IN}$ & 10501. & 10321. & -0 . & -0 . & -0 . & 20822 . & 10411.0 & 75.84 & 35.88 & IIL & 12 \\
\hline 1300 & 521 & 66 & NIMl & 3 & $24 \mathrm{IN}$ & 12126. & 12210. & -0 . & -0 . & -0 . & 24336 . & 12168.0 & 88.64 & 43.92 & IIL & 13 \\
\hline 1300 & 521 & 66 & NIM1 & 3 & $36 \mathrm{IN}$ & 12858 . & 12975. & -0 . & -0 & & 25833 . & 12916.5 & 94.10 & 47.66 & TIL & 14 \\
\hline 1300 & 521 & 66 & NTM1 & 3 & $48 \mathrm{IN}$ & 12875. & 12723. & -0 . & -0 . & -0 . & 25598 . & 12799.0 & 93.24 & 40.99 & IIL & 15 \\
\hline $\begin{array}{l}1300 \\
1300\end{array}$ & $\begin{array}{ll}521 \\
5 & 21\end{array}$ & 66 & CTN1 & 1 & $12 \mathrm{IN}$ & 9796. & & -0 . & -0. & & 19745. & 9872.5 & 71.92 & 33.55 & IIL & $\begin{array}{l}16 \\
17\end{array}$ \\
\hline $\begin{array}{l}1300 \\
1300\end{array}$ & $\begin{array}{ll}5 & 21 \\
5 & 2 k\end{array}$ & 66 & $\begin{array}{l}\text { CTN1 } \\
\text { CTN1 }\end{array}$ & 1 & $18 I N$ & $\begin{array}{l}-0 . \\
12363\end{array}$ & 12007. & 11882 . & -0. & -0 . & 23889 . & 11944.5 & 87.02 & $\begin{array}{l}42 \cdot 71 \\
45.28\end{array}$ & TIL & $\begin{array}{l}17 \\
10\end{array}$ \\
\hline$\frac{1300}{1300}$ & $\frac{5}{5} \frac{2 k}{21}$ & $\frac{66}{60}$ & $\frac{C T N_{1}}{C T N_{1}}$ & $\frac{1}{1}$ & $\begin{array}{l}241 \mathrm{~N} \\
361 \mathrm{~N}\end{array}$ & $12363{ }^{\circ}$ & 12520. & -0. & -0. & -0. & 24883. & 12441.5 & 90.64 & $\frac{45.28}{32.13}$ & IIL & 18 \\
\hline 1300 & 521 & 66 & CTM1 & 1 & $\begin{array}{l}361 \mathrm{~N} \\
481 \mathrm{~N}\end{array}$ & 13822. & 13815. & -0 & -0 . & -0 . & 27037. & $13818^{\circ}$ & 100.67 & $\begin{array}{l}22.13 \\
55.63\end{array}$ & III & 19 \\
\hline 1300 & 521 & 66 & СTMI & 2 & $\begin{array}{l}48 \mathrm{IN} \\
12 \mathrm{IN}\end{array}$ & 14402 . & 14536 . & -0. & -0. & -0 . & 28938 . & 14469.0 & 105.41 & $\begin{array}{l}55.63 \\
29.06\end{array}$ & TIL & 20 \\
\hline 1300 & 521 & 66 & CTMI & 2 & $\begin{array}{l}12 \mathrm{IN} \\
18 \mathrm{IN}\end{array}$ & -0 . & 8770 . & 8783. & -0. & -0 . & 17553. & 8776.5 & 63.94 & $\begin{array}{l}29.06 \\
38.03\end{array}$ & IIL & $\begin{array}{l}21 \\
22\end{array}$ \\
\hline 1300 & 521 & 60 & CIMI & 2 & & 11035. & 11035. & -0. & -0. & -0. & 22070. & 11035.0 & 80.39 & 46.21 & IIL & 23 \\
\hline 1300 & 521 & 66 & CTMI & 2 & $\begin{array}{l}24 \mathrm{IN} \\
36 \mathrm{IN}\end{array}$ & 12590. & 12672 . & $\begin{array}{l}-0 . \\
-0\end{array}$ & $\begin{array}{l}-0 . \\
-0\end{array}$ & $\begin{array}{l}-0 . \\
-0\end{array}$ & $\begin{array}{l}25262 . \\
27767 .\end{array}$ & $\begin{array}{l}12631.0 \\
13883.5\end{array}$ & 101.14 & 52.48 & riL & 24 \\
\hline 1300 & 521 & 66 & CTM1 & 2 & $48 \mathrm{IN}$ & 13672 . & $\begin{array}{l}13040^{\circ} \\
-0\end{array}$ & 13763. & -0 & -0. & 27435 . & 13717.5 & & 51.05 & TIL & 25 \\
\hline 1300 & 521 & 66 & CTMl & 3 & $12 \mathrm{IN}$ & 10286. & 10444. & & -0 . & -0 . & 20730 . & 10365.0 & 75.01 & 35.01 & IIL & 26 \\
\hline 1300 & 521 & 66 & CTM1 & 3 & $18 \mathrm{IN}$ & -0 & 11898. & 11977. & -0 . & -0 . & 23875 . & 11937.5 & 86.90 & 42.88 & IIL & 27 \\
\hline 1300 & 521 & 66 & CTM1 & 3 & $24 \mathrm{IN}$ & 12698 . & 12562 . & -0 . & -0 . & -0 . & 25260 . & 12630.0 & 32.01 & 46.21 & TIL & 28 \\
\hline 1300 & $\begin{array}{ll}521 \\
5\end{array} 2$ & 66 & CTM1 & 3 & $361 \mathrm{~N}$ & 12899. & 12776 . & -0 . & -0 . & -0 . & 25675 . & 12837.5 & 93.52 & 47.22 & IIL & 29 \\
\hline 1300 & 521 & .66 & CTN1 & 3 & $48 \mathrm{IN}$ & 12236. & 12427. & -0 . & -0 . & -0 . & 24663 . & 12331.5 & 89.84 & 44.70 & IIL & 30 \\
\hline 1300 & 521 & 66 & NTM2 & 3 & $12 \mathrm{IN}$ & 8981 . & 9128. & -0 . & -0. & -0 . & 18109. & 9054.2 & 63.90 & $30 \cdot 28$ & TIL & 31 \\
\hline $\begin{array}{l}1300 \\
1300\end{array}$ & 521 & $\begin{array}{l}66 \\
66\end{array}$ & NTM2 & 3 & $18 \mathrm{IN}$ & 11193. & 11277 . & -0 . & -0. & -0 . & 22470 . & 11235.0 & 31.85 & 39.61 & IIL & $\begin{array}{l}32 \\
33\end{array}$ \\
\hline & 521 & 66 & NTM2 & 3 & $24 \mathrm{IN}$ & 12721. & 12522. & -0 . & -0 . & -0. & 25243. & 12621.5 & 91.45 & 40.16 & IL & 33 \\
\hline 1300 & $\begin{array}{ll}5 & 21 \\
5 & 21\end{array}$ & 66 & $\begin{array}{l}\text { NTM } 4 \\
\text { NTM }\end{array}$ & 1 & $12 \mathrm{IN}$ & $6544^{\circ}$ & -0 . & -0 & -0. & -0 & 6544. & 6544.0 & 47.07 & 20.80 & IIL & 116 \\
\hline $\begin{array}{l}1300 \\
1300\end{array}$ & $\begin{array}{ll}5 & 21 \\
5 & 21\end{array}$ & $\begin{array}{l}00 \\
60\end{array}$ & $\begin{array}{l}\text { NTM4 } \\
\text { NTM4 }\end{array}$ & $\begin{array}{l}1 \\
1\end{array}$ & $\begin{array}{l}18 \mathrm{IN} \\
24 \mathrm{IN}\end{array}$ & $\begin{array}{r}7835 . \\
11330 .\end{array}$ & $\begin{array}{l}-0 . \\
-0\end{array}$ & $\begin{array}{l}-0 . \\
-0\end{array}$ & $\begin{array}{l}-0 . \\
-0\end{array}$ & $\begin{array}{l}-0 . \\
-0\end{array}$ & $\begin{array}{r}7835 . \\
11330 .\end{array}$ & $\begin{array}{r}7835.0 \\
11330.0\end{array}$ & $\begin{array}{l}57.08 \\
82.34\end{array}$ & $\begin{array}{l}25.43 \\
40.02\end{array}$ & $\begin{array}{l}\text { TIL } \\
\text { TIL }\end{array}$ & $\begin{array}{l}117 \\
118\end{array}$ \\
\hline 1300 & 521 & 66 & NTN4 & 1 & $36 \mathrm{IV}$ & 13338. & -0. & -0 . & -0. & -0 . & 13338 . & 13338.0 & 97.17 & 49.73 & IIL & 119 \\
\hline 1300 & 521 & 66 & NTN4 & 1 & $48 \mathrm{IN}$ & 13950. & -0 . & -0 . & -0 & -0 & 13950 . & 13950.0 & 101.63 & 52.83 & IIL & 120 \\
\hline 1300 & 530 & 66 & NTNI & 3 & $12 \mathrm{IN}$ & 8291. & 8306 . & -0 & -0 . & -0 . & 10657. & 8328.5 & 59.04 & 20.78 & IIL & 121 \\
\hline 1300 & 530 & 66 & NTMI & 3 & $18 \mathrm{IN}$ & 10968. & 10912 . & -0 . & -0 & -0 & 21880 . & 10940.0 & 78.34 & 37.40 & TIL & 122 \\
\hline 1300 & 530 & 66 & NTMI & 3 & $24 \mathrm{IN}$ & 12145 . & 12260. & -0 . & -0 . & -0 . & 24405 . & 12202.5 & 87.38 & 43.13 & IIL & 123 \\
\hline 1300 & 530 & 66 & NTN1 & 3 & $36 \mathrm{IN}$ & 13117. & & 13071 . & -0 . & -0 . & 26188 . & 13094.0 & 93.16 & 47.41 & ril & 124 \\
\hline 1300 & 530 & 66 & NTN1 & 3 & $48 \mathrm{IN}$ & $12834^{\circ}$ & 12793. & -0 & -0 . & -0. & 25627 . & 12813.5 & 91.75 & 46.00 & IIL & 125 \\
\hline 1300 & 530 & 66 & $\mathrm{CTN}_{4}$ & 3 & $301 \mathrm{~N}$ & 15005. & -0 . & -0 & -0 & -0 & 15005. & 13005.0 & 107.44 & 57.16 & TIL & 239 \\
\hline 1300 & & 66 & CIN4 & 3 & $48 \mathrm{IN}$ & $14625^{\circ}$. & -0. & -0. & $-c$. & -0 & 14625 . & 14625.0 & & 55.13 & IIL & 240 \\
\hline 1300 & 530 & 66 & CIM4 & & o Iiv & -0 & -0 . & 5934. & 5931. & -0 . & 11865. & 5932.0 & 58.95 & 10.36 & IIL. & 241 \\
\hline 1300 & 530 & 66 & NIN4 & & $O \mathrm{IN}$ & -0 & -0 . & 7376. & 7186. & -0 . & 14562 . & 1281.0 & 72.35 & 21.58 & IIL & 242 \\
\hline 1300 & 530 & 66 & $N_{1} N_{3}$ & & OIN & -0 . & -0 . & 7309 . & 7330. & -0 . & 14039. & 7319.5 & 72.74 & 21.09 & IIL & 243 \\
\hline 1300 & 530 & 66 & $\mathrm{CTN}_{3}$ & & $01 \mathrm{v}$ & -0 . & -0 . & 6228. & 6052. & -0 . & 12280 . & 0140.0 & 61.02 & 12.01 & TIL & 244 \\
\hline 1300 & 530 & 66 & CTN2 & & OIN & -0 & -0. & 6321. & 6155. & -0 . & 12476. & 6238.0 & 01.49 & 12.77 & TIL & 245 \\
\hline 1300 & 530 & 66 & NTN2 & & OIN & -0 . & -0 . & 7437. & 7263. & -0 . & 14700 . & 7350.0 & 73.04 & 22.13 & IIL & 246 \\
\hline 1300 & 530 & 60 & CTN1 & & $O I N$ & -0 . & -0 . & 6078 . & 6009 . & -0 . & 12141. & 6073.5 & 60.35 & 11.48 & IIL & 241 \\
\hline 1300 & 530 & 66 & NTNI & & OIN & -0. & -0. & 7217. & 7279. & -0. & 14496. & 7248.0 & 72.03 & 21.32 & IIL & 248 \\
\hline 1300 & 611 & 66 & NTN 1 & 3 & $12 \mathrm{IN}$ & 7913. & 7953. & -0. & -0. & -0 . & 15800. & 7933.0 & 56.19 & 25.28 & IIL & 249 \\
\hline 1300 & 611 & 66 & INTN1 & 3 & $18 \mathrm{IN}$ & 10432 . & 10471 . & -0 & -0. & -0 . & 20903. & 10451.3 & $74.8 ?$ & 35.27 & IIt & $22 n$ \\
\hline 1300 & 611 & 66 & TIN1 & 3 & $\begin{array}{l}24 \mathrm{IN} \\
36 \mathrm{IN}\end{array}$ & $\begin{array}{l}-0 . \\
-0\end{array}$ & $\begin{array}{l}12177 . \\
12892 \text {. }\end{array}$ & $\begin{array}{l}12168 . \\
13004 .\end{array}$ & $\begin{array}{l}-0 . \\
-0 .\end{array}$ & $\begin{array}{l}-0 . \\
-0\end{array}$ & $\begin{array}{l}243450 \\
25896 .\end{array}$ & $\begin{array}{l}12172.5 \\
12948.0\end{array}$ & $\begin{array}{l}87.14 \\
92.09\end{array}$ & $\begin{array}{l}42.99 \\
46.02\end{array}$ & TIL & $\begin{array}{l}251 \\
25,52\end{array}$ \\
\hline $\begin{array}{l}1300 \\
1300\end{array}$ & $\begin{array}{lll}6 & 11 \\
6 & 11\end{array}$ & $\begin{array}{l}60 \\
60\end{array}$ & $\begin{array}{l}\text { NTN1 } \\
\text { NTN1 }\end{array}$ & 3 & $\begin{array}{l}36 \mathrm{IN} \\
48 \mathrm{IN}\end{array}$ & 12865. & $\begin{array}{l}12892 . \\
13007 .\end{array}$ & 13004. & $-c$. & -0. & 25872 . & 12936.0 & 92.01 & 46.57 & IIL & 203 \\
\hline
\end{tabular}




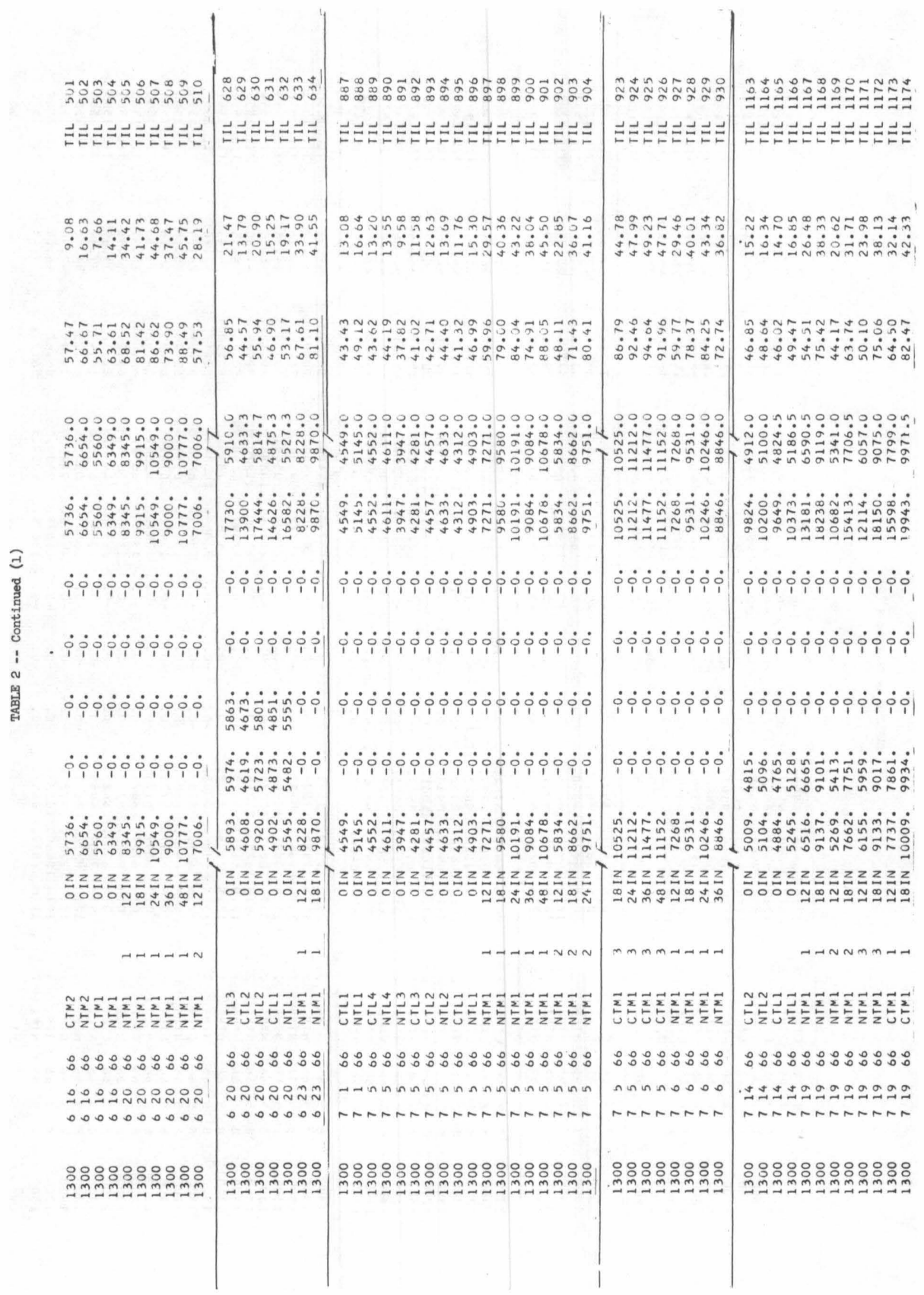




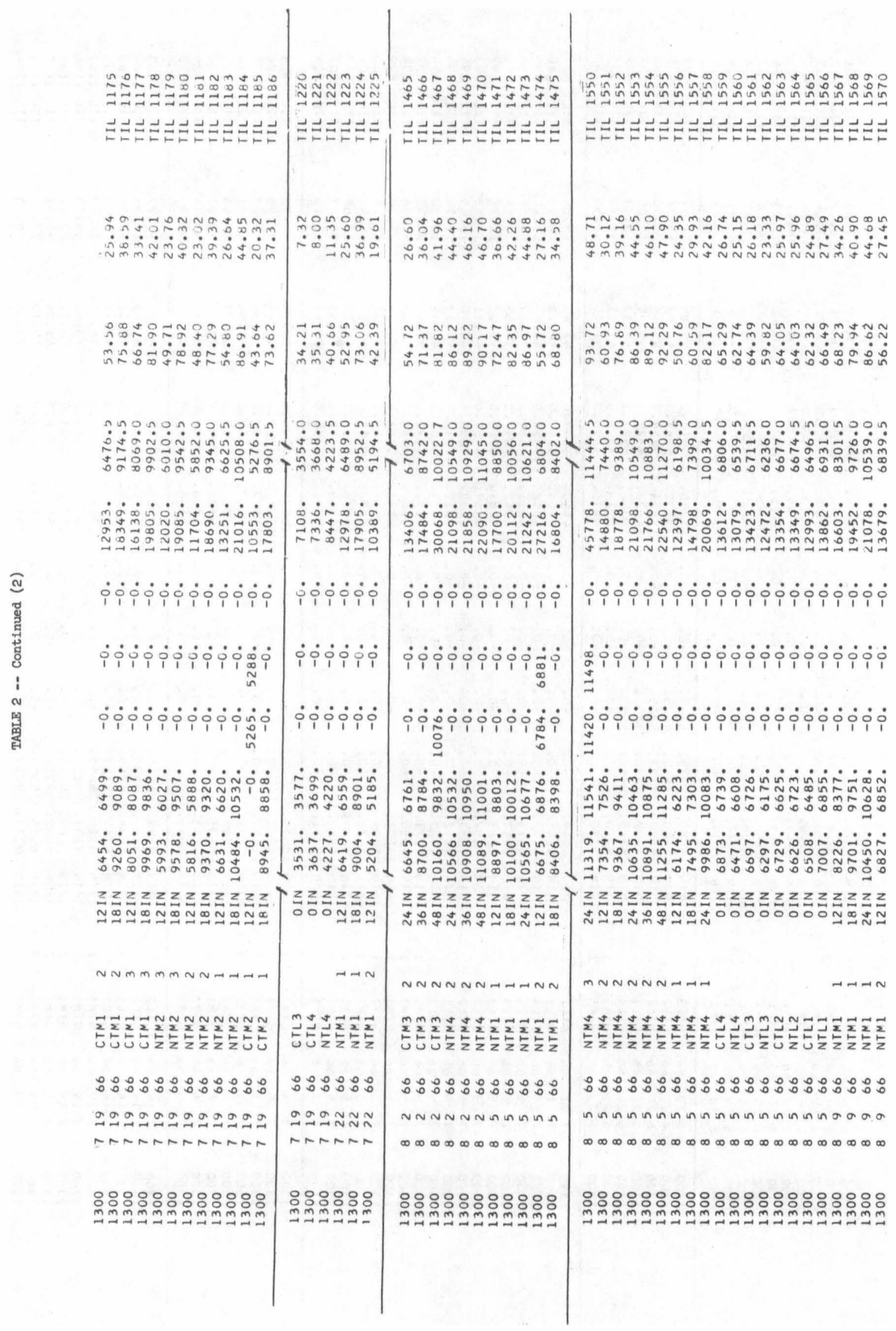


TABLE 3 -- Identifications and Corresponding Coding for Various Block-Treatment Combinations, Depths, and Access Tubes for No-Tillage Experiment Located at Blacksburg, Virginia.

\begin{tabular}{|c|c|c|c|}
\hline Title & Identification & & de \\
\hline Block-Treatment & $\begin{array}{c}\text { (TPFORM) } \\
\text { NTM1 } \\
\text { CTM1 } \\
\text { NTM2 } \\
\text { CTM2 } \\
\text { NTM3 } \\
\text { CTM3 } \\
\text { NTM4 } \\
\text { CTM4 } \\
\text { NTL1 } \\
\text { CTL1 } \\
\text { NTL2 } \\
\text { CTL2 } \\
\text { NTL3 } \\
\text { CTL3 } \\
\text { NTL4 } \\
\text { CTL4 }\end{array}$ & $\begin{array}{c}\text { (TRCODE) } \\
1 \\
2 \\
1 \\
2 \\
1 \\
2 \\
1 \\
2 \\
1 \\
2 \\
1 \\
2 \\
1 \\
2 \\
1 \\
2\end{array}$ & $\begin{array}{c}\text { (BKCODE) } \\
1 \\
1 \\
2 \\
2 \\
3 \\
3 \\
4 \\
4 \\
1 \\
1 \\
2 \\
2 \\
3 \\
3 \\
4 \\
4\end{array}$ \\
\hline Depth & $\begin{array}{c}\text { (DEPTHT) } \\
0 \\
12 \\
18 \\
24 \\
36 \\
48\end{array}$ & $\begin{array}{c}\text { (DEPCOD) } \\
1 \\
2 \\
3 \\
4 \\
5 \\
6\end{array}$ & $\begin{array}{c}\text { (CONV) } \\
6 \\
9 \\
6 \\
9 \\
12 \\
12\end{array}$ \\
\hline Access Tube & $\begin{array}{c}\text { (TUBET) } \\
1 \\
1 \\
1 \\
1 \\
2 \\
2 \\
2 \\
3 \\
3\end{array}$ & $\begin{array}{c}\text { (TUBCOD) } \\
1 \\
1 \\
1 \\
2 \\
2 \\
2 \\
3 \\
3 \\
3\end{array}$ & \\
\hline
\end{tabular}


and a no-tillage practice. The experiment had 2 treatments that were blocked (replicated) 4 times.

Identification tables -- The procedures for the development of the 3 identification tables are discussed on page 4 , however, an example will be given for clarification. The identifications that were arbitrarily assigned to the block-treatment combination at the initiation of the experiment are given in Table 3. Initial assignments were made on a schematic layout of the experimental design (Figure 1).

Soil moisture data were obtained at the surface $(0 "), 12 ", 18 ", 24 "$, $36 "$, and 48 " depths. Only one surface reading was obtained per treatment, and this reading was assumed to be representative for the entire treatment area. These readings were obtained on NLT and CTL plots and were coded identically as corresponding NTM and CTM plots. The depths or levels of moisture sampling were identified as $0,12,18,24,36$, and 48 respectively. These 6 levels were coded sequentially as shown in Table 3 (DEPCOD). CONV values were obtained by linear interpolation between successive sampling points.

Three soil moisture access tubes were located in each plot and were identified as 1, 2, and 3. Any alphabetic, numeric or alphameric identification can be used (e.g. A, B, and C). The only limitation is that the number of characters making up each tube identification cannot exceed 3 . The TUBET values are presented in Table 3 in 3 different locations where each location has the same TUBCOD code. This arrangement is shown to illustrate the coding parameters when TUBET has not been right justified. Routines developed in Section 1 automatically right justify TPFORM,DEPTH and TUBET, which normally eliminates the need for such an arrangement.

The integer code values assigned to the 3 tables are given in Table 3 as TRCODE, BKCODE, DEPCOD, and TUBCOD. The data presented in Table 3 have been placed on data sheets for key punching (Table 4).

Before coding the parameter cards, the variables in each must be studied as to their functions and the desired output decided upon. Examples of several options or alternatives are given in Table 5 to illustrate the utility of the program.

Example 1:

In this example, it is assumed that a complete analysis is desired, i.e. the data must be coded and punched for analysis of variance and all possible total soil moisture summaries and plottings made. 

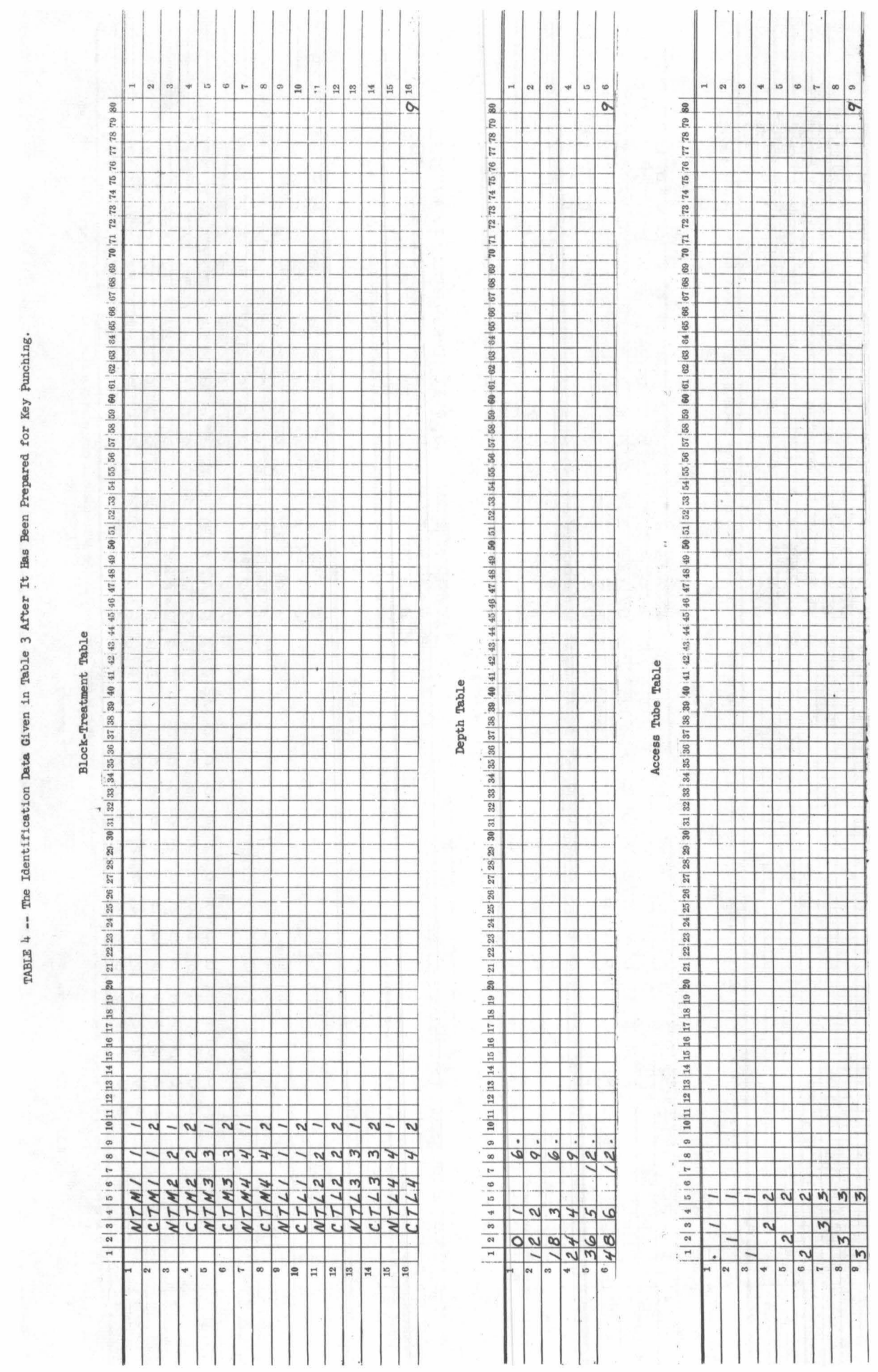

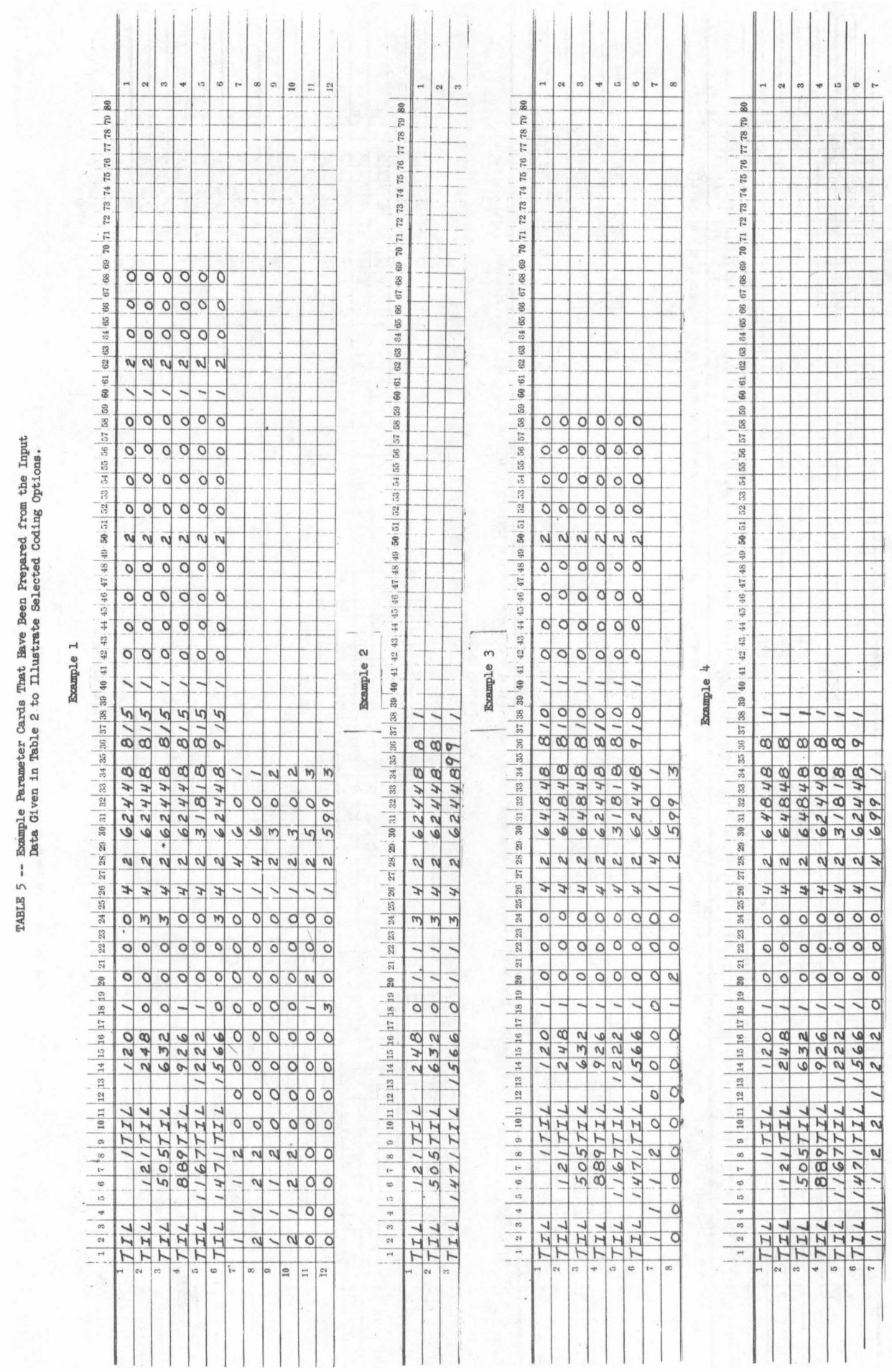
The first and second group of parameter cards would be coded as illustrated in Table 5. Note that the data example has been selected to illustrate the procedure involved when both full and partial records are involved. For the analysis of variance procedure illustrated, no missing records are permitted; therefore, the 24" depth is the maximum depth to be included for this analysis. All data can be plotted regardless of the restrictions placed on the analysis of variance routine. Reference to the discussion starting on page 3 will clarify questions concerning coding.

Example 2:

Example 2 illustrates the coding required when only analysis of variance is desired. Note that the second group of parameter cards is not required.

Example 3:

Example 3 illustrates the coding required with no analysis of variance, but summaries and plotting are desired for total accumulated soil moisture and group combinations. Two curves are compared.

\section{Example 4:}

Example 4 illustrates the parameter cards required for 4 curves per plotting for example 3 ; but no treatment combination.

\section{ORDER OF INPUT}

The order of input for the IBM 7040/1401 Computer is as follows:

a. $\$ \mathrm{JOB}$ card (computer residence card)

b. \$IBJOB card (computer residence card)

c. Binary deck (program instructions)

d. \$ENTRY card (computer residence card)

e. Header card

f. Soil moisture tension table (required when available moisture determined)

g. Block-treatment table

h. Depth table

i. Access tube table

j. First group of parameter cards 
k. Second group of parameter cards

1. Repeat steps e-i where applicable

Note that unless an entirely new experimental study is to be processed steps e through $\mathrm{k}$ will not be repeated. Also, note that the basic input data to Section 2 are on magnetic tape, which must be mounted on S.SUOO.

\section{OUTPUT}

The output from the system can be in many forms depending on the option specified. The form of the output from Section 1 is illustrated in Appendixes A1, A2, and A3. An example of the output that can be obtained from Section 2 is given in Tables 6, 7, 8, 9, 10, 11, 12, 13, 14, 15, and 16 . The example is also the output that would result from the coding given in Table 5 .

The data given in Table 6 are simply a display of the identification tables that were sorted in the computer for the run. This output is used to double-check the identification table for errors or omissions.

If the identification for a given input value cannot be located in the dictionary, then the missing value is written on an output tape in the form given in Table 7 . This routine aids in locating errors that have occured during key punching.

Table 8 is a listing of the punch cards that have been prepared for an analysis of variance. This listing is in the exact order that the data were encountered on the input tape (S.SUOO). Table 9 is a listing of the same data after rearrangement by a sorter for an analysis of variance for a complete cross classified statistical design.

The format specification for the punch card output followed by a description of the variables is given below.

\begin{tabular}{cccccc}
$1-2$ & $3-4$ & $5-6$ & $7-8$ & $9-10$ & $20-25$ \\
\hline $\mathrm{XX}$ & $\mathrm{XX}$ & $\mathrm{XX}$ & $+\mathrm{X}$ & $+\mathrm{X}$ & $+\mathrm{XXXX}$ \\
\hline OUTIDX(1) & OUTIDX(2) & OUTIDX(3) & N5 & OUTIDX(4) & NW
\end{tabular}

\begin{tabular}{ccccc}
$50-52$ & $53-55$ & $56-58$ & $73-75$ & $76-80$ \\
\hline$+\mathrm{XX}$ & $+\mathrm{XX}$ & $+\mathrm{XX}$ & $\mathrm{XXX}$ & $+\mathrm{XXXX}$ \\
\hline MO & ND & NYR & ANUP & NSEQ
\end{tabular}


mo mon mon mom mom mo mo mo

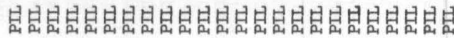

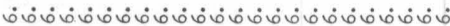

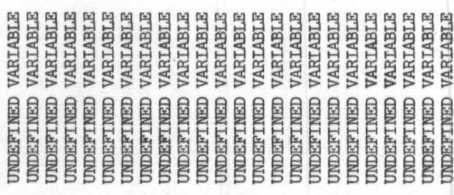




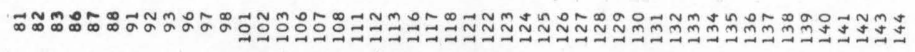

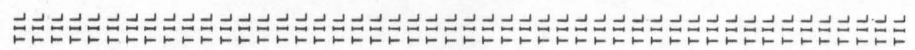

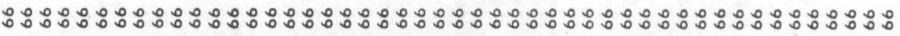

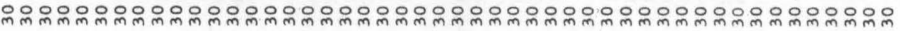

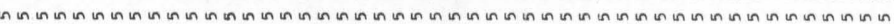

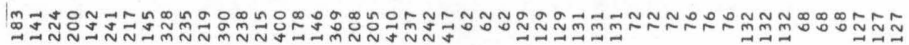

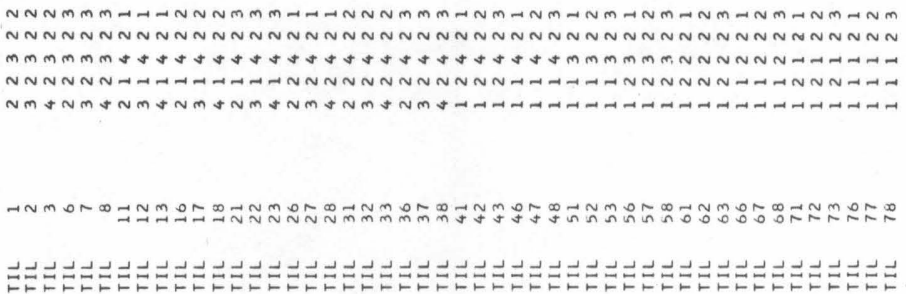

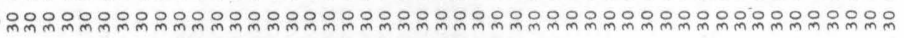

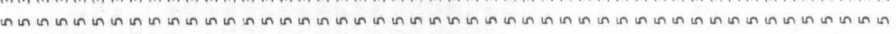

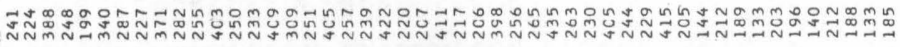




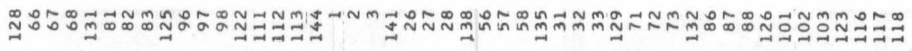

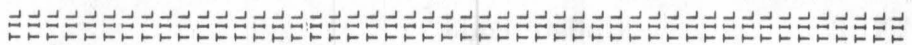

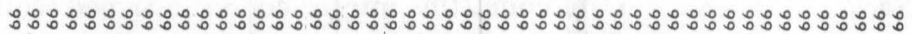

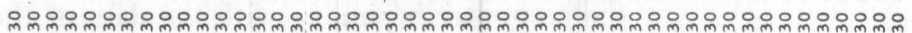

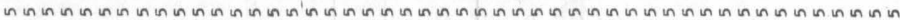

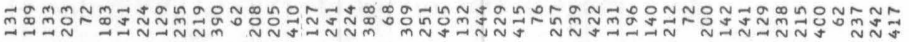

NNNNNNNNNNNNNNNNMMMMMMMMMMMMMMMMMMMMMMMMMMMMMMMM NNNNNNNNNNNNNNNNNNNNNNNNNNNNNNNNNNNNNNNNNNNNNNNN

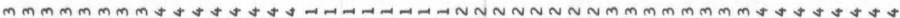

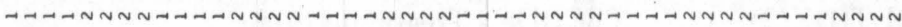

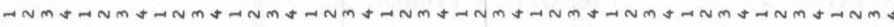

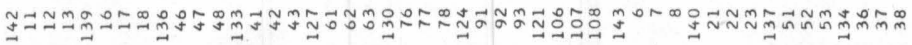

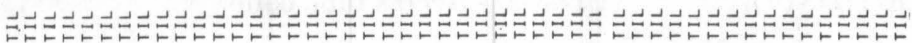

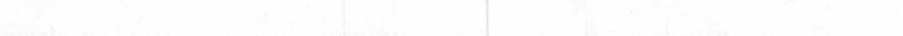

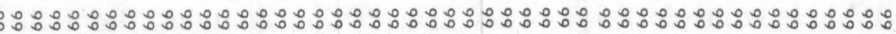

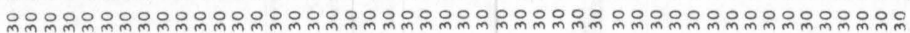
in in in in in in in in in in in in un in in in in in in in in in in in in in in in in un in in in in in in in in in in in in in in in in in in

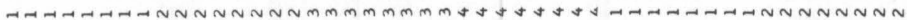

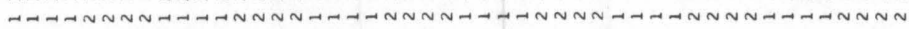

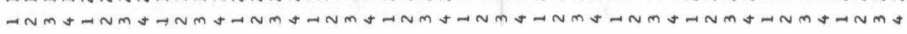


ANUP Update code

MO Month

N5 Date code (this integer code is assigned sequentially by the computer beginning with the date of the first group of observations as 1).

ND Day

NSEQ Sequence number

NW Observed moisture content times 100 for the applicable range in the soil profile (See CONV Table 4)

NYR Year

OUTIDX(1) DEPCOD - Depth Code

OUTIDX(2) TRCODE - Treatment Code

OUTIDX(3) BKCODE - Block Code

OUTIDX(4) TUBCOD - Access tube code

If the punch card data is not needed (no statistical analysis to follow), a listing of the data can be obtained (Table 10) which will show the coding and the conversion of percentage moisture to inches of water. Variable KUNIT1 controls this option. The listing contains the following output.

Column Heading

$\begin{array}{cll}\text { From Table 10 } & \frac{\text { Symbol }}{\text { OUTIDX(1) }} & \text { Description } \\ 1 & \text { DEPCOD } \\ 2 & \text { OUTIDX(2) } & \text { TRCODE } \\ 3 & \text { OUTIDX(3) } & \text { BKCODE } \\ 4 & \text { N5 } & \text { Date Code } \\ 5 & \text { OUTIDX(4) } & \text { TUBCOD } \\ 6 & \text { WATER } & \text { Observed moisture content } \\ & & \text { in inches for applicable } \\ & & \text { soil region }\end{array}$




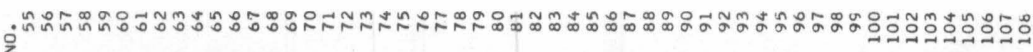
㟧

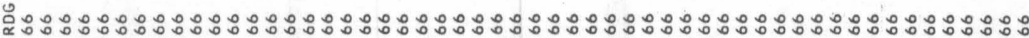

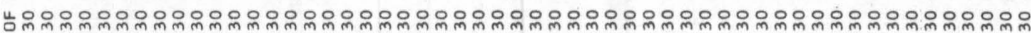

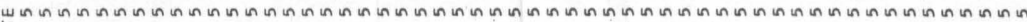
造

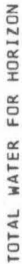

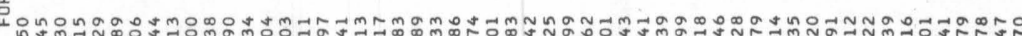

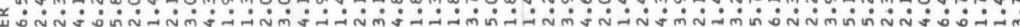

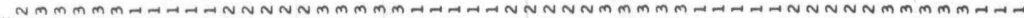
L

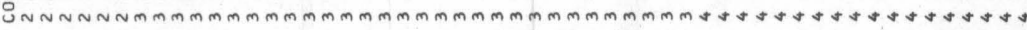

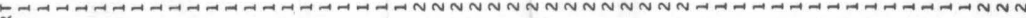

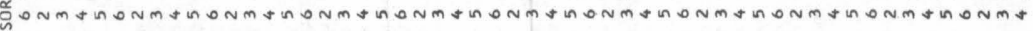

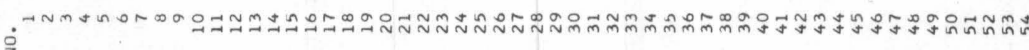

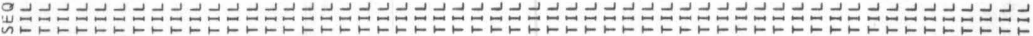

完:

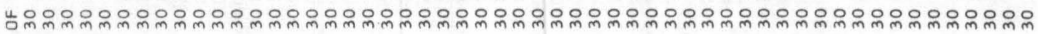

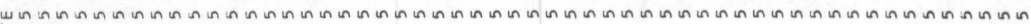
$\stackrel{\longleftarrow}{\square}$

곰

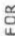

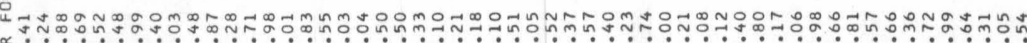

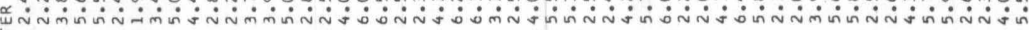
急

崖

mmmmm

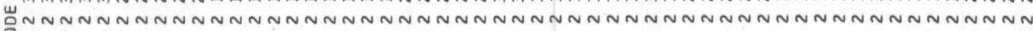

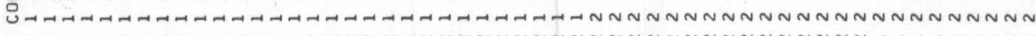

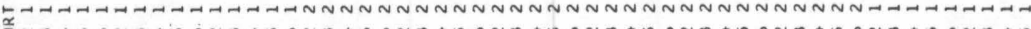

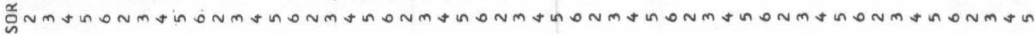




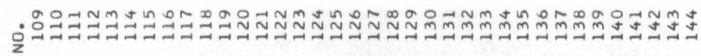

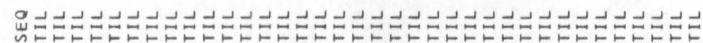

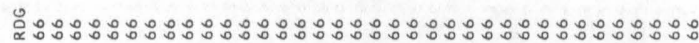

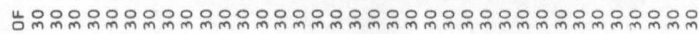

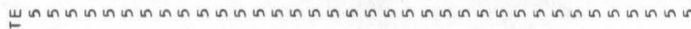
เั

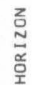

范

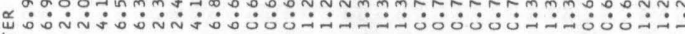
离

峁

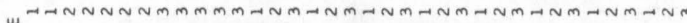

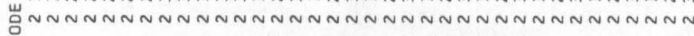

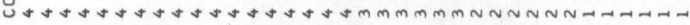

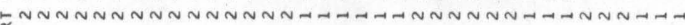

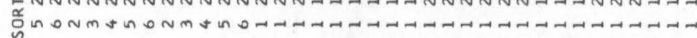




\begin{tabular}{rl|l}
7 & MO & Month \\
8 & ND & Day \\
9 & NYR & Year \\
10 & ANUP & Update Code \\
11 & ANCONT & Sequence Number
\end{tabular}

An example of total accumulated soil moisture summary for specified increments is given in Table 11. The information contained in each column is identified by appropriate column headings. The variable name associated with each column of output, identified from left to right is given below:

Symbol

Description of Variables

I

Block Code

$\mathrm{J}$

Treatment Code

$\operatorname{ACSM}(\mathrm{K}) \quad$ Total accumulated soil moisture present in the soil $\mathrm{K}=1,17$ profile for a given block and treatment combination.

MO Month

ND Day

NYR Year

The total soil moisture for 6" increments is given in Table 12. The variable name associated with each column of output, identified from left to right, is given below:

Symbol

Description of Variables

I

J

ACC(LOM)

$\mathrm{LOM}=1,12$

MO

Month

ND

Day

NYR
Block Code

Treatment Code

Total soil moisture for specified 6" increments. 


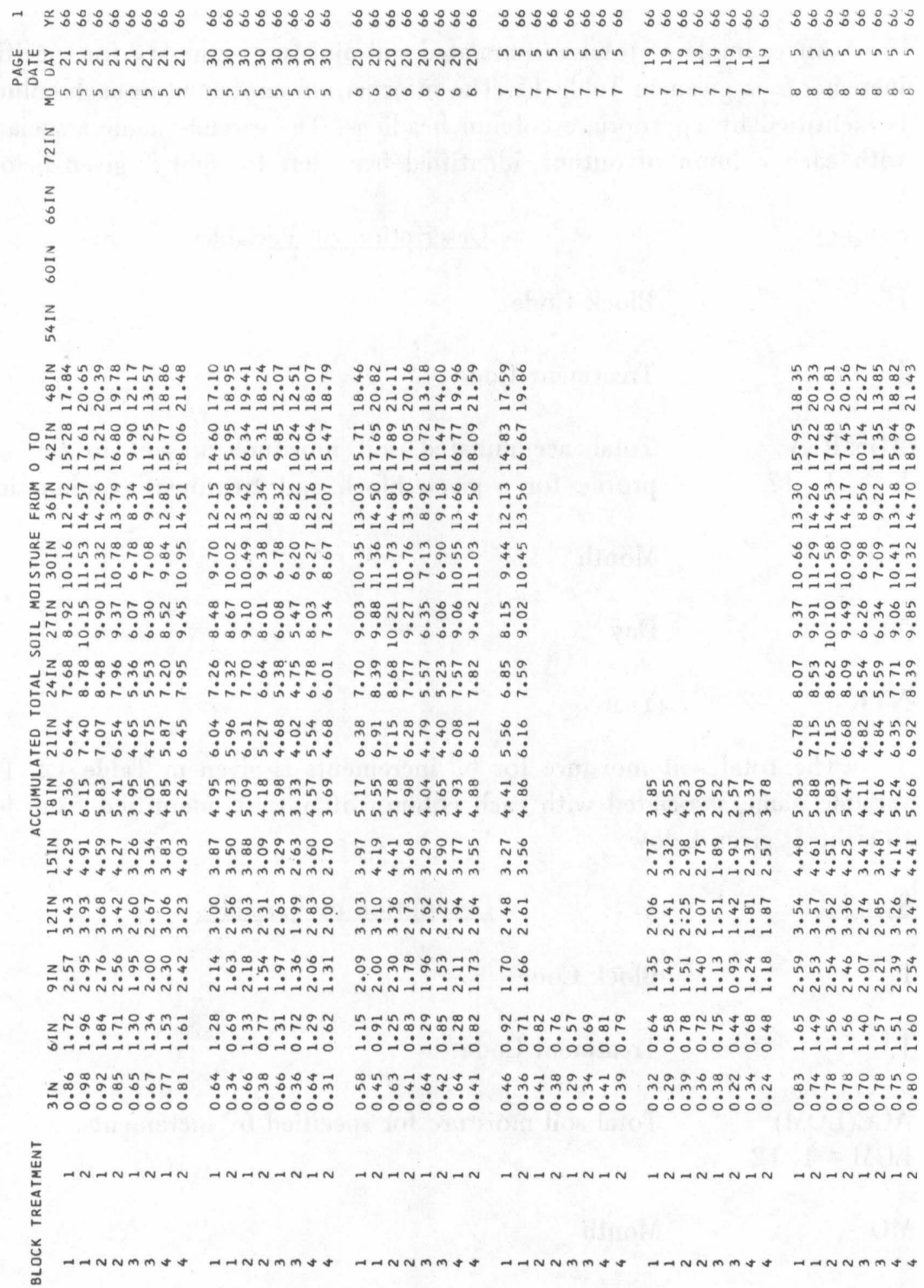


ํํํํํํํํำ

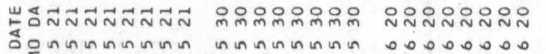

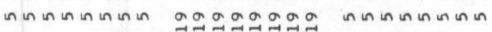

वृ

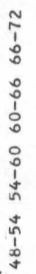

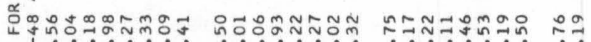

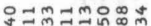

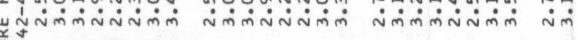

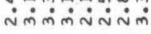

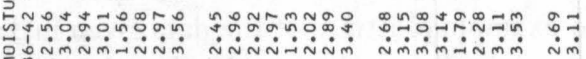

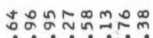

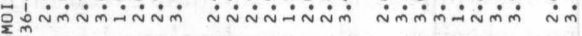

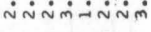

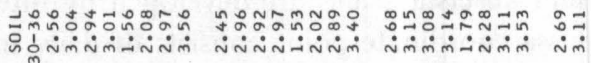

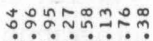

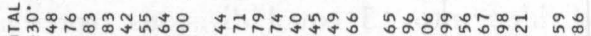

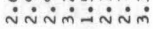

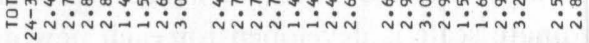

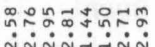

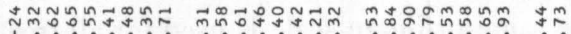

inising

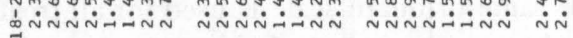

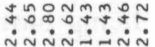

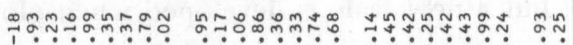

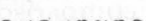

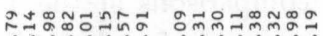

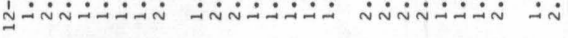

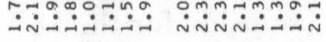

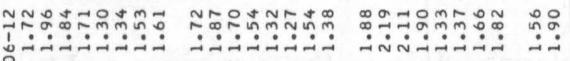

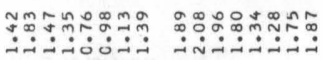

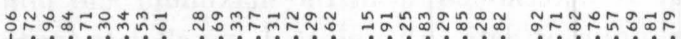

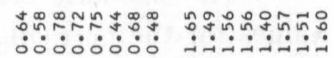

畟

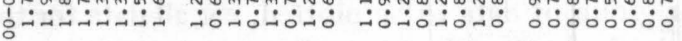

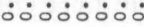

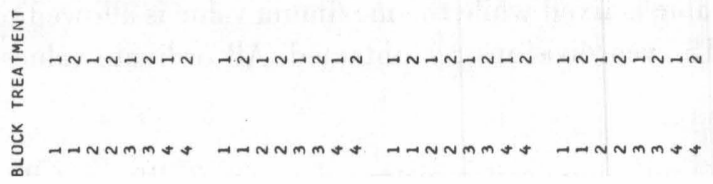

$\rightarrow n \rightarrow n \rightarrow n \rightarrow n \rightarrow n \rightarrow n \rightarrow n \rightarrow n$

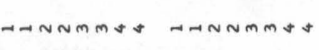


The total soil moisture for the average of various block-treatment combinations is given in Table 13. The variable name associated with each column of output identified from left to right follows:

Symbol

\section{Description of Variables}

NGP

Group Identification

NT(I)

$\mathrm{I}=1,5$

Treatments that have been summed over the entire experiment and averaged to form NGP.

GRPS(I)

$\mathrm{I}=1,12$

Average total soil moisture for specified depths.

MO

Month

ND

Day

NYR

Year

Tables 14, 15, and 16 are example plottings of the data given in Tables 11,12 , and 13. The ordinate and abscissa scales are developed by internal programming routines. The abscissa coordinate point consists of daily increments. The ordinate scale is divided into 15 equal divisions representing inches of water. A different ordinate scale is developed for each new depth being plotted. For example, the same ordinate scale is used for all treatment comparisons at the 12 " depth, but a new scale is developed when plottings are made for the 18 " depth.

The ordinate scale is determined from the maximum and minimum values for the particular group of data to be plotted, e.g.all data for the 12" depth. The minimum value is fixed while the maximum value is allowed to be shifted upwards until 15 even divisions are obtained. All ordinate values are to the nearest .01 ".

Four months of continuous soil moisture data for 3 different depths can be plotted on standard 14-7/8" x 11 "paper. When the data exceeds these limits, the plotting is simply continued on the next page. Plotting is achieved by identifying coordinate points through which a curve may be quickly hand traced. In this program, a maximum of 4 curves may be plotted on 1 graph for comparison (Table 17). The coordinate point identification for each curve is given in Table 18: 


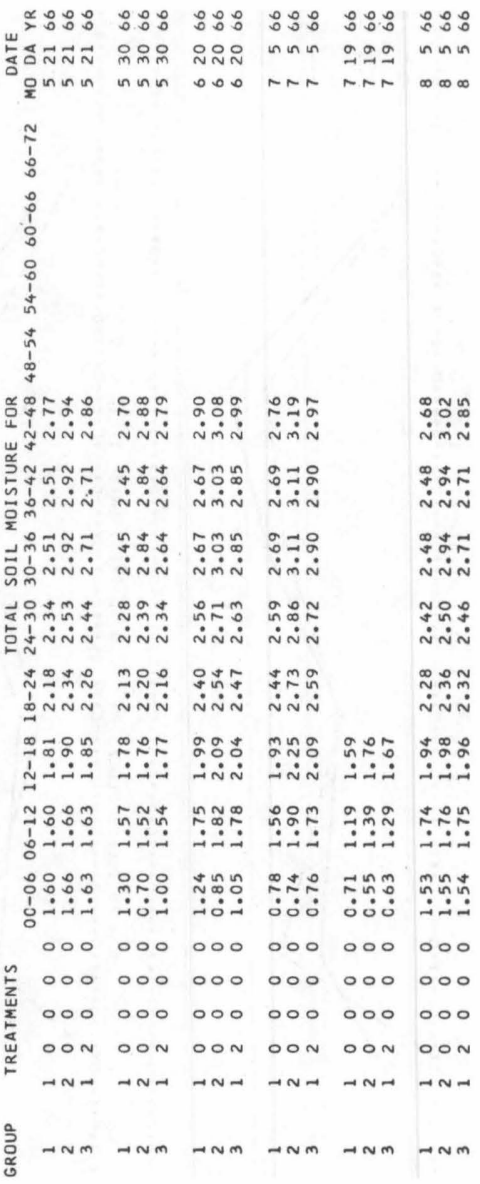




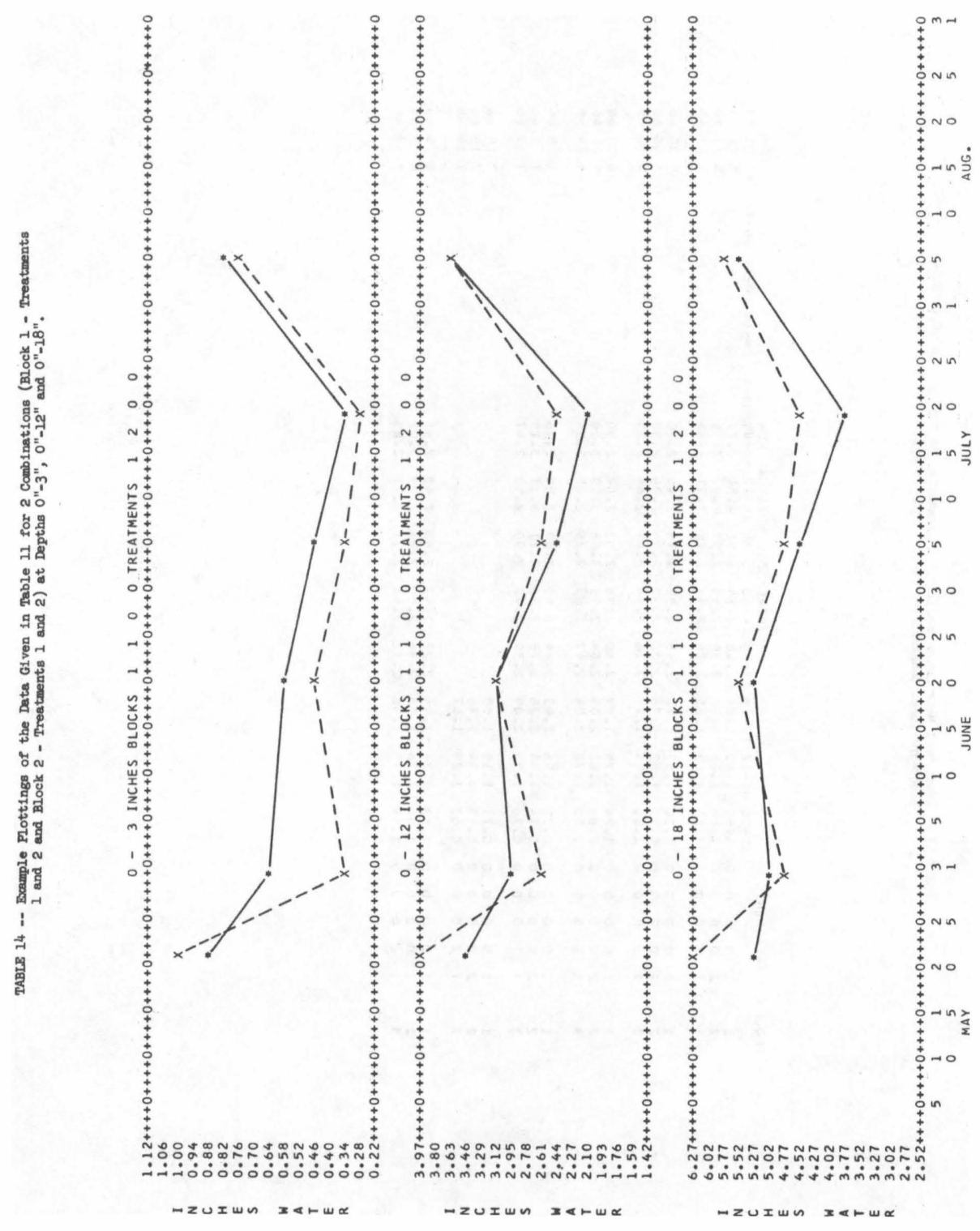




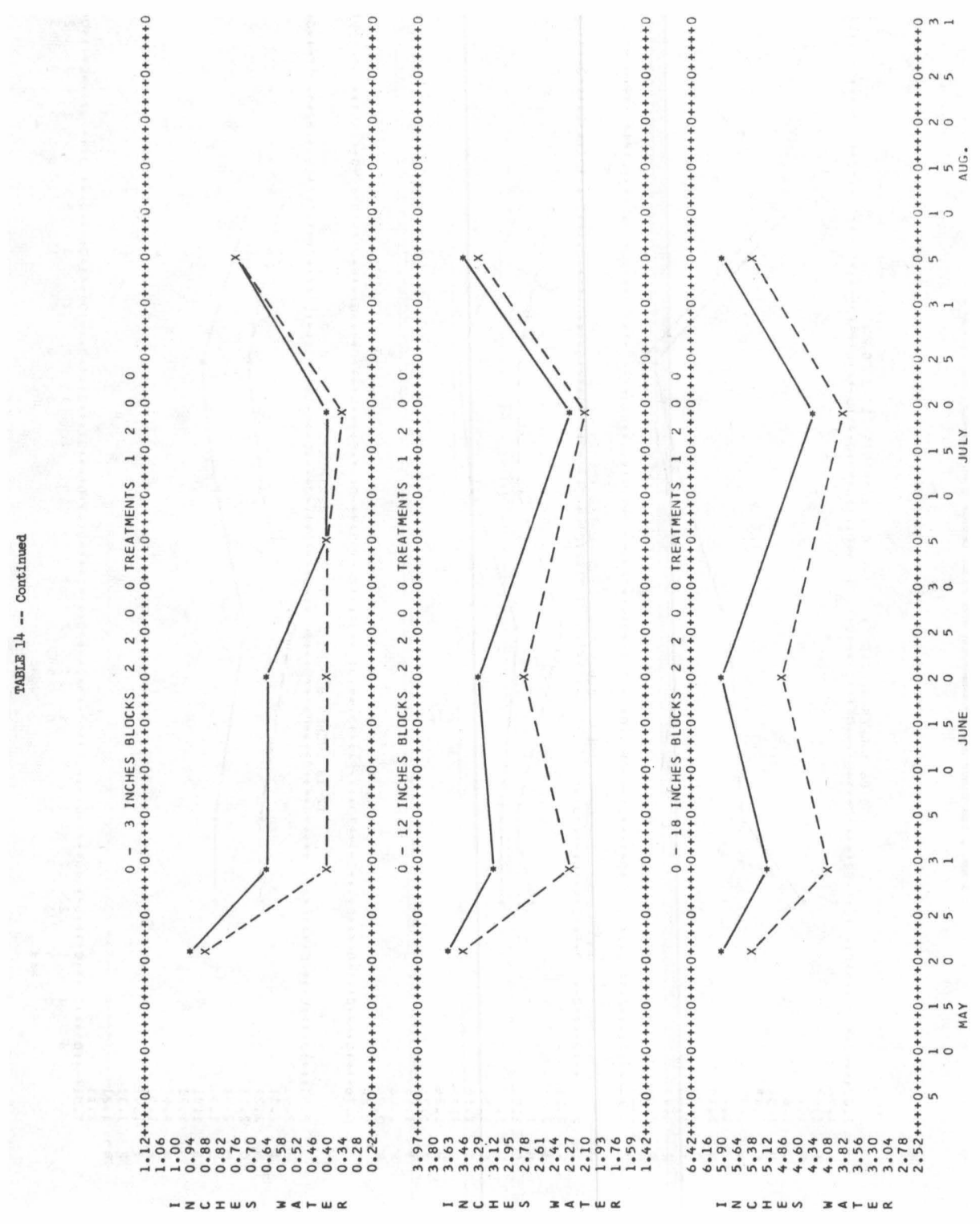




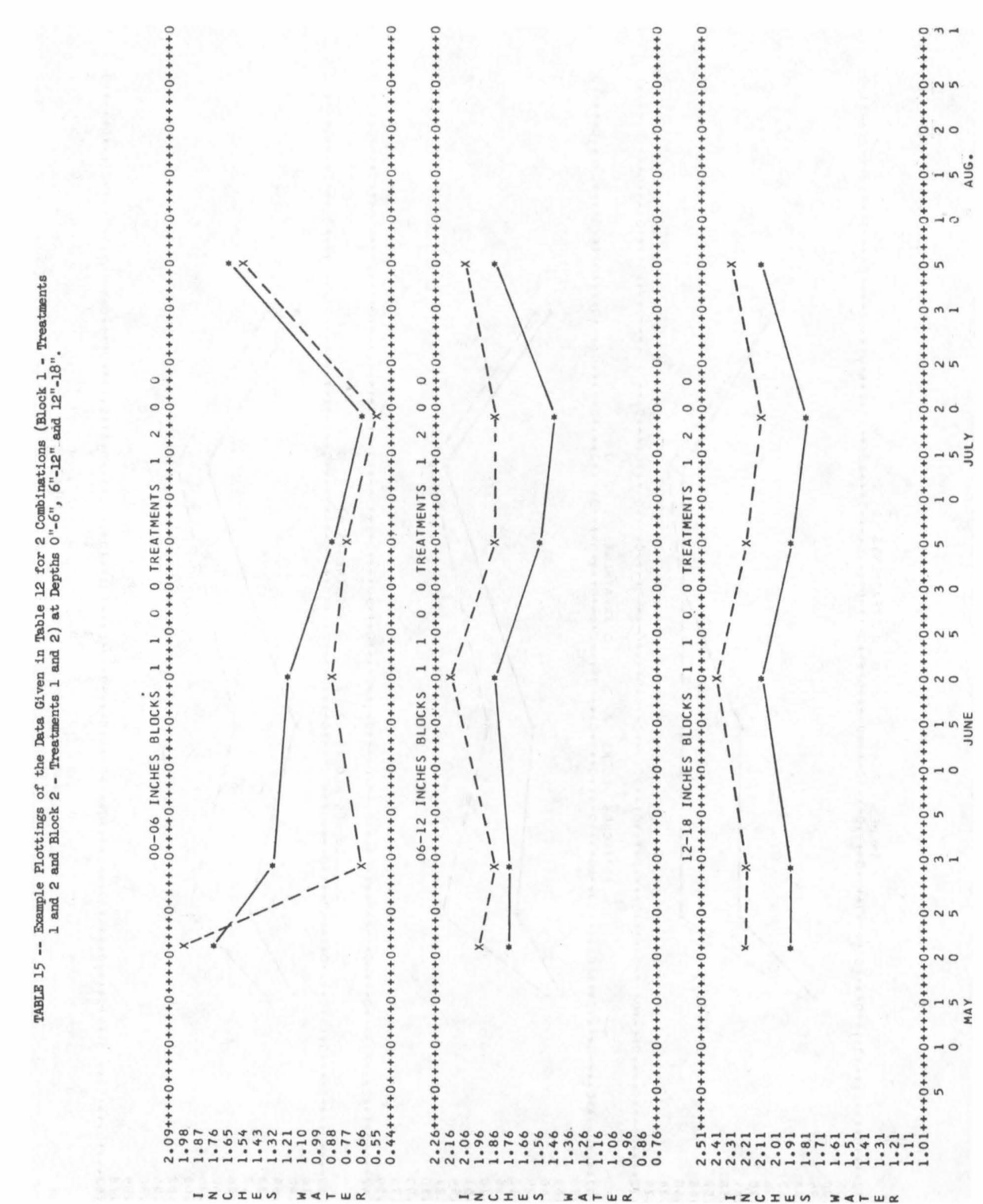



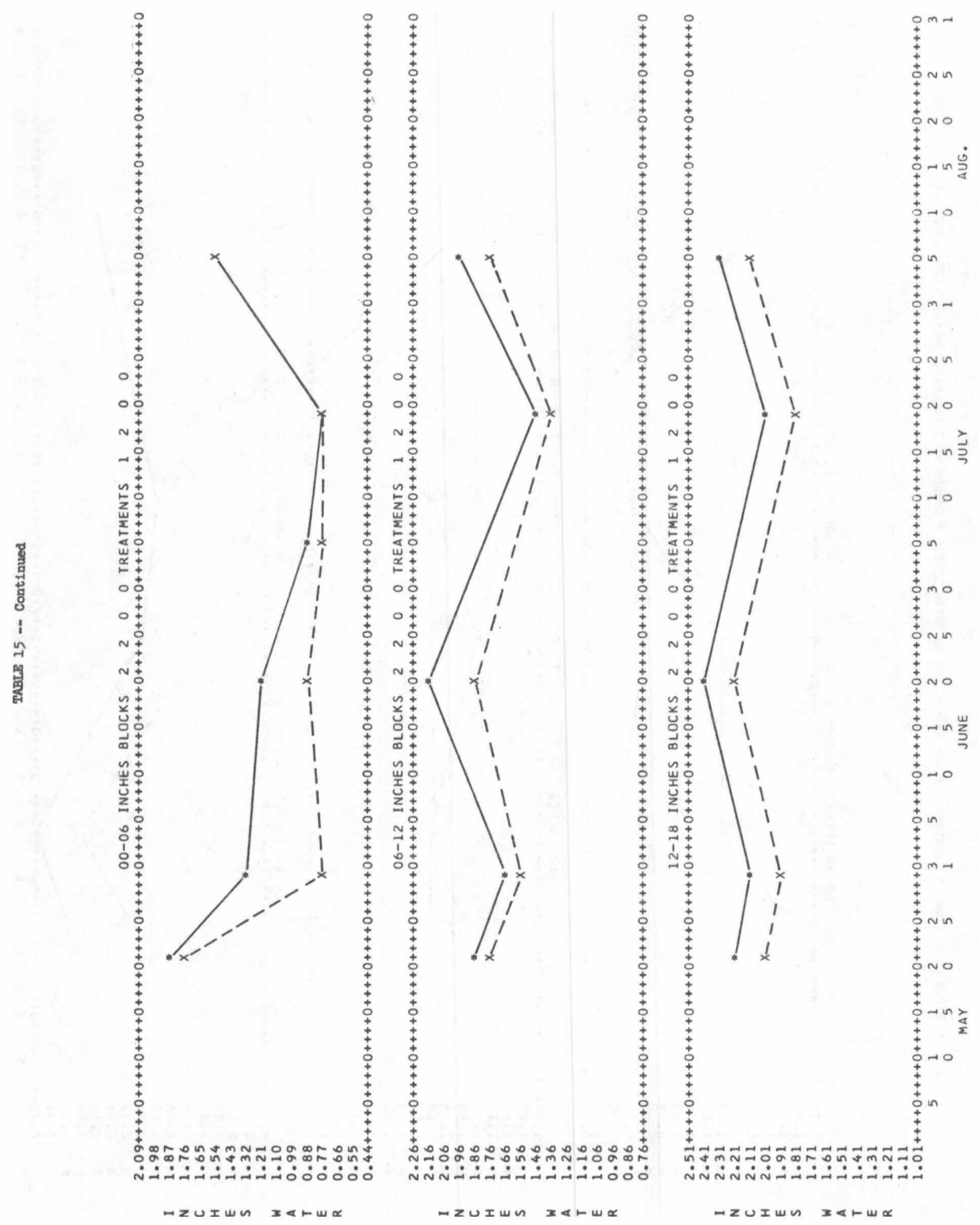


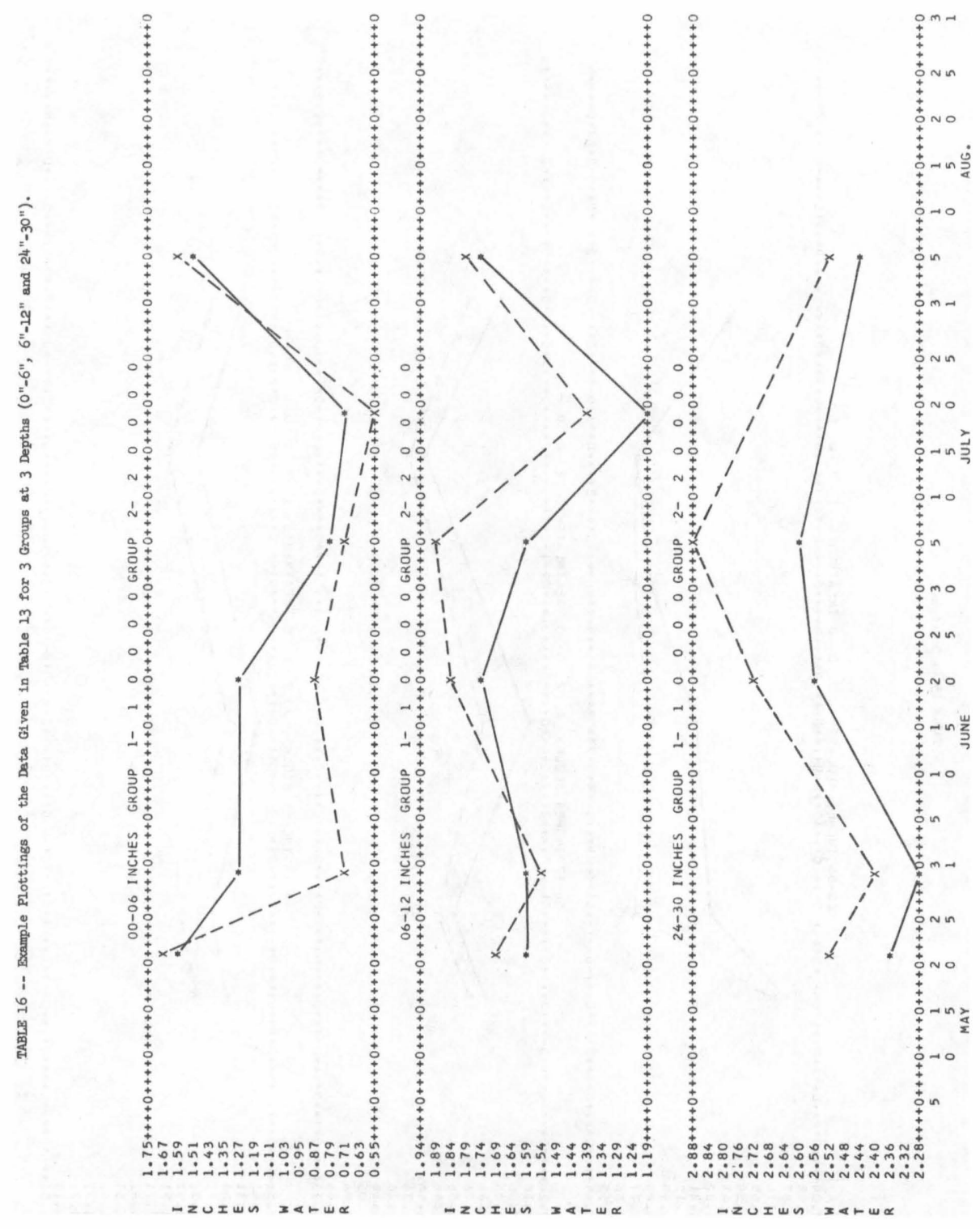



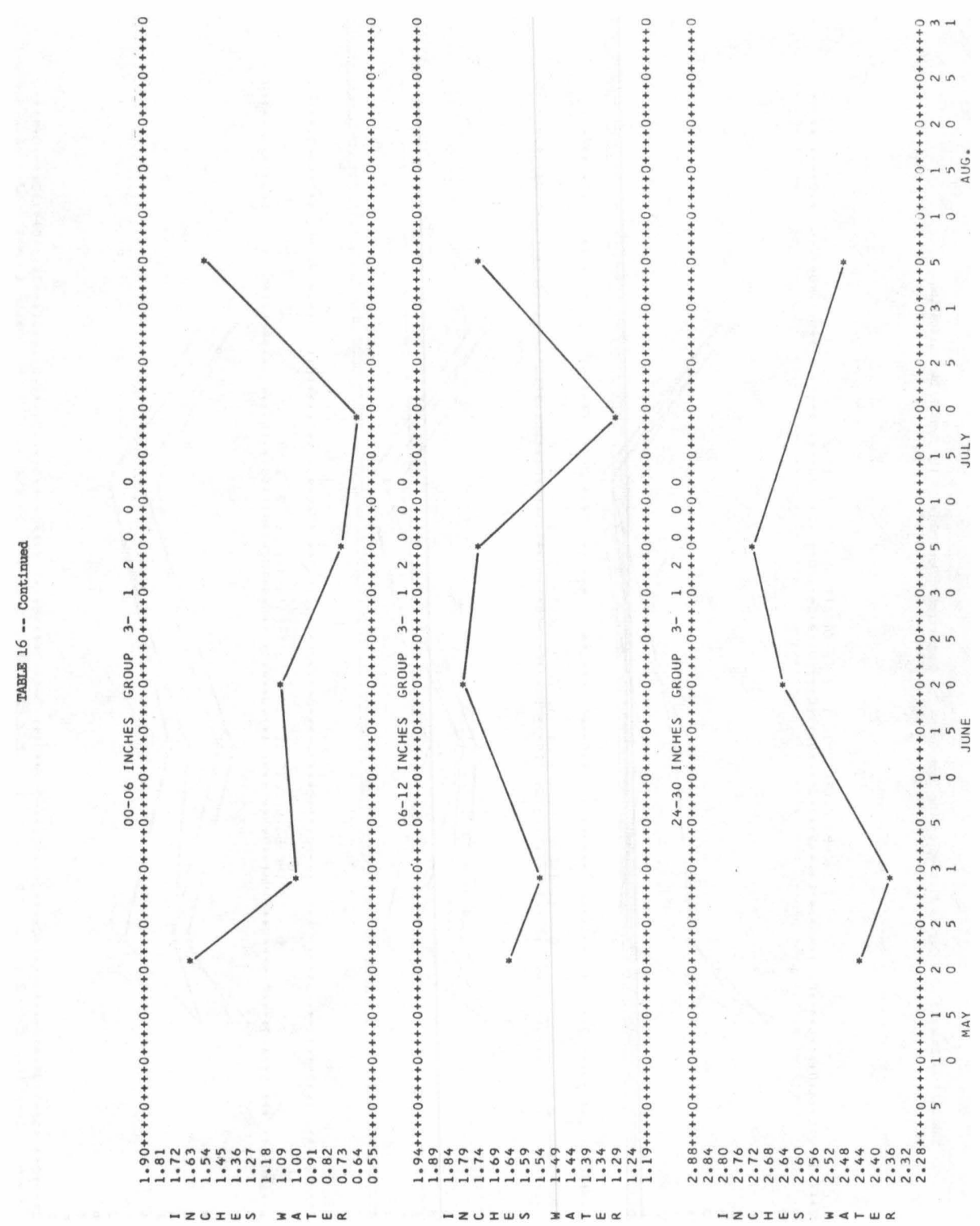


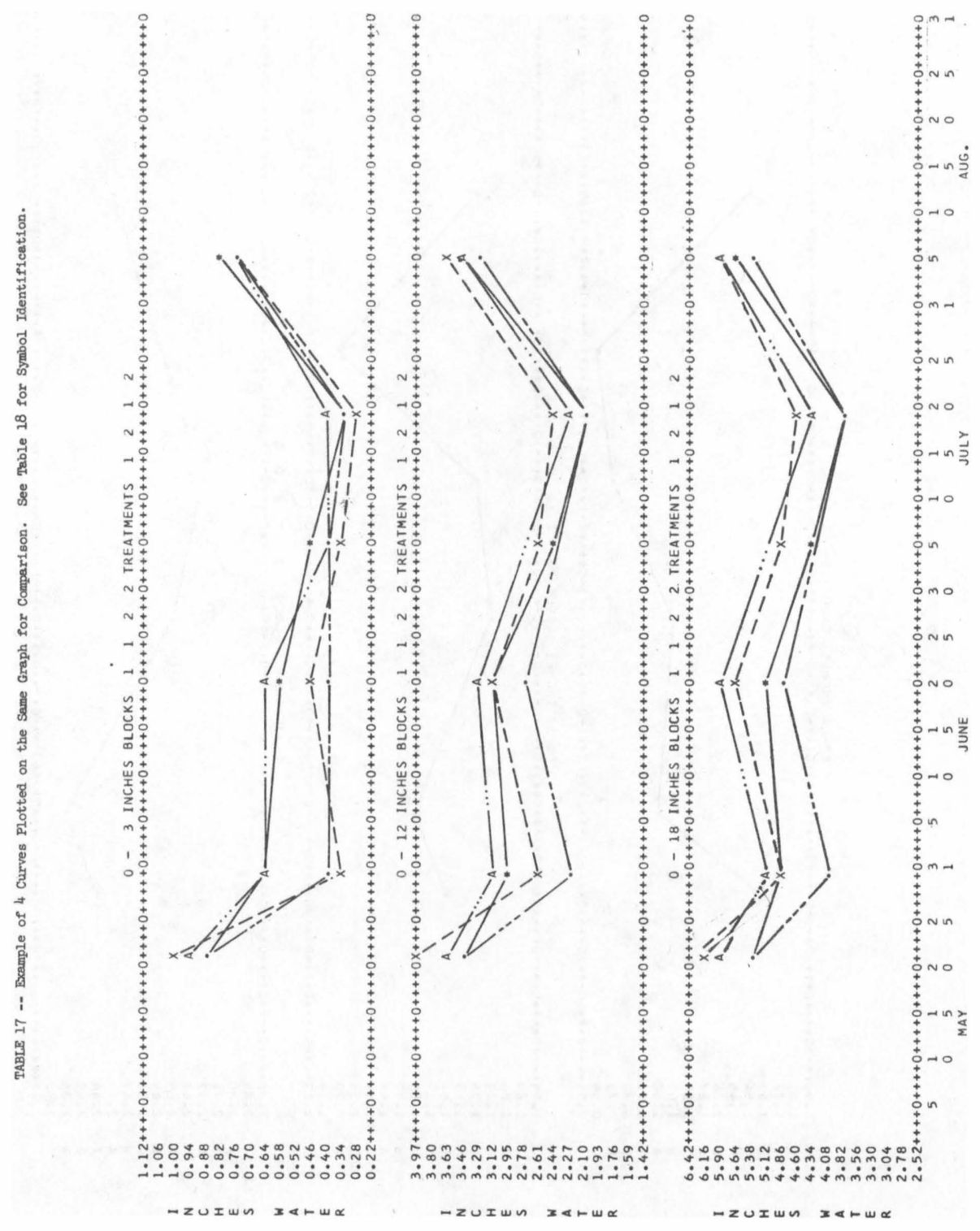


TABLE 18 -- Characters used to Identify Coordinate Points

\begin{tabular}{cc}
\hline Curve No. & Character \\
\hline 1 & $*$ \\
2 & $\mathrm{X}$ \\
3 & $\mathrm{~A}$ \\
4 & $\cdot$ \\
\hline
\end{tabular}

When all curves are plotted, they are in the order of $1,2,3$, and 4 , which means that curve No. 4 takes precedence over curve Nos. 1, 2, and 3. Curve No. 3 takes precedence over curve Nos. 1 and 2 and curve No. 2 takes precedence over curve No. 1. For example, if the total soil moisture at the 6 " depth happens to be 2.00 " for all 4 curves, then the period will be the only character shown on the plotting. Curve Nos. 1, 2, and 3 as well as 4, will go through this one point.

\section{STATISTICAL ANALYSIS}

Section 2 was designed so that the output would be applicable for use in Section 3 (Appendix A4). The variables that have been coded for this analysis are blocks, dates, depths, experimental replications, and treatments. Any combination of these variables may be used in the statistical analysis by proper coding. The output cards are coded for efficient sorting by an IBM sorter. Sequential codes for each variable are contained in the following card columns: (a) depths - columns 1-2, (b) treatments - columns 3-4, (c) blocks - columns 5-6, (d) dates - columns 7-8, and (e) experimental replications - columns 9-10. To arrange the data in the correct order as input for Section 3, the data are sorted according to depths, treatments, blocks, dates, and experimental replications. 
APPENDIX A

Reduction of Basic Field Moisture

and

Analysis of Variance for Factorial Design 


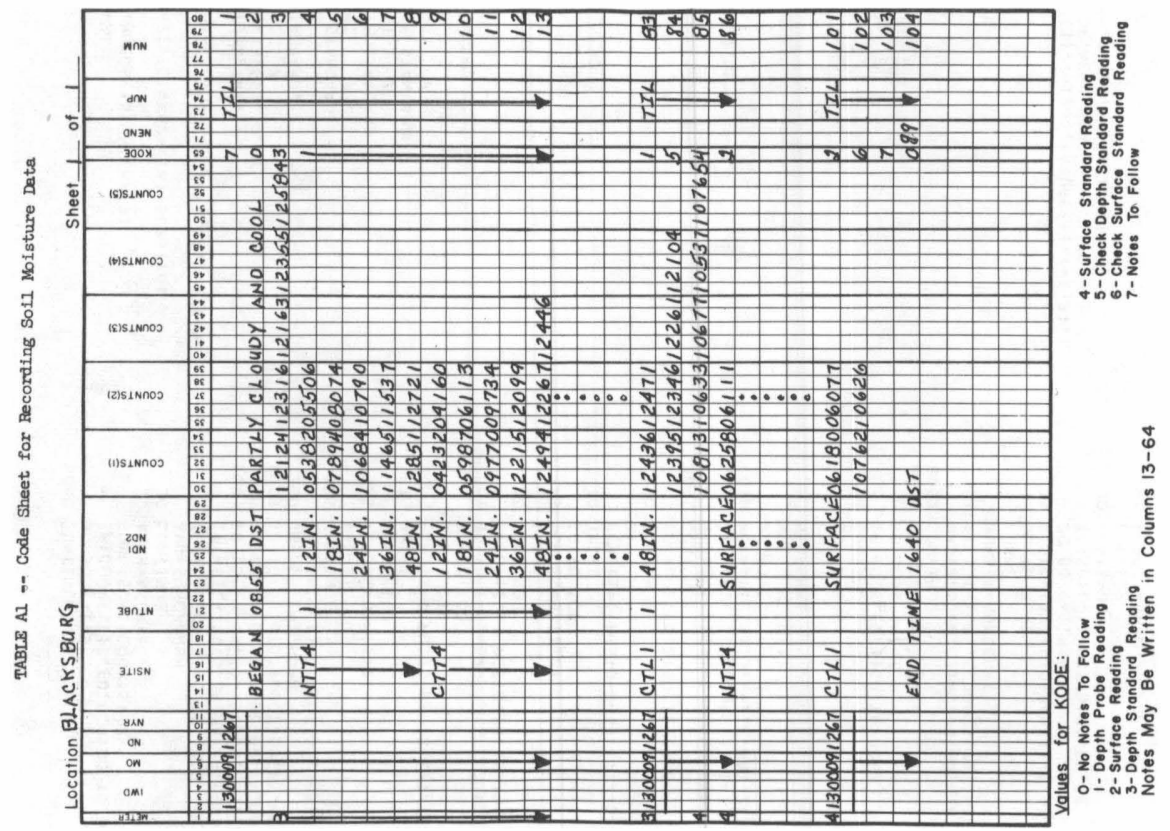




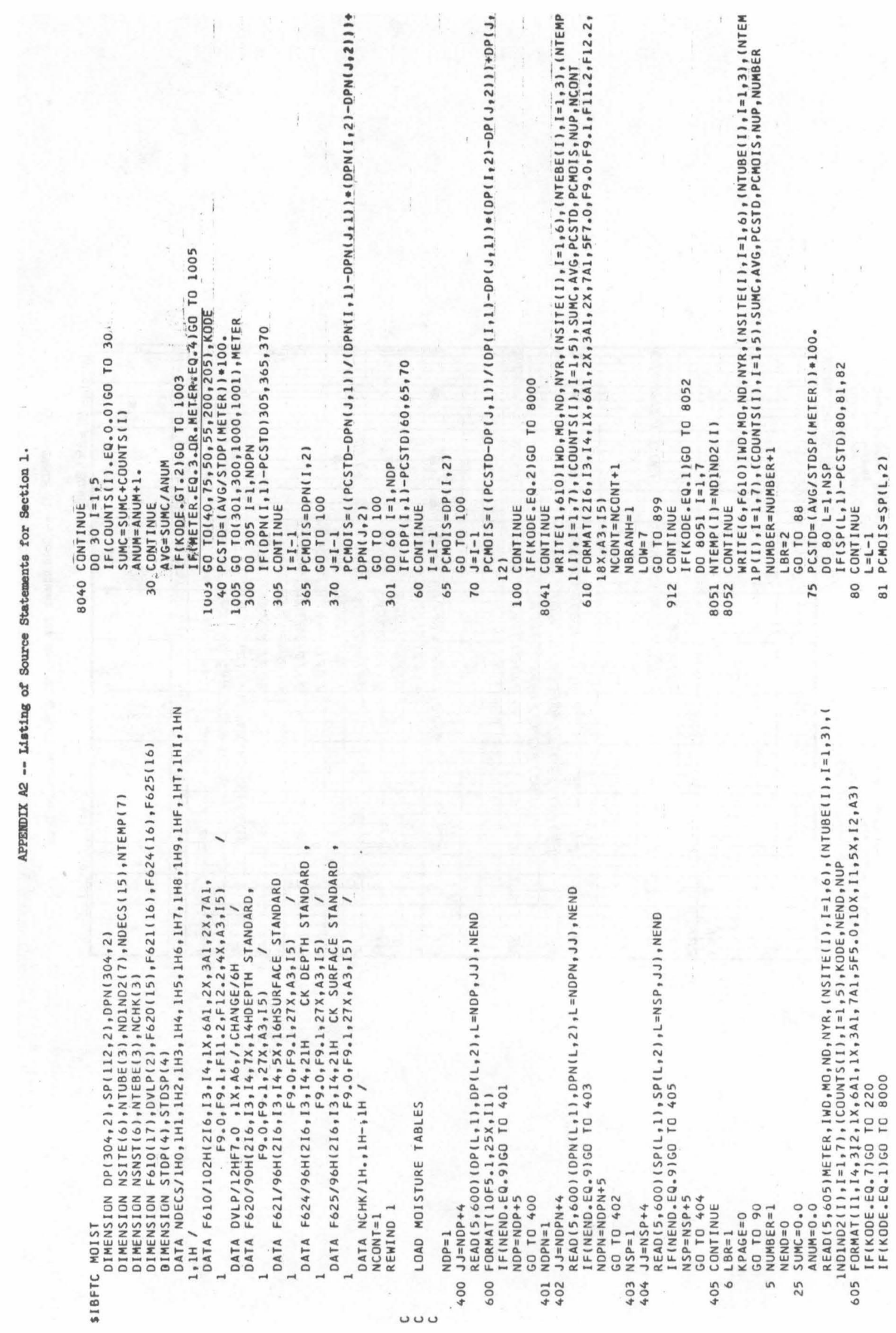




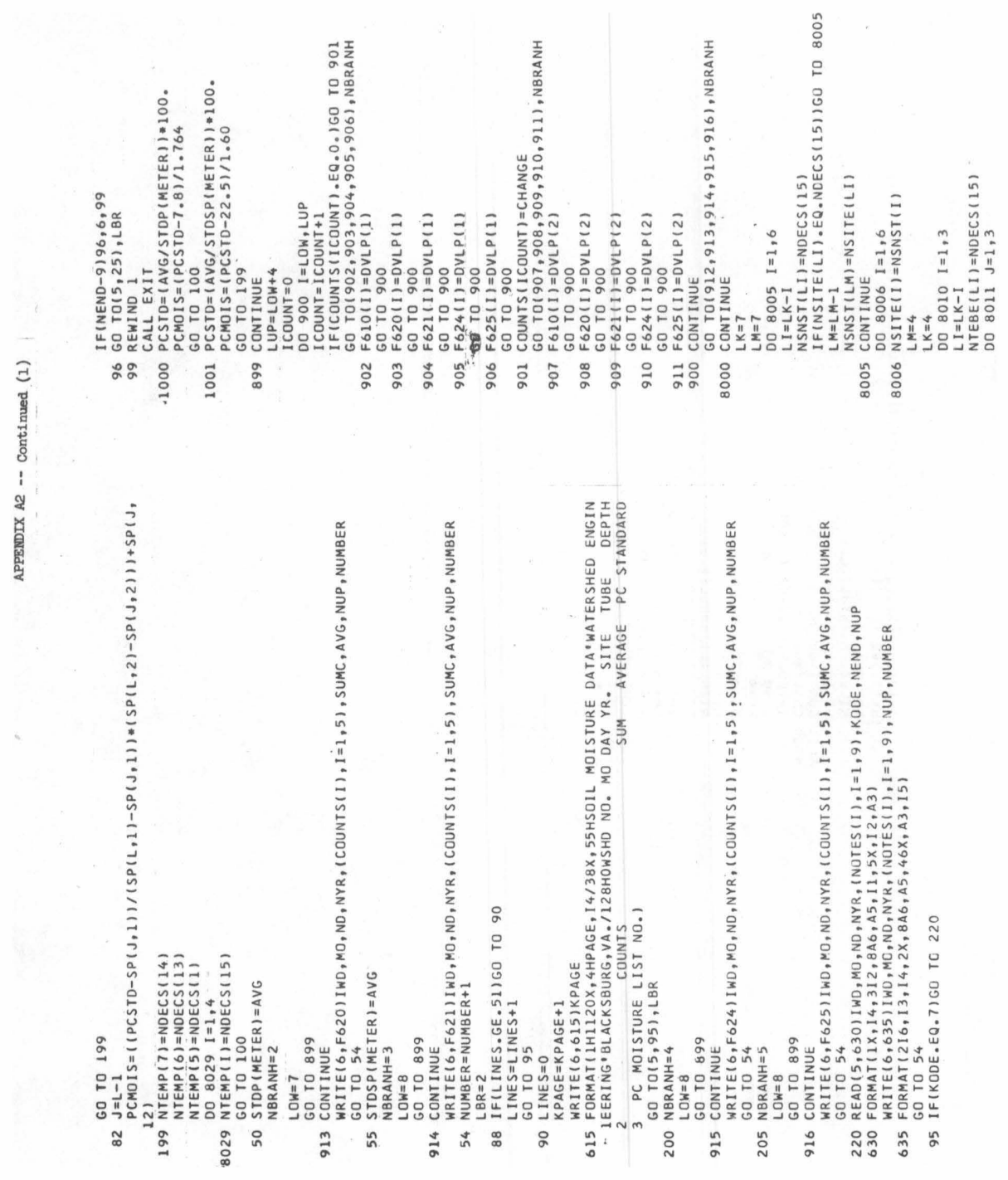




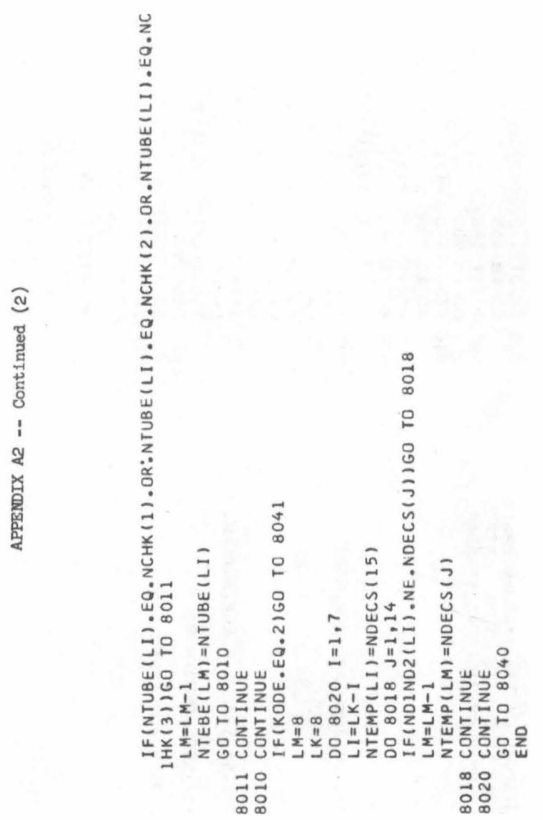




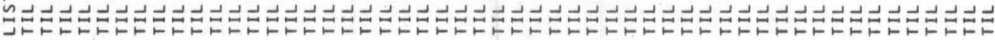

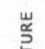

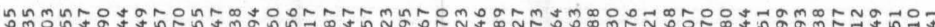

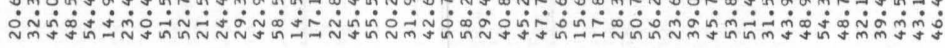

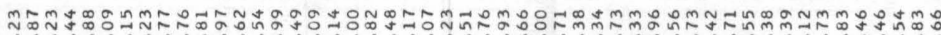

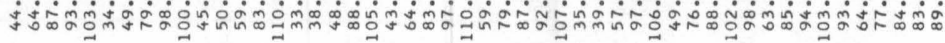

4 to

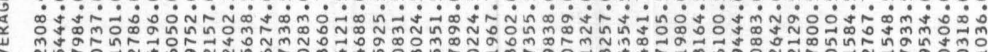

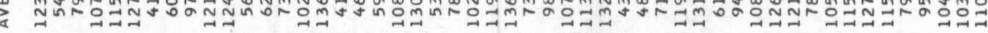

₹

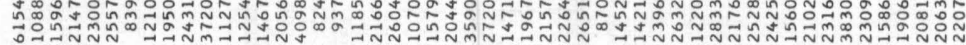

空

䓛

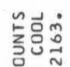

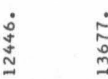

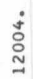

$\underset{\dot{a}}{\dot{a}}$

$\underset{\sim}{\sim}$

$\stackrel{\dot{\overrightarrow{0}}}{\stackrel{\sim}{5}}$

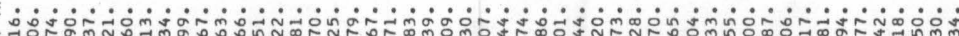

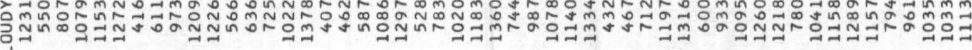
3

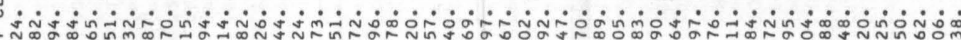

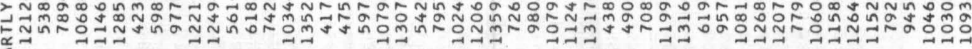
여의

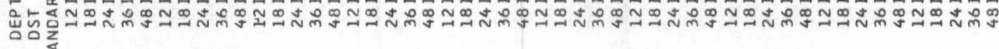

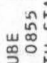
z⿺辶巛ّ⿵人一

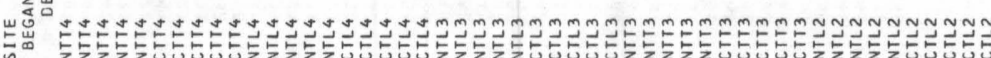
غ่5555555555555555555555555555555555555555555555555555

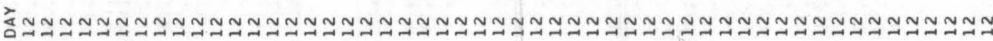

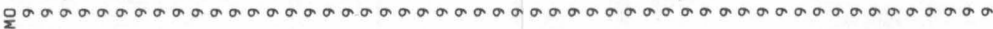
$\dot{2}$

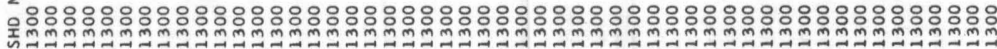


N

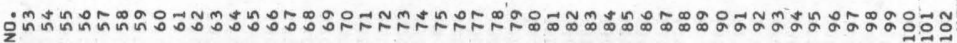

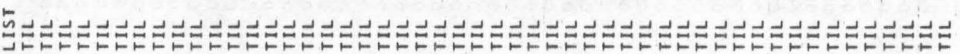
峁

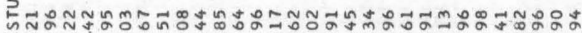

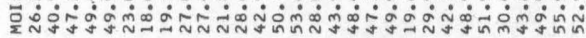

ت

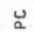

(1)

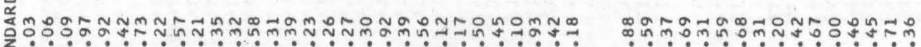

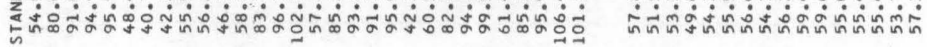

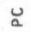

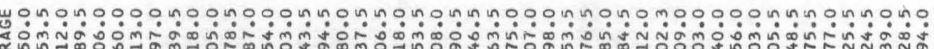

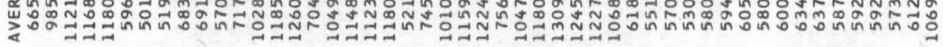

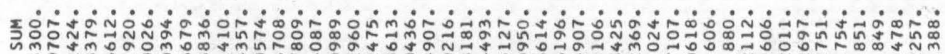

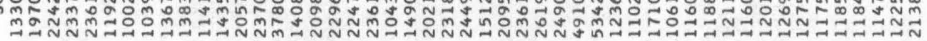

产

$\stackrel{\substack{\infty \\ \infty}}{\stackrel{\infty}{\infty}}$

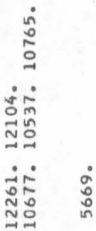

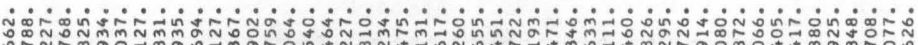
:

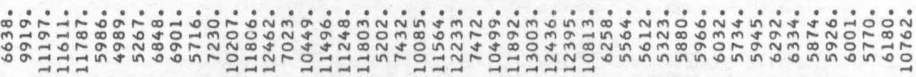

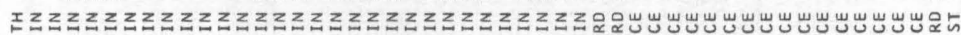

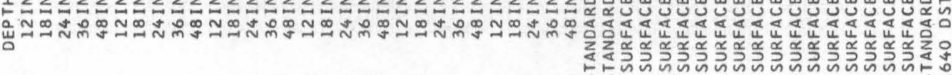

宸

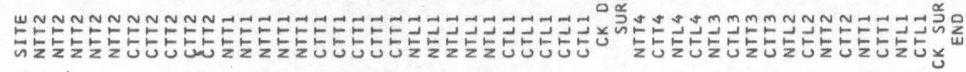

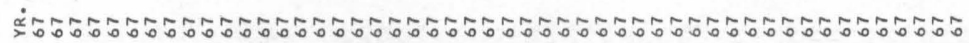

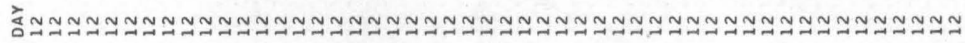

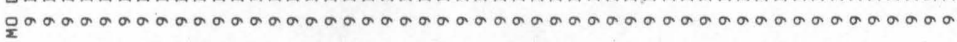
$\dot{2}$

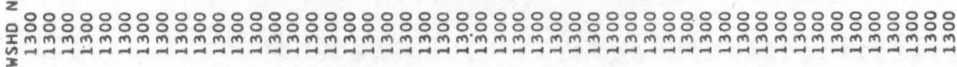




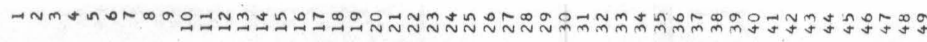

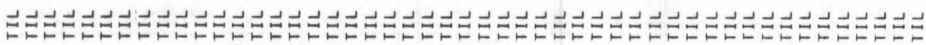

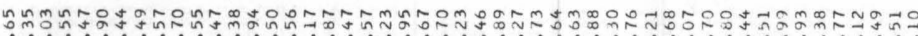

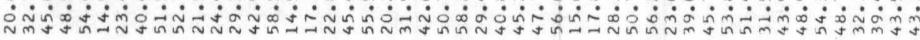

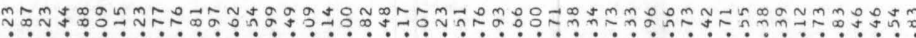

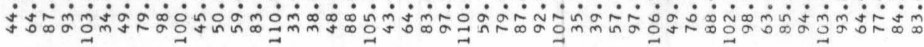

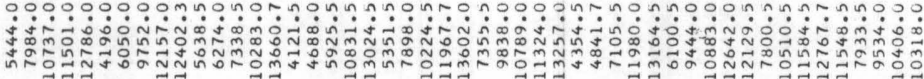

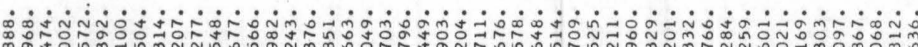

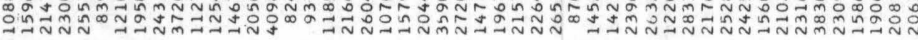

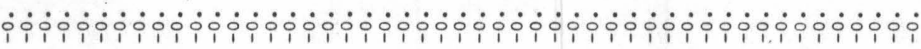

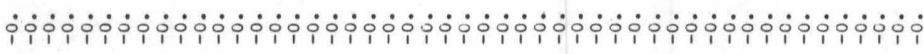

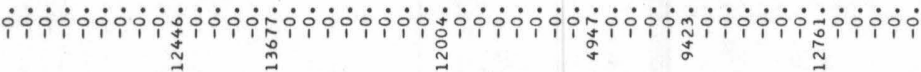
ดिं

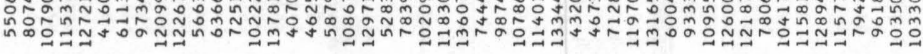

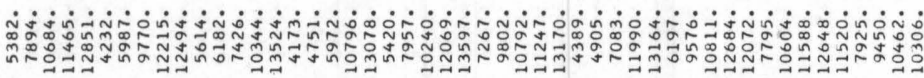

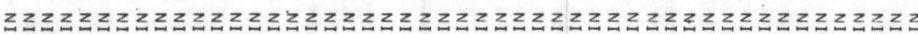

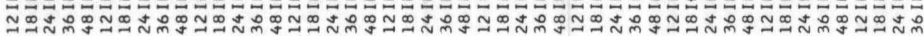

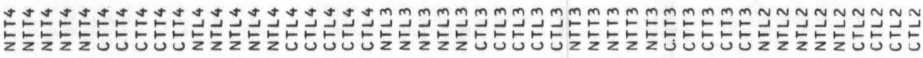
50505050505505505505050505050505050505050505050505

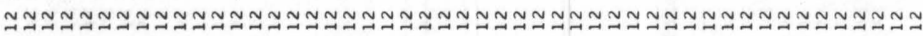

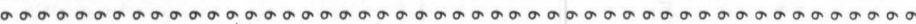

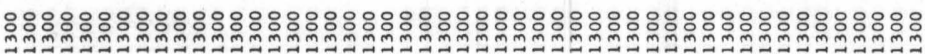




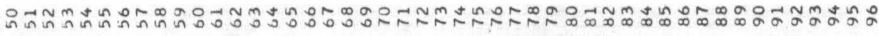

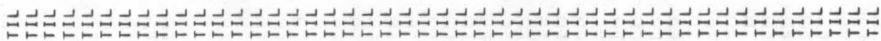

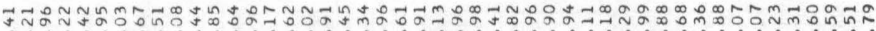

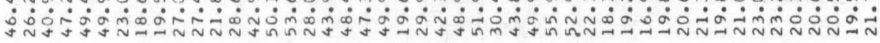

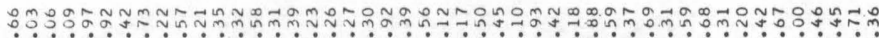

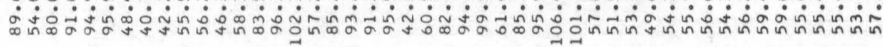

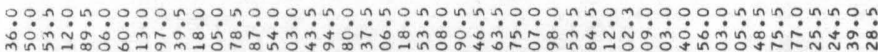

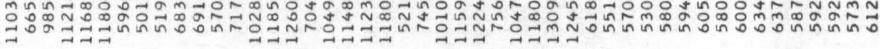

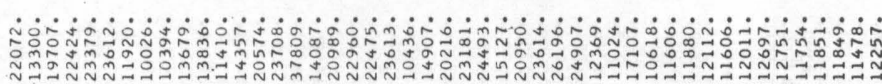

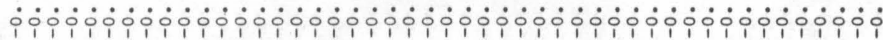
(1)

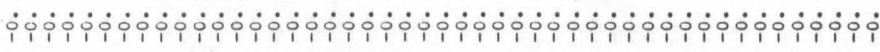

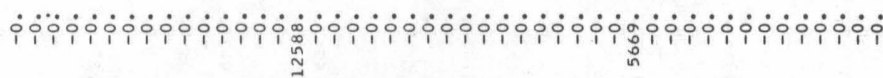
में

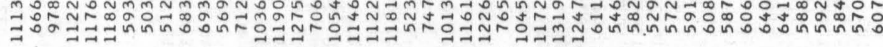

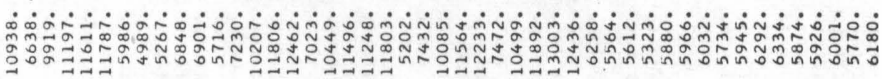

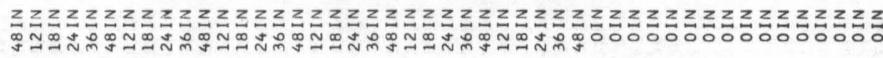

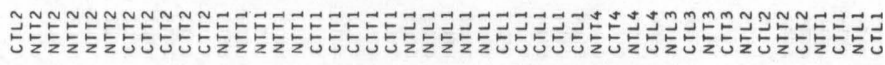

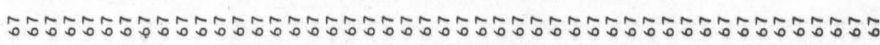

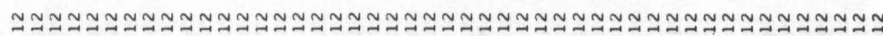

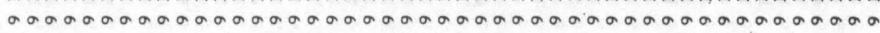

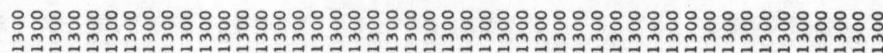




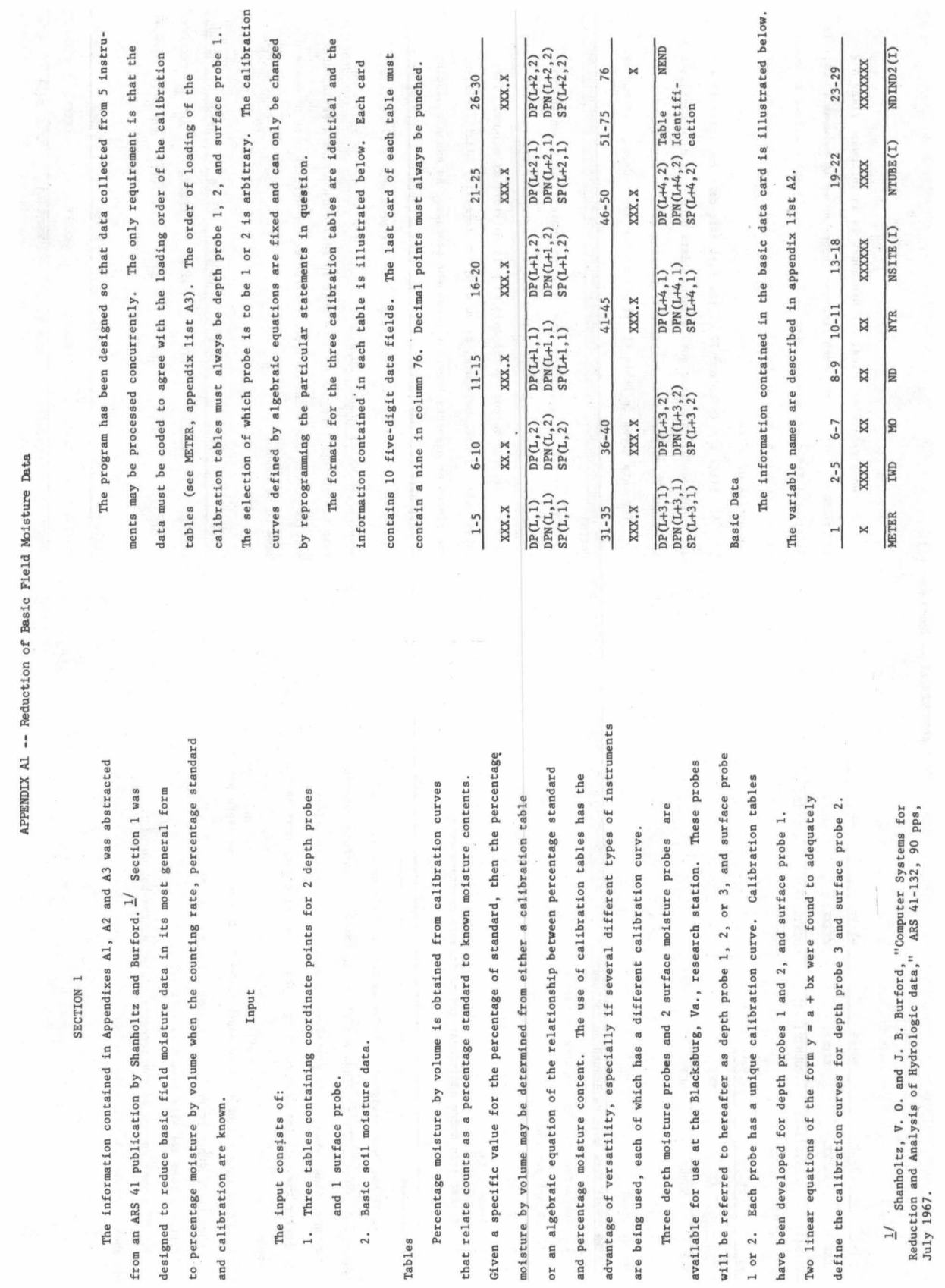




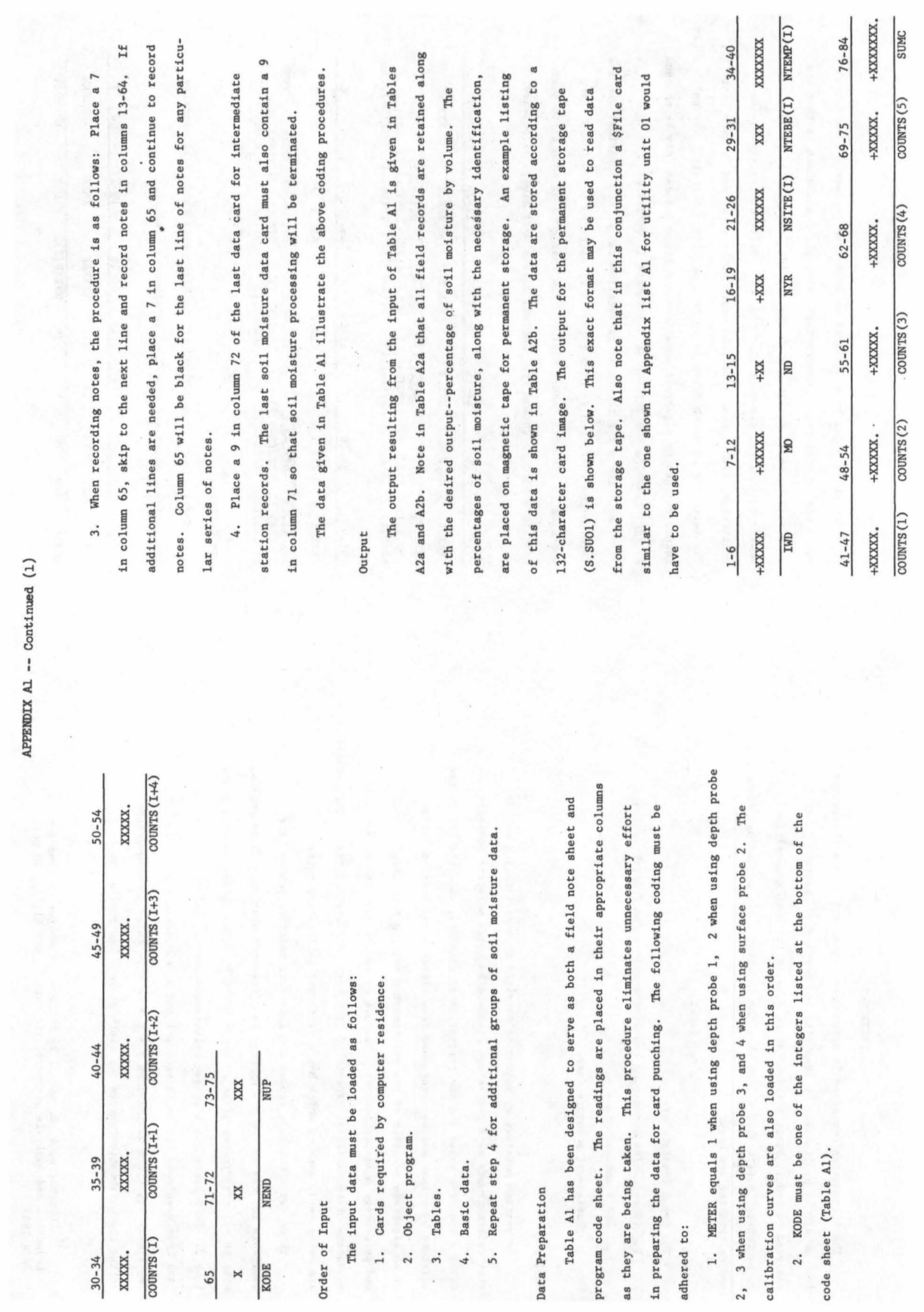




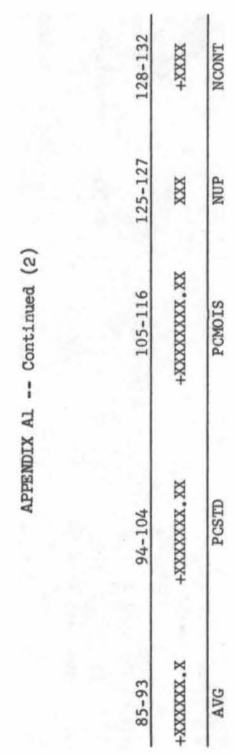




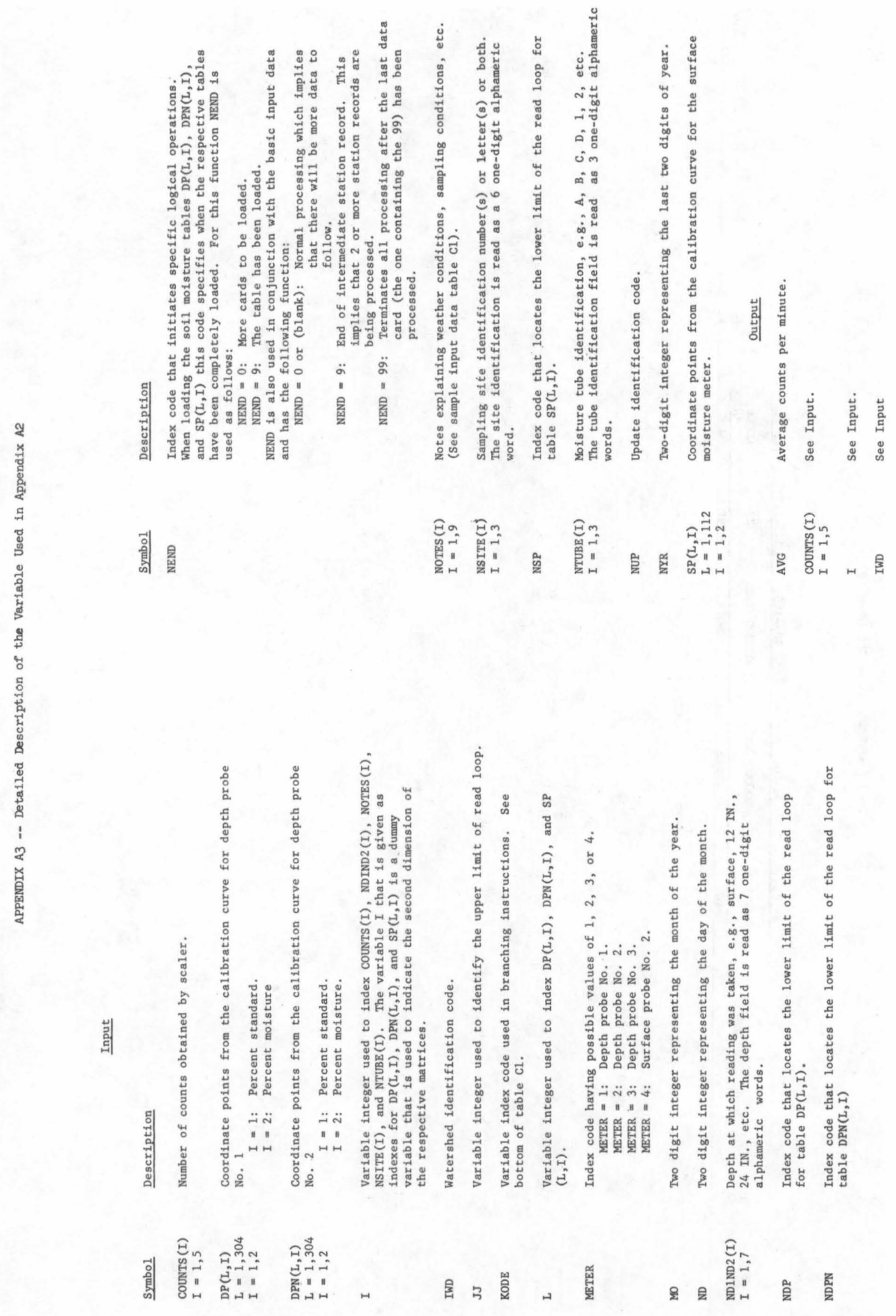




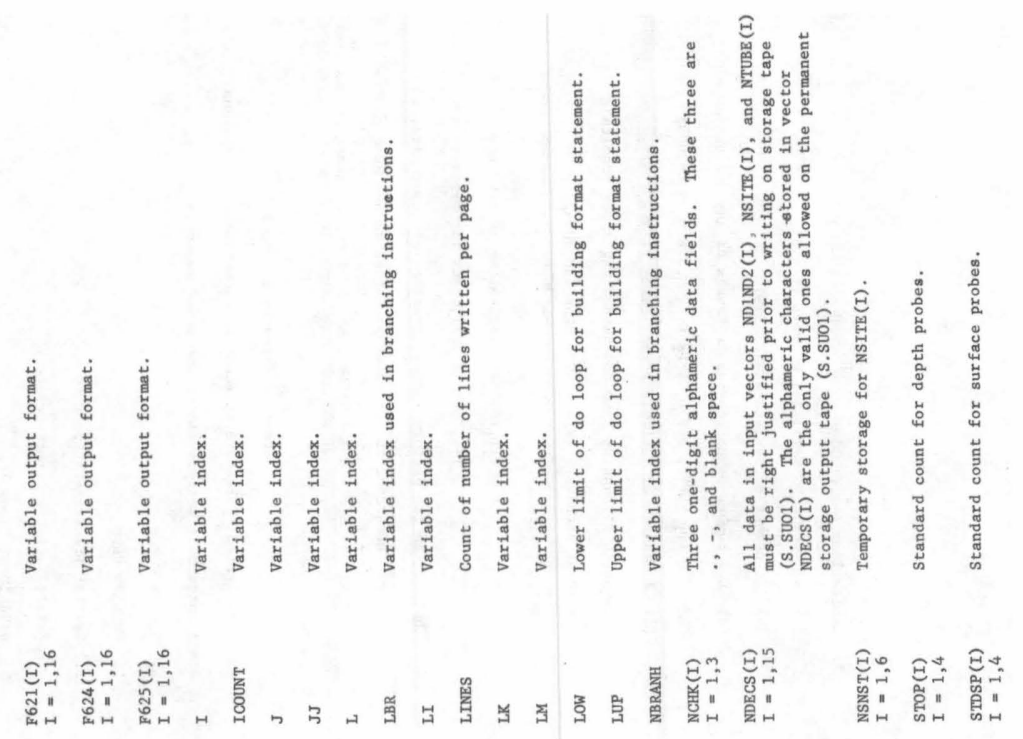

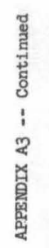

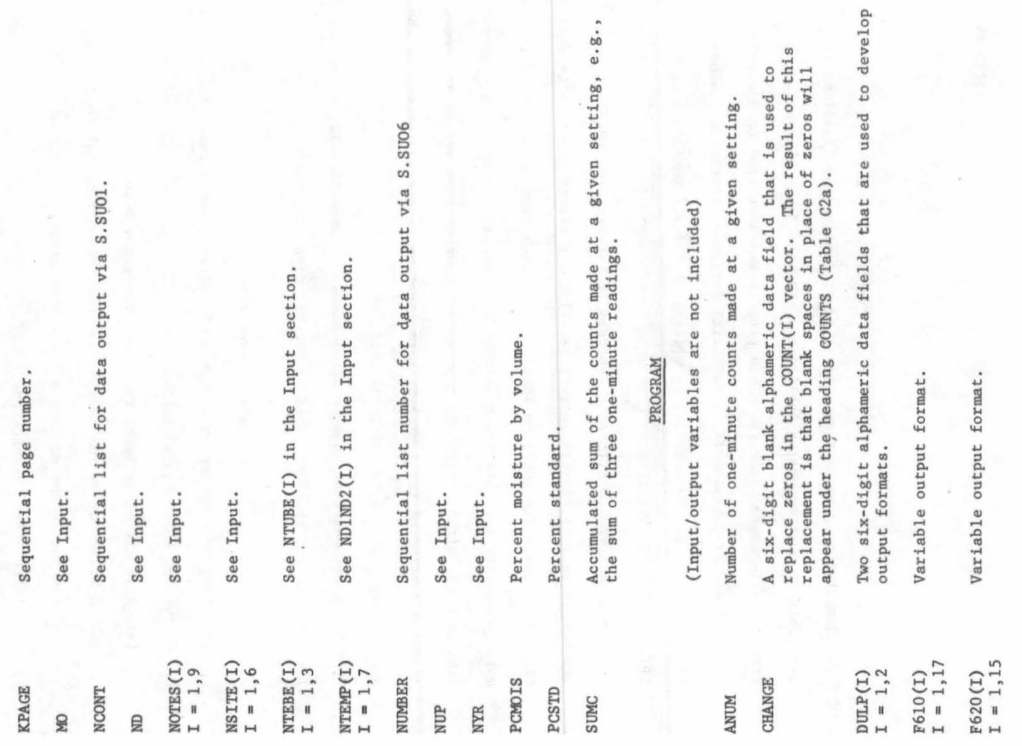




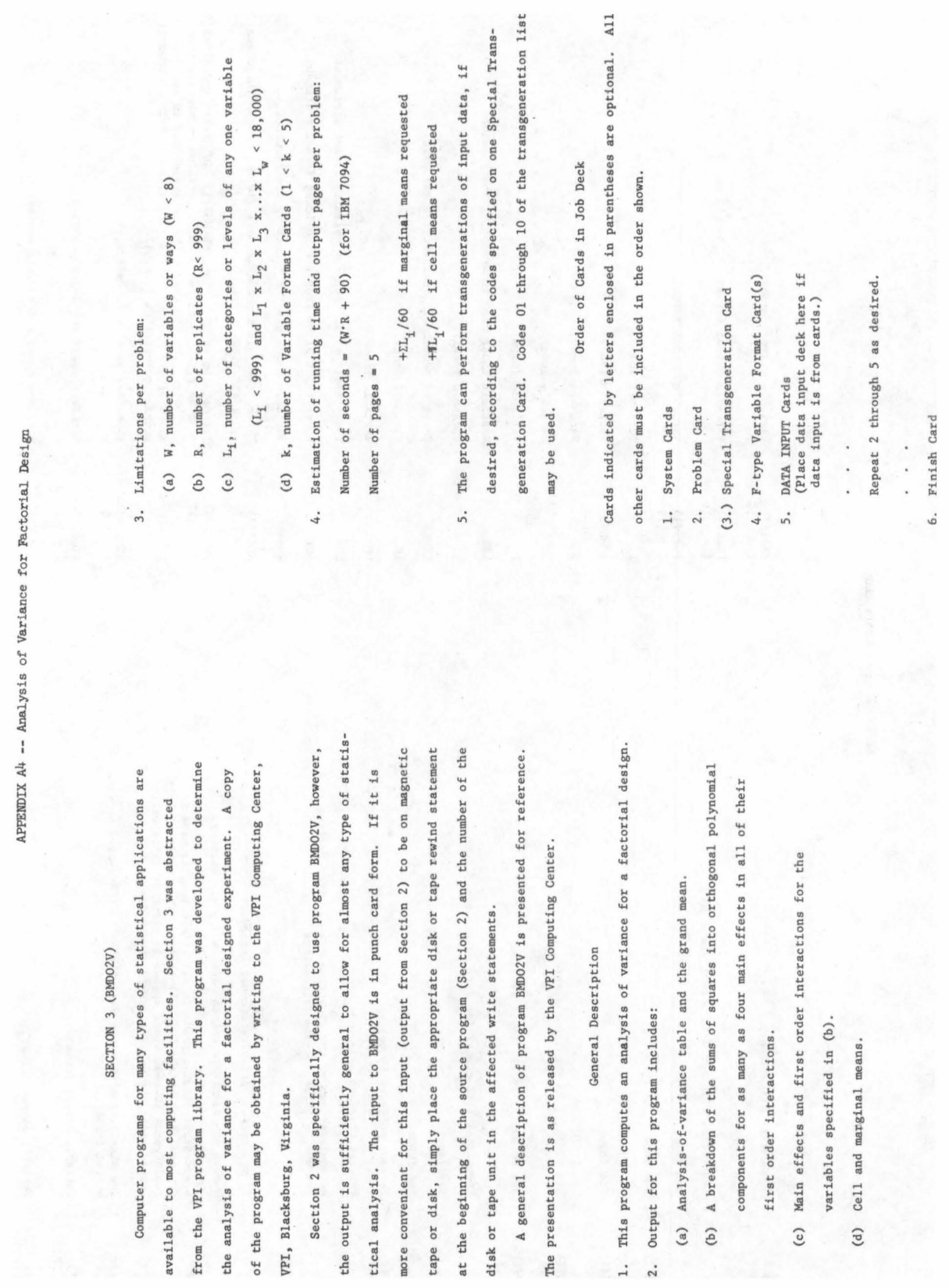




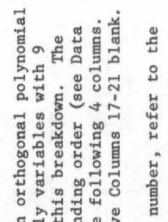

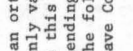

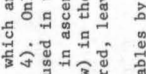

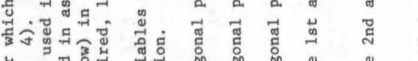

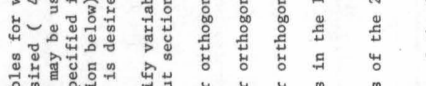

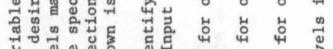

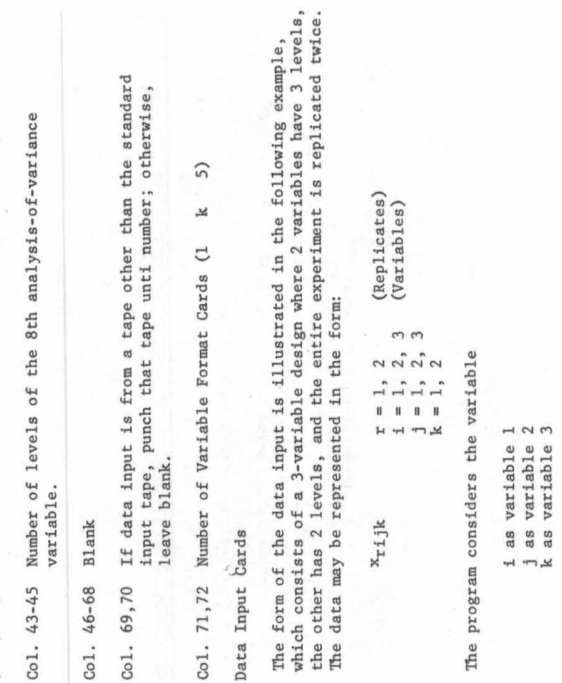

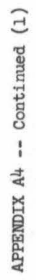

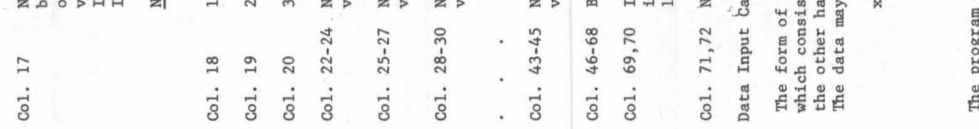

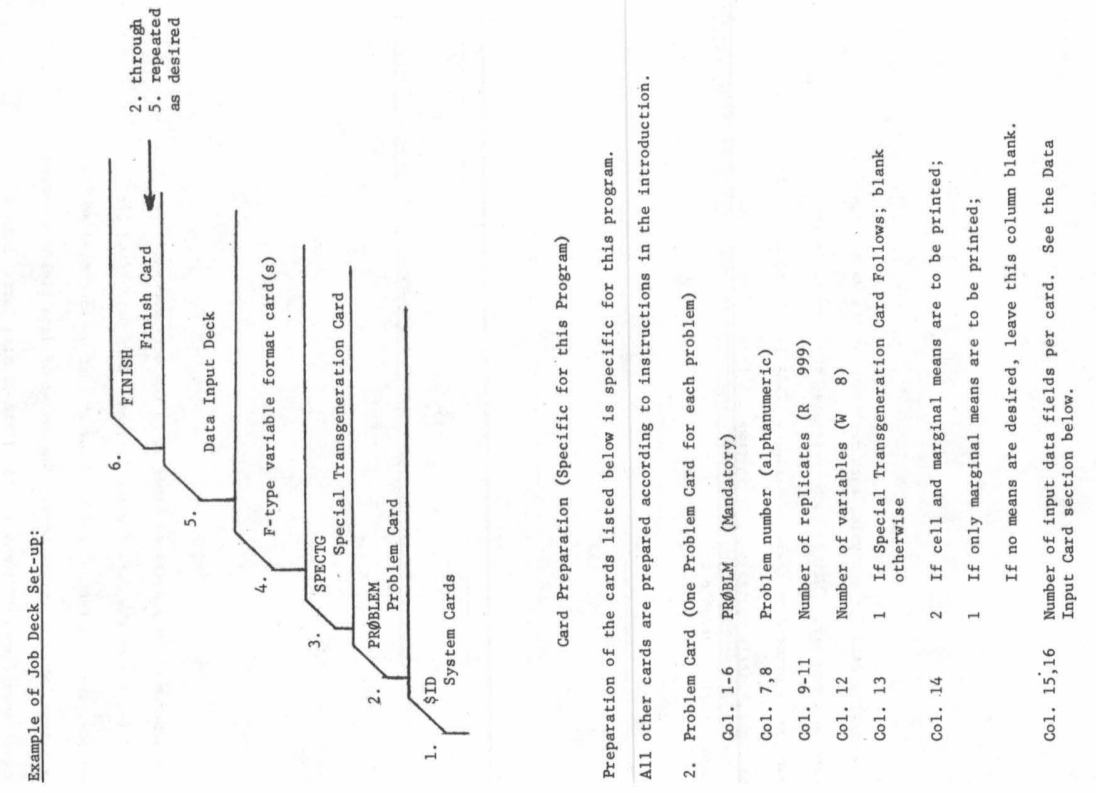




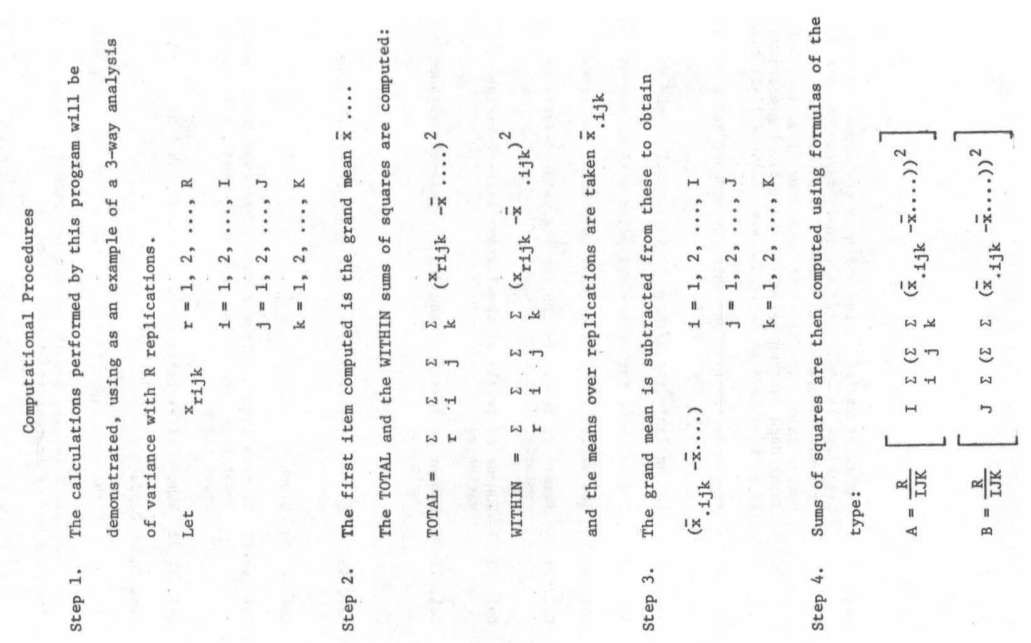

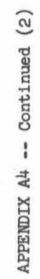

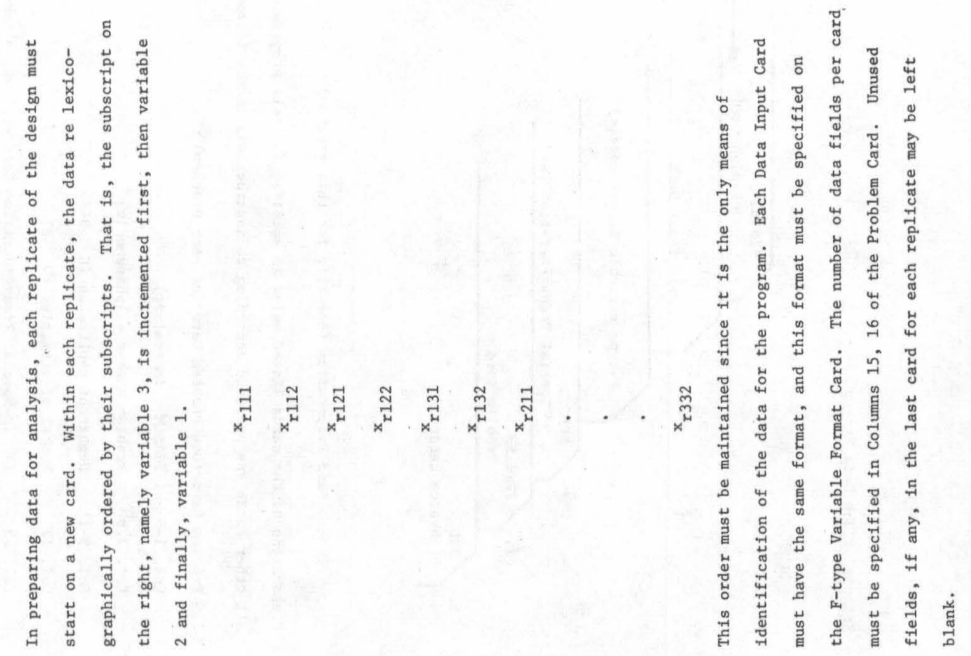




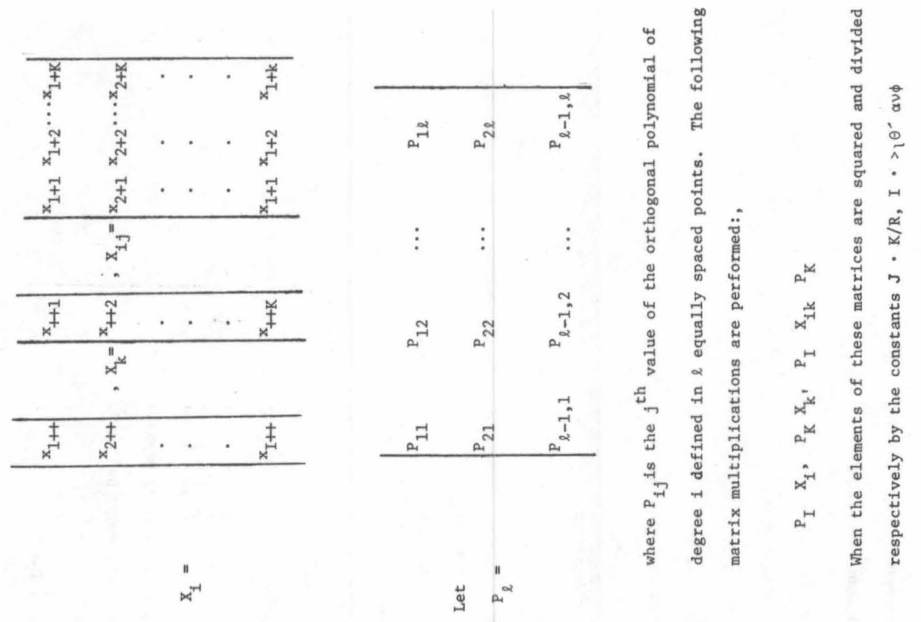

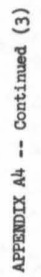

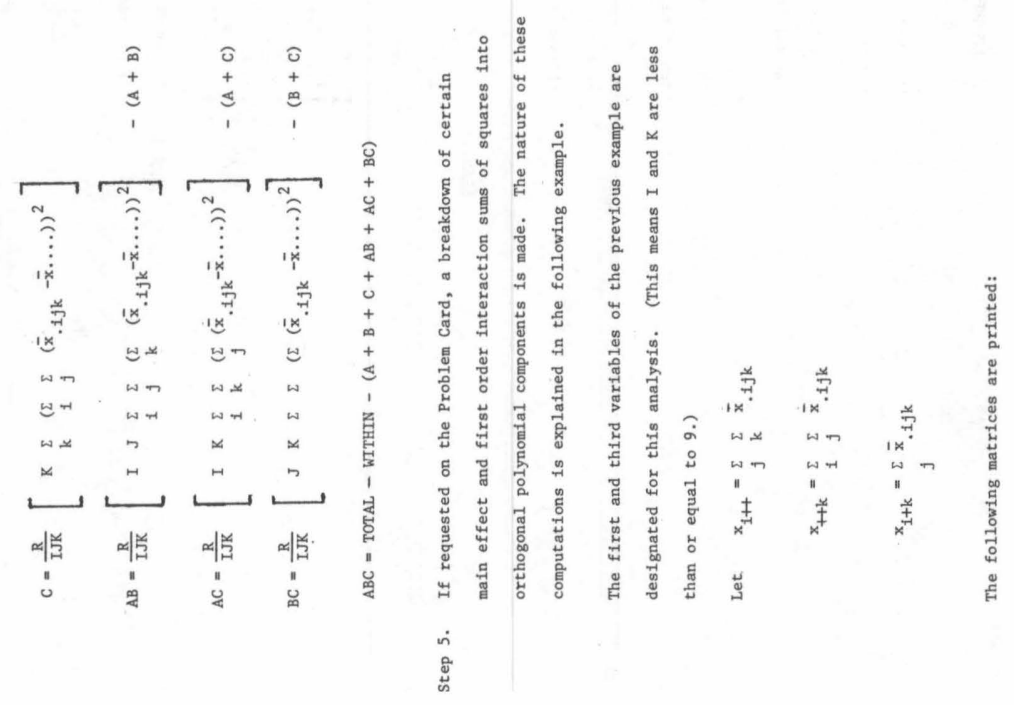




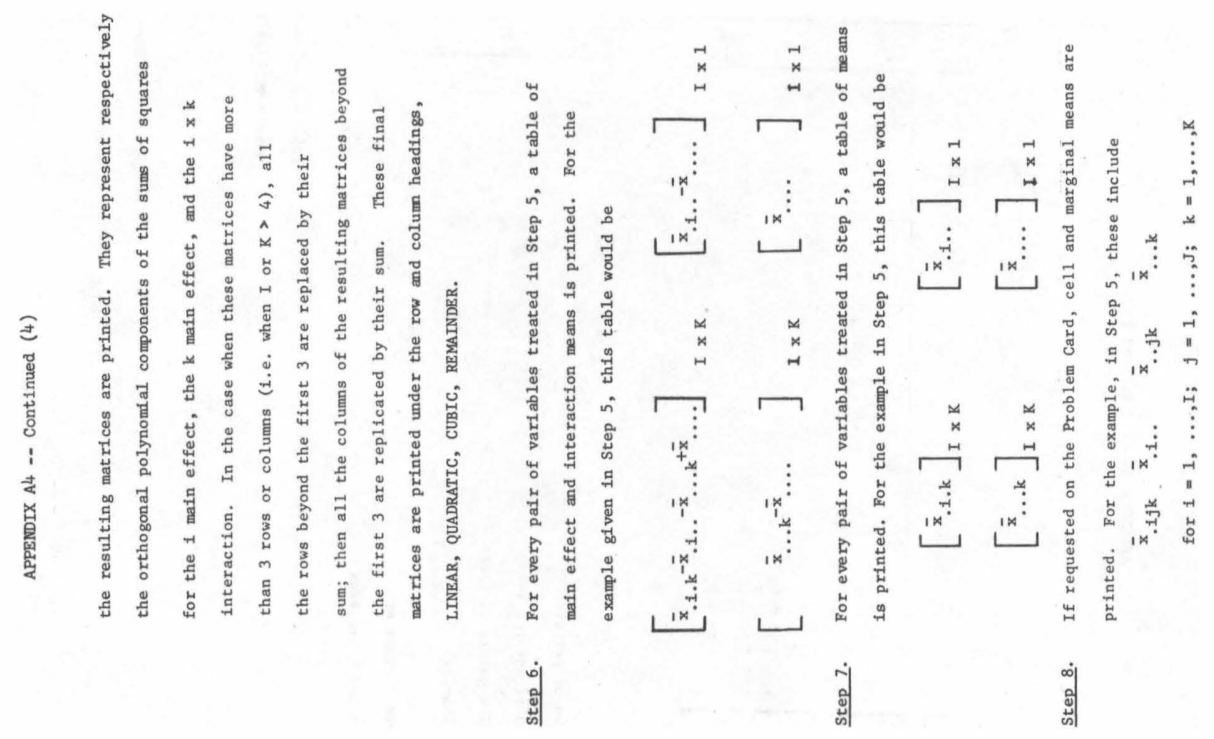


APPENDIX B

Program Listing and Descriptions

of Variables for Soil Moisture

Reduction -- Section II 


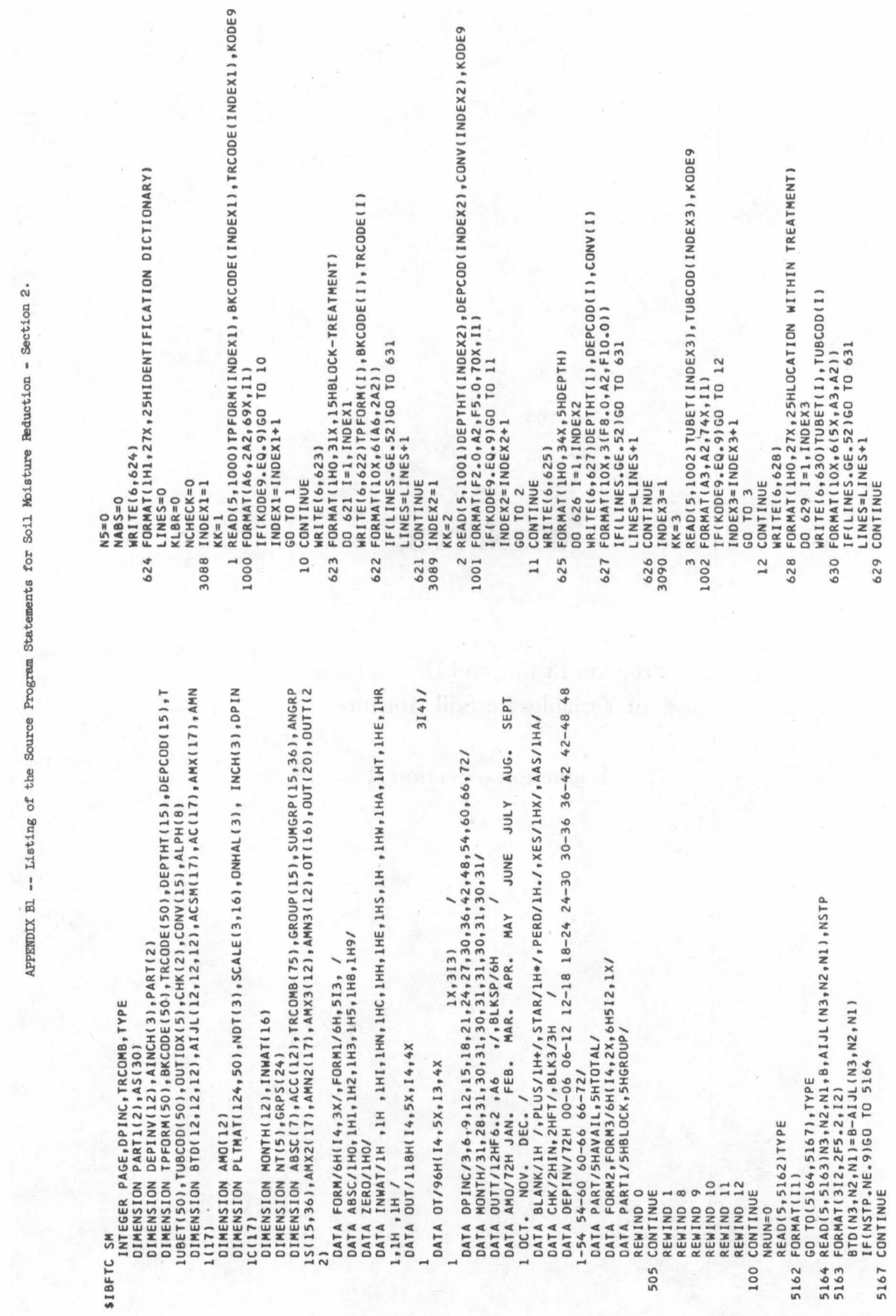




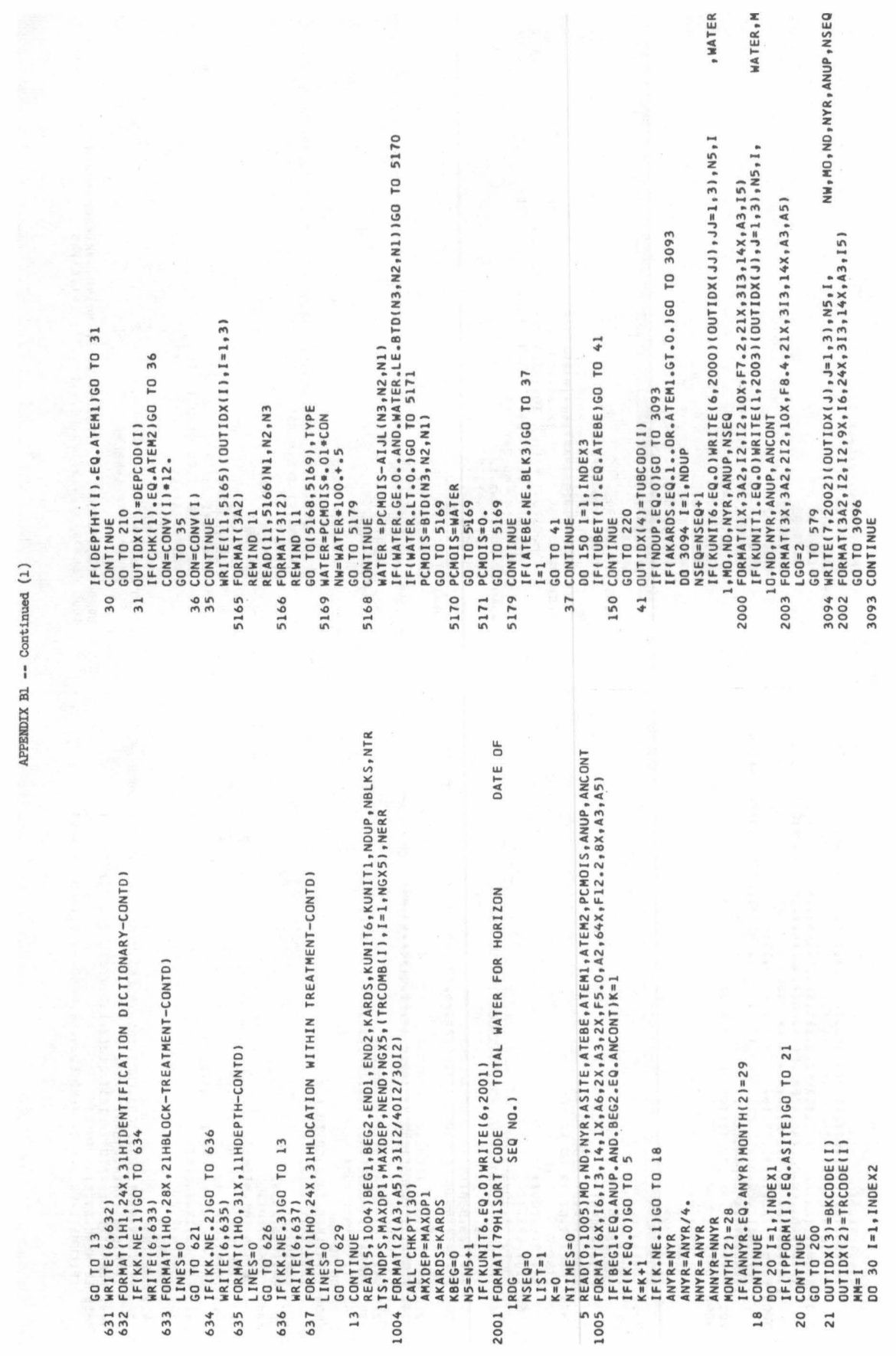



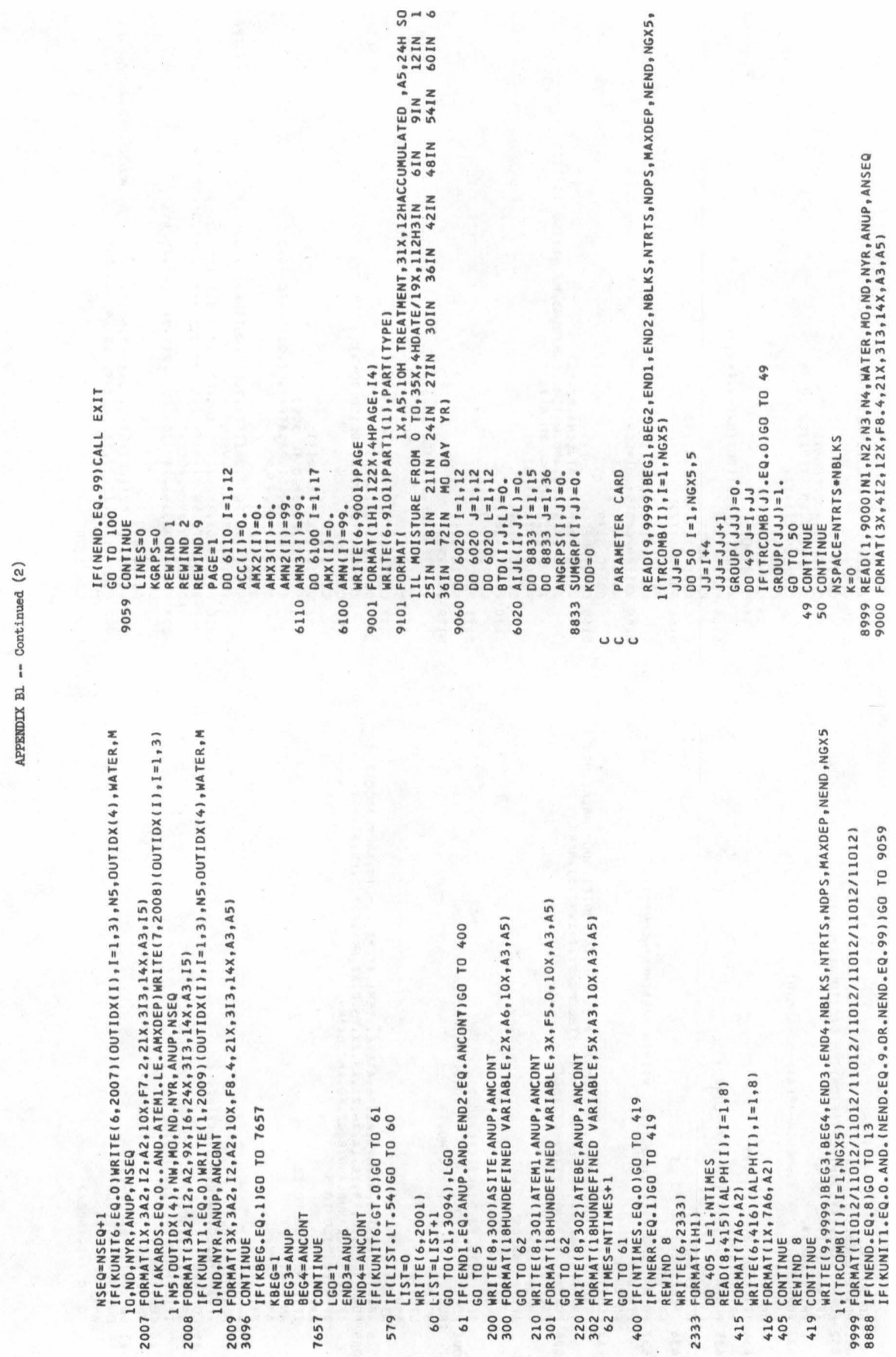


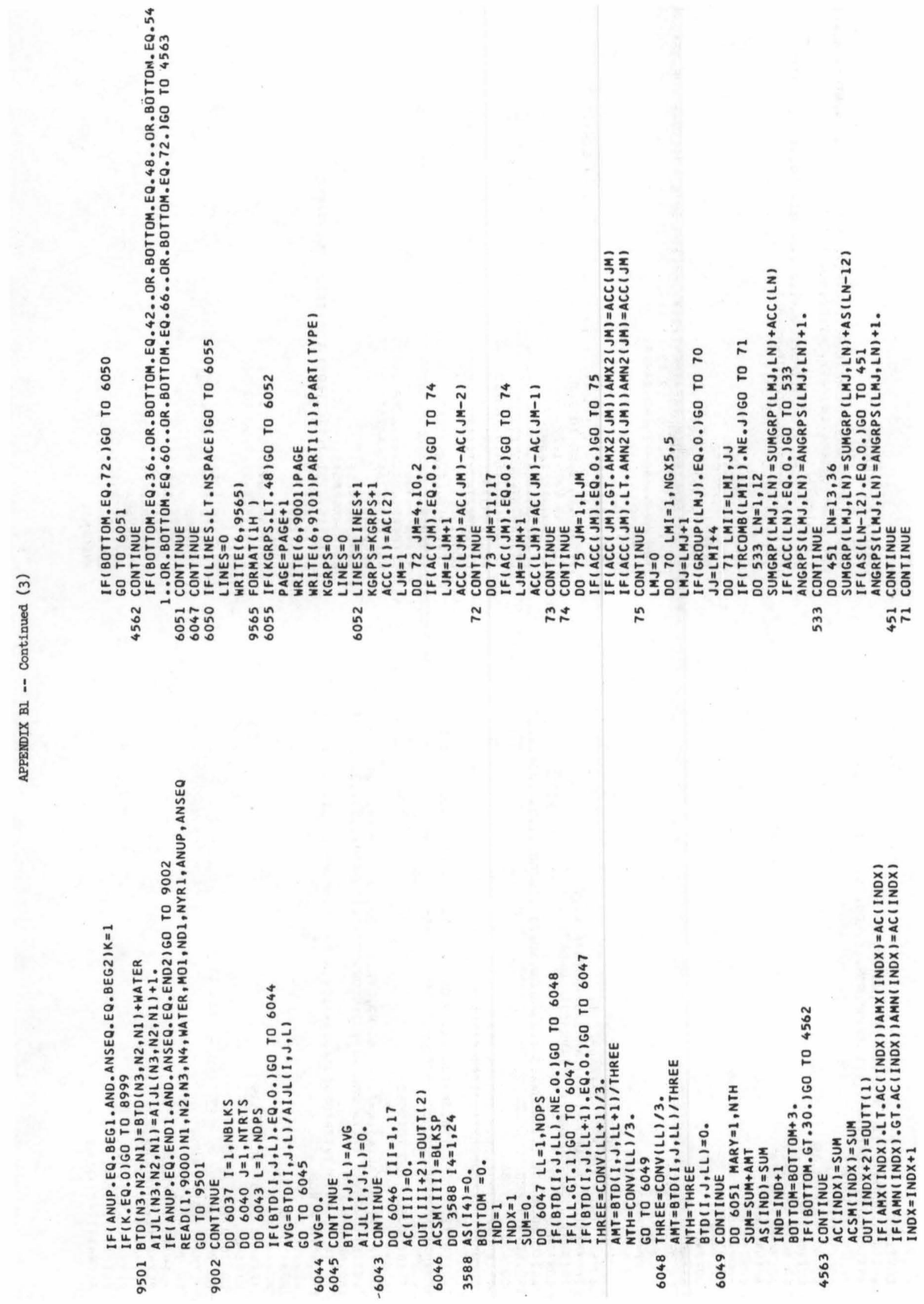




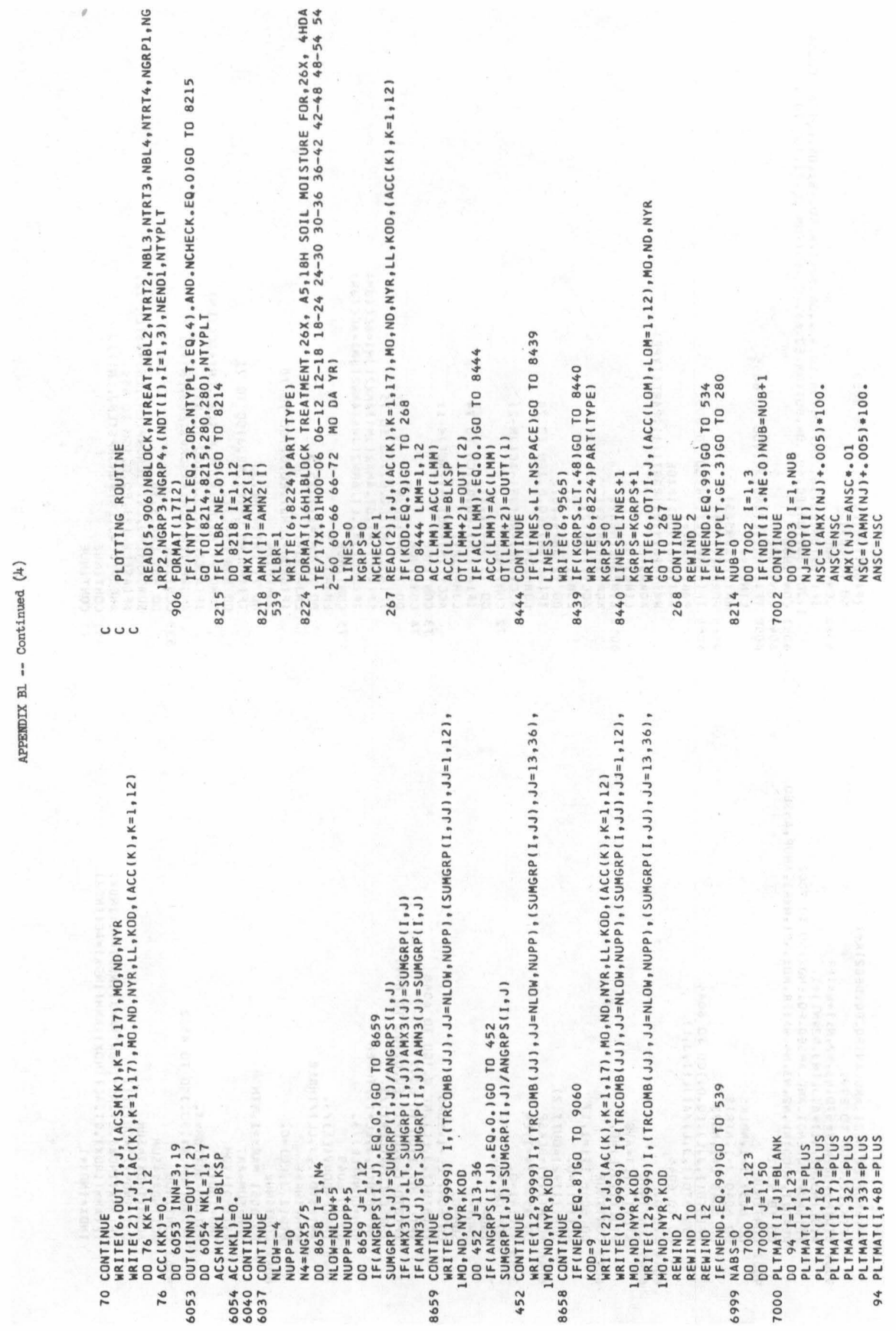




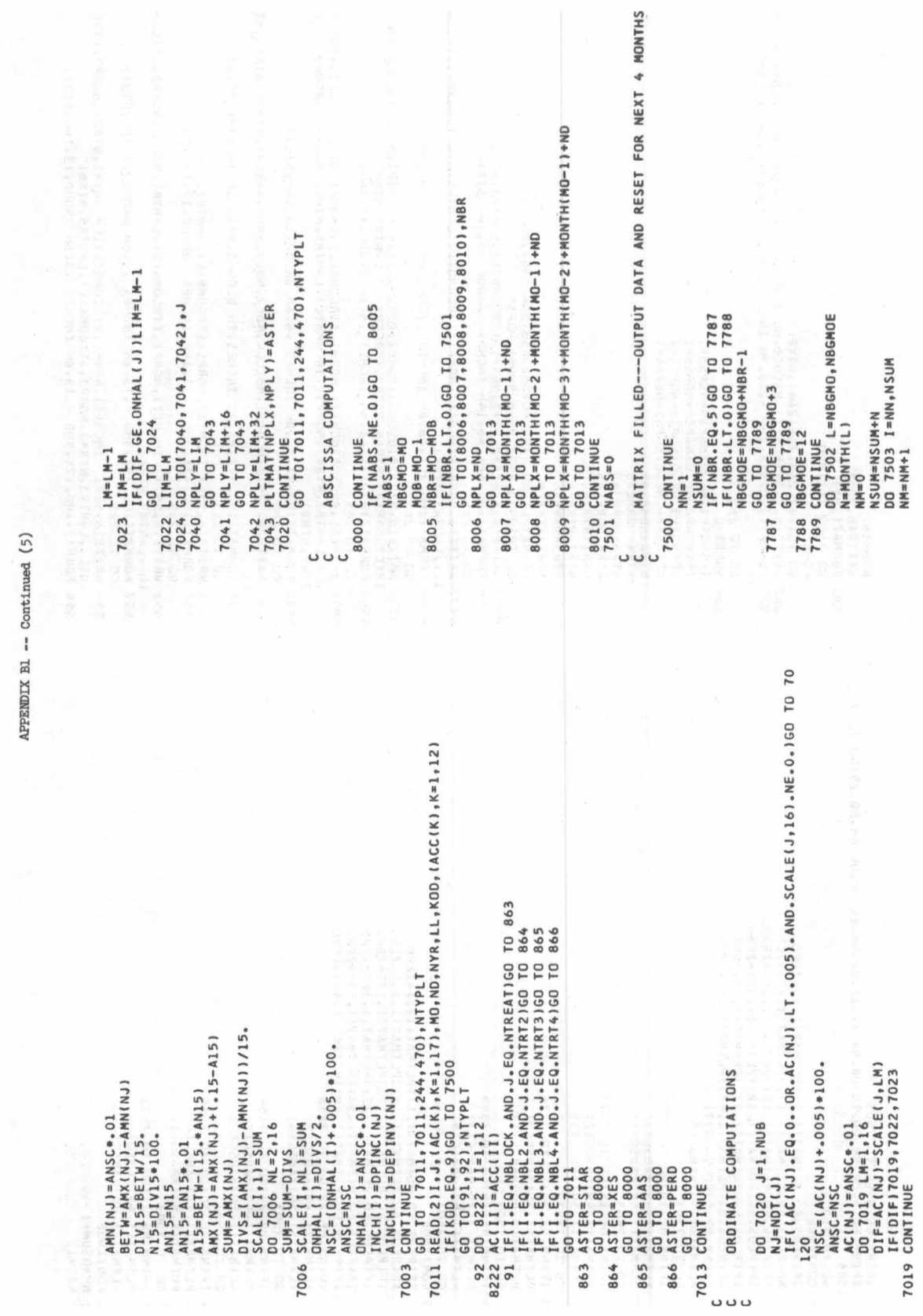




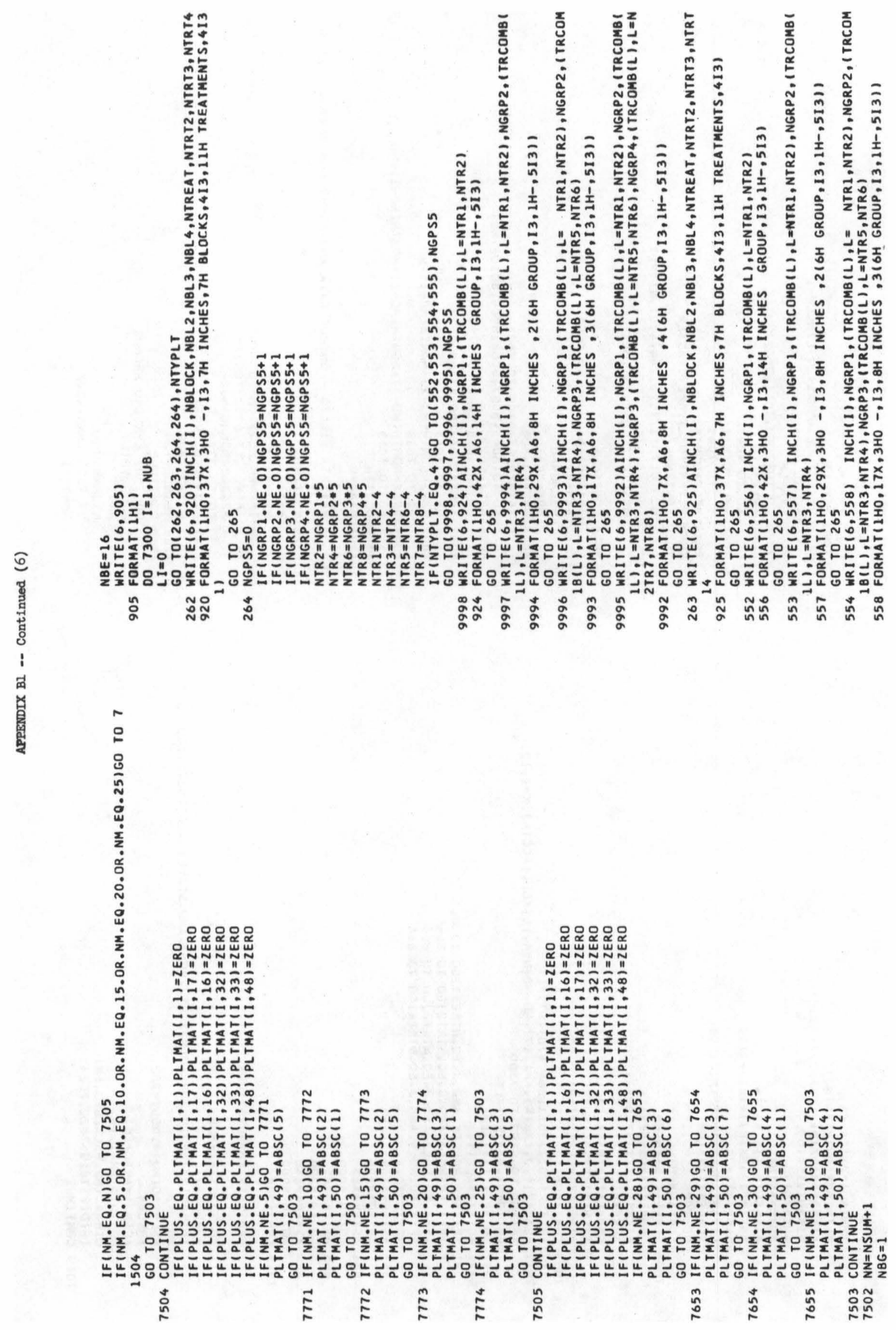




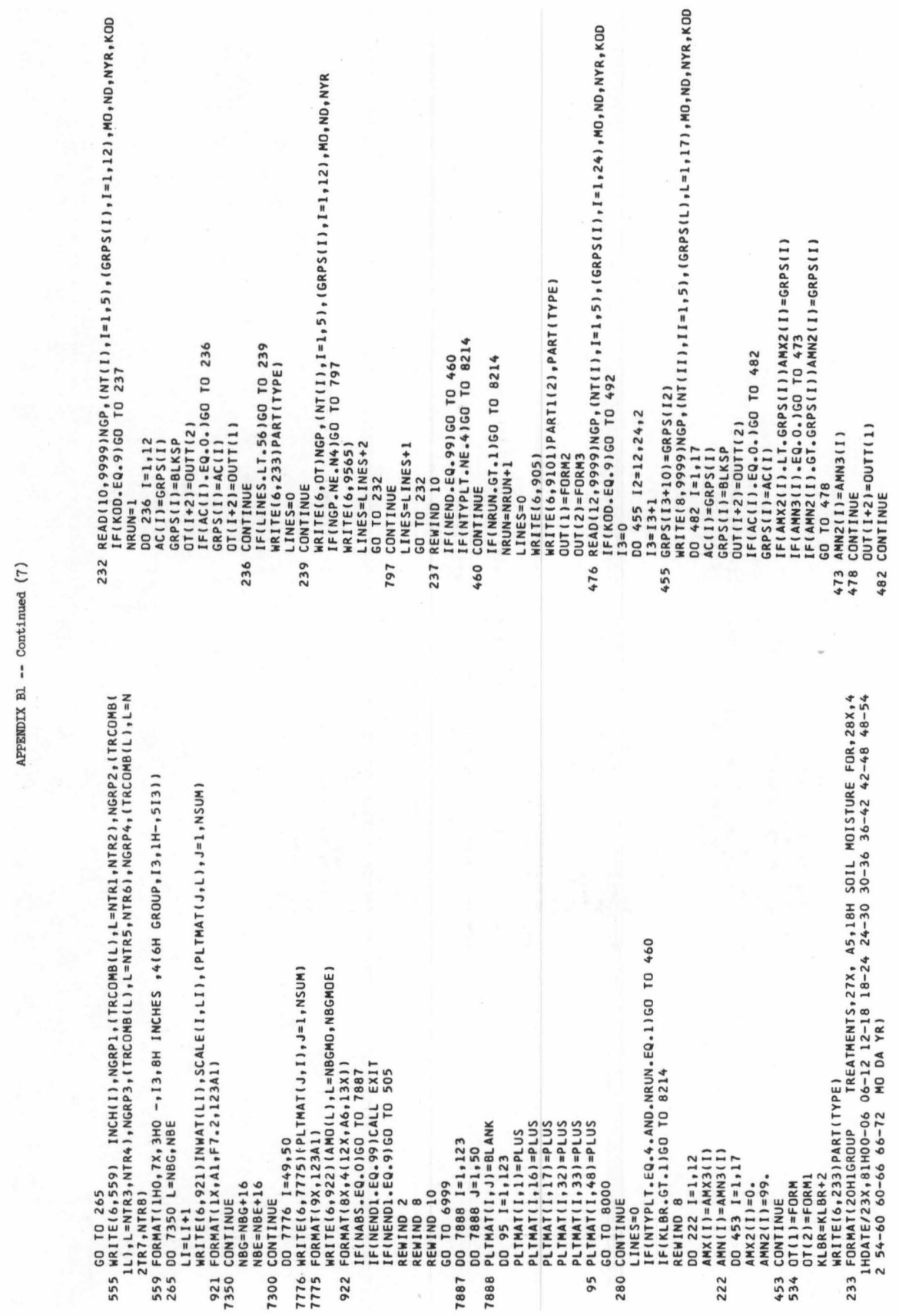




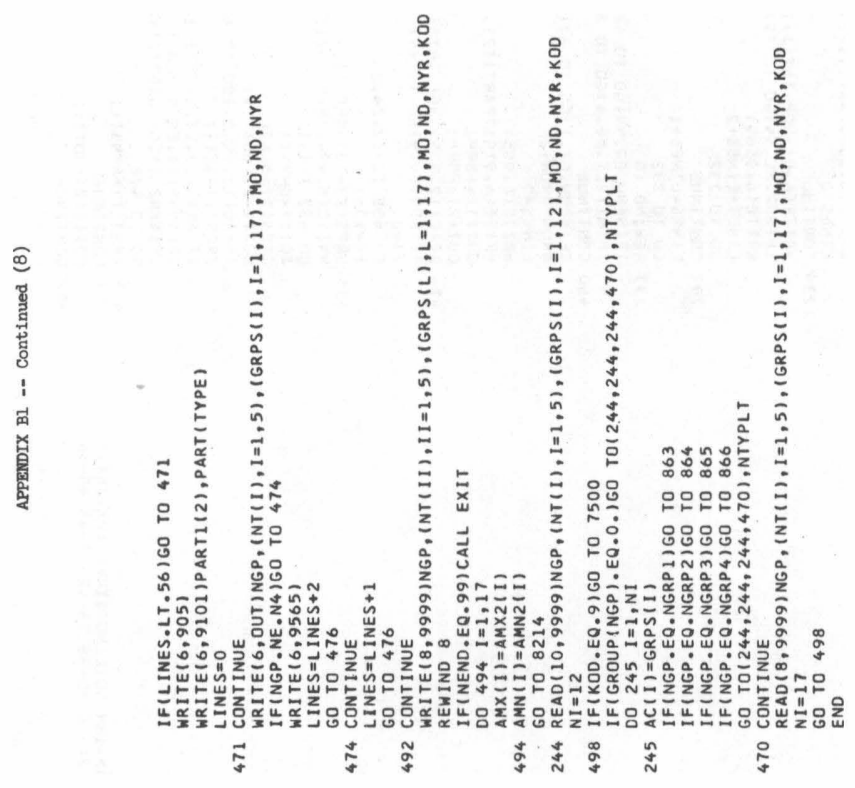




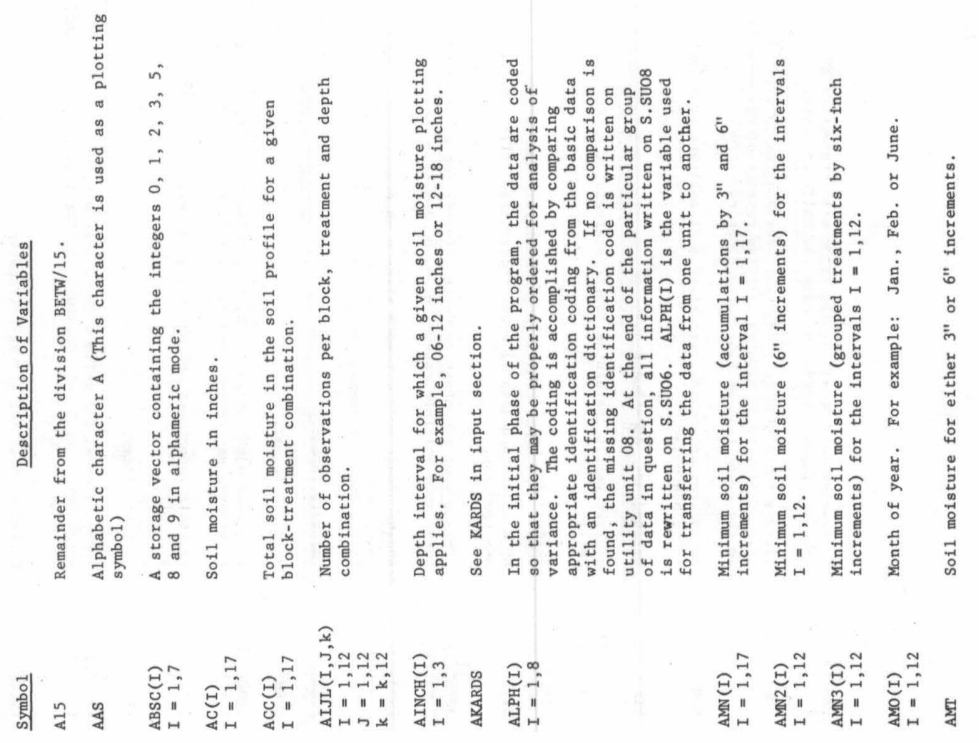




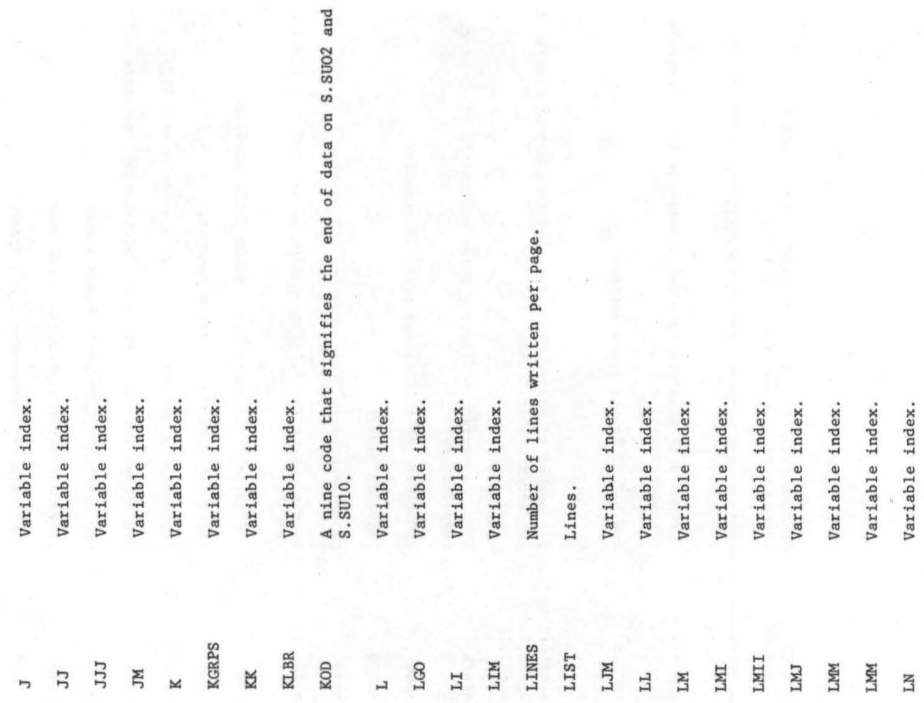

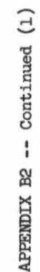

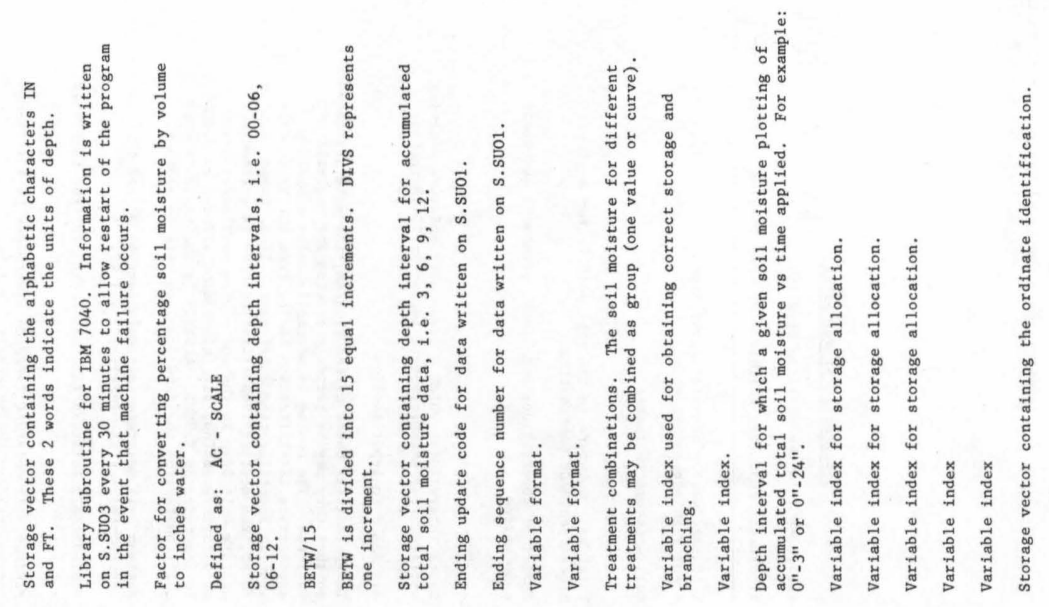

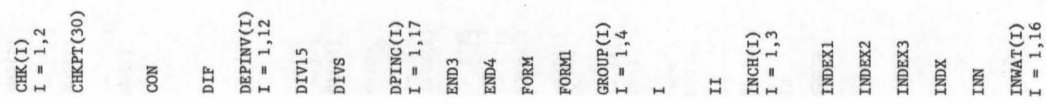




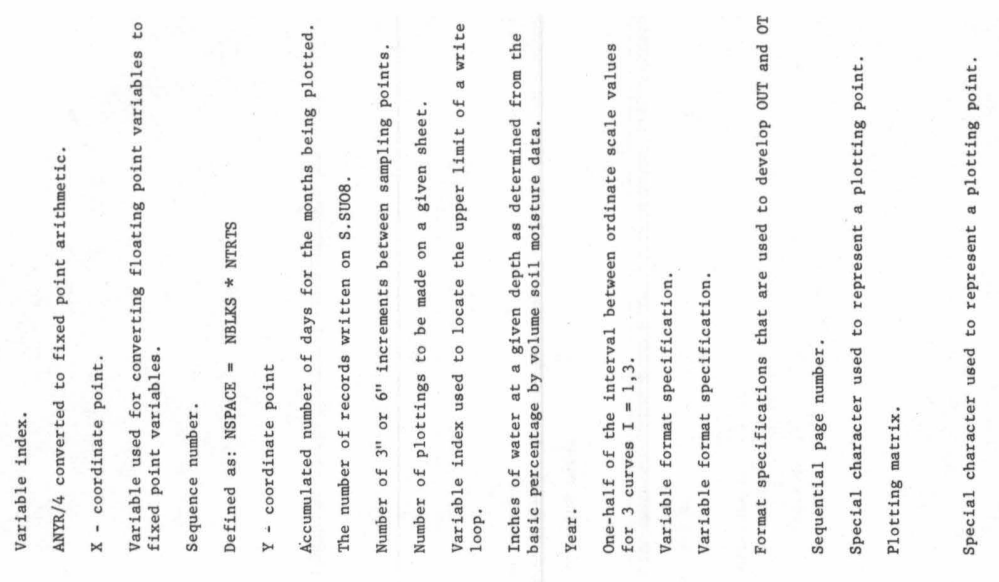

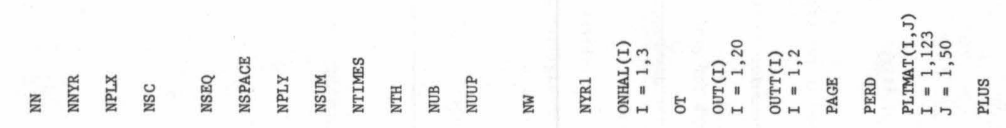
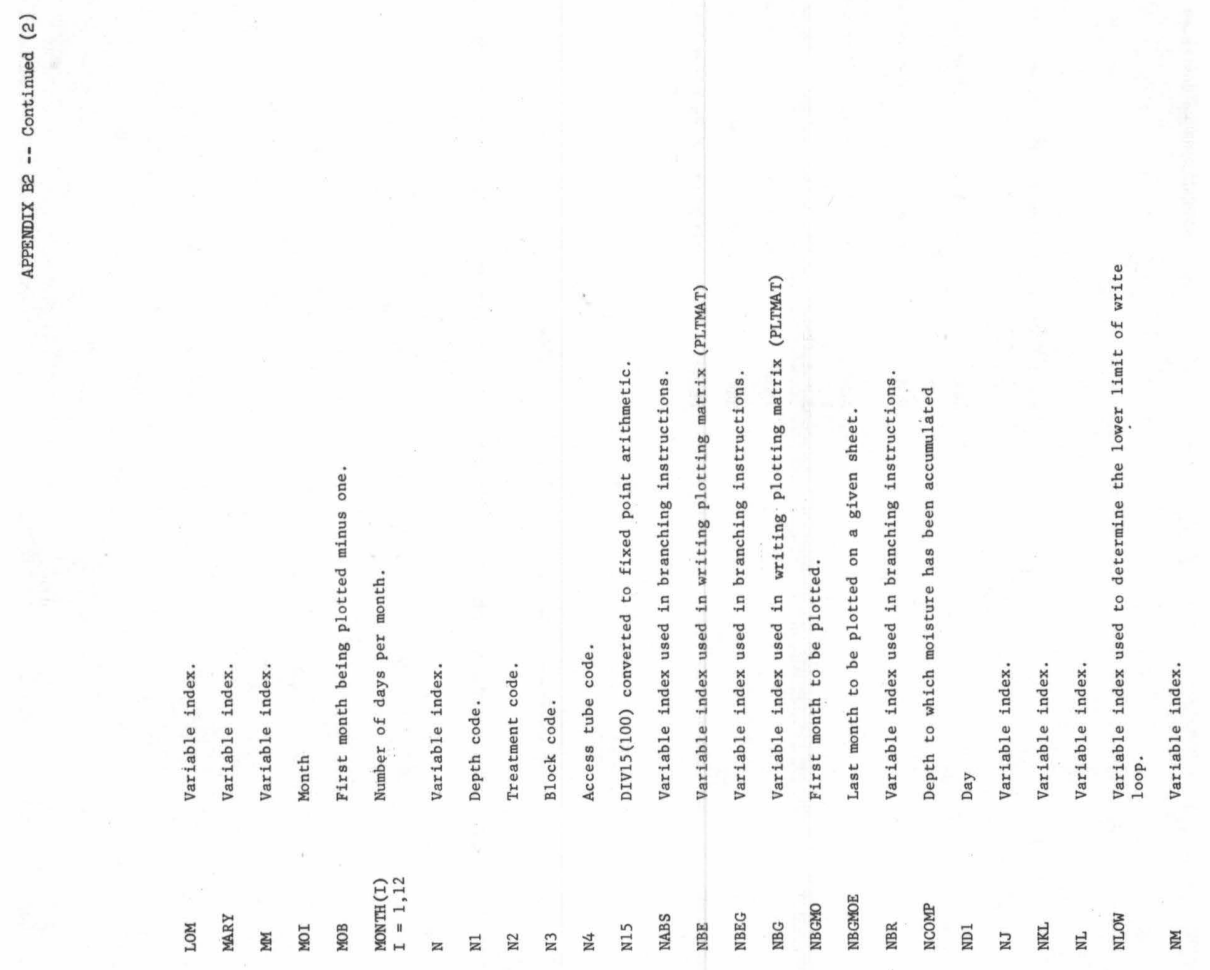


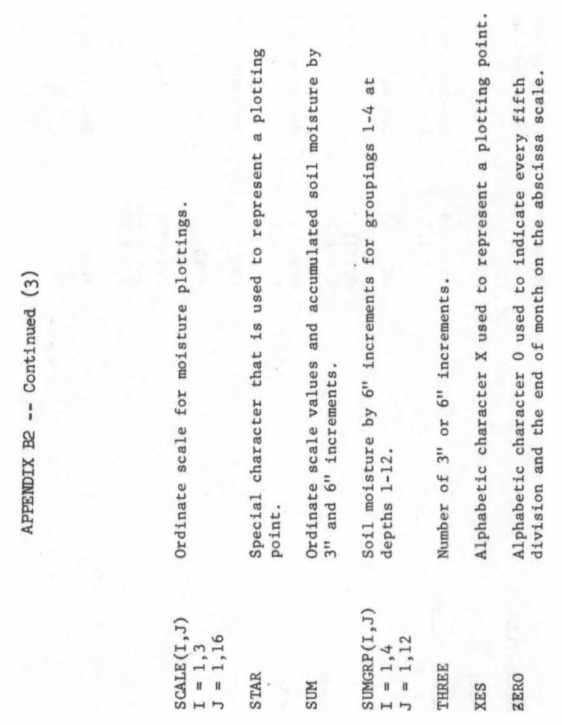


WATER RESOURCES RESEARCH CENTER

VIRGINIA POLYTECHNIC INSTITUTE BLACKSBURG, VIRGINIA 24061 